University of Louisville

ThinkIR: The University of Louisville's Institutional Repository

Electronic Theses and Dissertations

$6-2008$

\title{
Alternative piezoresistor designs for maximizing cantilever sensitivity.
}

Patrick Carl Fletcher

University of Louisville

Follow this and additional works at: https://ir.library.louisville.edu/etd

\section{Recommended Citation}

Fletcher, Patrick Carl, "Alternative piezoresistor designs for maximizing cantilever sensitivity." (2008). Electronic Theses and Dissertations. Paper 442.

https://doi.org/10.18297/etd/442

This Master's Thesis is brought to you for free and open access by ThinkIR: The University of Louisville's Institutional Repository. It has been accepted for inclusion in Electronic Theses and Dissertations by an authorized administrator of ThinkIR: The University of Louisville's Institutional Repository. This title appears here courtesy of the author, who has retained all other copyrights. For more information, please contact thinkir@louisville.edu. 
ALTERNATIVE PIEZORESISTOR DESIGNS

FOR MAXIMIZING CANTILEVER SENSITIVITY

\author{
By \\ Patrick Carl Fletcher \\ B.S., University of Louisville, 2006
}

\begin{abstract}
A Thesis
Submitted to the Faculty of the

University of Louisville

J.B. Speed School of Engineering as Partial Fulfillment of the Requirements

for the Professional Degree
\end{abstract}

\title{
MASTER OF ENGINEERING
}

Department of Mechanical Engineering

June 2008 



\section{ALTERNATIVE PIEZORESISTOR DESIGNS FOR MAXIMIZING CANTILEVER SENSITIVITY}

Submitted by:

Patrick Carl Fletcher

A Thesis Approved on

(Date)

by the Following Reading and Examination Committee:

Robert S. Keynton, Thesis Director

Roger D. Bradshaw

Bruce W. Alphenaar 


\section{ACKNOWLEDGMENTS}

This work is dedicated to my family for their steadfast love and continuing support of my academic goals. I would also like to thank my wife, Kimberly, and her family for their love, support, and encouragement in following my dreams.

I would like to express my sincere appreciation to my thesis advisor, Dr. Robert Keynton, for his unwavering guidance and support during my time spent working for him on my thesis. Dr. Keynton had a strong influence on my research and professional practices, having mentored me in the early stages of my career. Few men like Dr. Keynton excel on so many levels and exude such strong character and charisma. I would also like to thank Dr. Roger Bradshaw for his guidance on the analytical aspects of my research and for his advice in many other areas. Dr. Bradshaw was always willing to sit down and spend a few minutes talking about problems whenever I stumbled into a roadblock. In addition, I would like to thank Dr. Bruce Alphenaar for serving on my committee and for his guidance and support.

I would like to thank Thomas Roussel, Jr., Scott Cambron, Alex Isham, Scott Berry, Rathissh Dorairaj, Yang Xu, Matthew Anderson, Mark Crain, Joseph Lake, Ana Kieswetter, Prasanth Gopinath, and Joseph Williams for their help on various aspects of my research and for their instruction on different pieces of equipment. I would also like to express my appreciation to Sally Wren and Nancy Hansford for their support and encouragement. 


\begin{abstract}
ALTERNATIVE PIEZORESISTOR DESIGNS

FOR MAXIMIZING CANTILEVER SENSITIVITY
\end{abstract}

\title{
Patrick Carl Fletcher
}

July 11,2008

Over the last 15 years, researchers have explored the use of piezoresistive microcantilevers/resonators as gas sensors because of their relative ease in fabrication, low production cost, and their ability to detect changes in mass or surface stress with fairly good sensitivity. However, existing microcantilever designs rely on irreversible chemical reactions for detection and researchers have been unable to optimize symmetric geometries for increased sensitivity. Previous work by our group showed the capability of T-shaped piezoresistive cantilevers to detect gas composition using a nonreaction-based method - viscous damping. However, this geometry yielded only small changes in resistance. Recently, computational studies performed by our group indicated that optimizing the geometry of the base piezoresistor increases device sensitivity up to 700 times. Thus, the focus of this work is to improve the sensitivity of nonreaction-based piezoresistive microcantilevers by incorporating asymmetric piezoresistive sensing elements in a new array design.

A three-mask fabrication process was performed using a 4" silicon-on-insulator wafer. Gold bond pads and leads were patterned using two optical lithography masks, gold sputtering, and acetone lift-off techniques. The cantilevers were patterned with 
electron-beam lithography and a dry etch masking layer was then deposited via electronbeam evaporation of iron. Subsequently, the silicon device layer was deep reactive ion etched (DRIE) to create the vertical sidewalls and the sacrificial silicon dioxide layer was removed with a buffered oxide etch, completely releasing the cantilever structures.

Finally, the device was cleaned and dried with critical point drying to prevent stiction of the devices to the substrate. For the resonance experiments, the cantilevers were driven electrostatically by applying an AC bias, $10 \mathrm{~V}_{\mathrm{pp}}$, to the gate electrode. A DC bias of $10 \mathrm{~V}$ was placed across the piezoresistor in series with a $14 \mathrm{k} \Omega$ resistor. The drive frequency $(0$ $-80 \mathrm{kHz}$ ) was swept until the cantilever resonated at its natural frequency, which occurred when the output of the lock-in amplifier reached its maximum. These devices have been actuated to resonance under vacuum and their resonant frequencies and Qfactors measured.

The first mode of resonance for the asymmetric cantilevers was found to range between $40 \mathrm{kHz}$ and $63 \mathrm{kHz}$, depending on the piezoresistor geometry and length of the cantilever beam. The redesigned piezoresistive microcantilevers tested yielded static and dynamic sensitivities ranging from 1-6 $\Omega / \mu \mathrm{m}$ and $2-17 \Omega / \mu \mathrm{m}$ displacement, respectively, which are $40-730$ times more sensitive than the best symmetric design previously reported by our group. Furthermore, the Q-factors ranged between 1700 and 4200, typical values for MEMS microcantilevers. 


\section{TABLE OF CONTENTS}

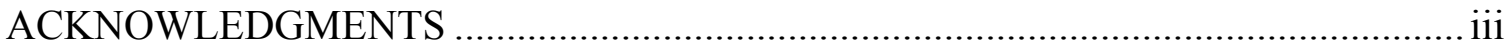

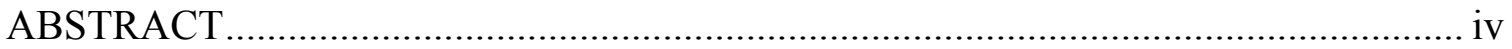

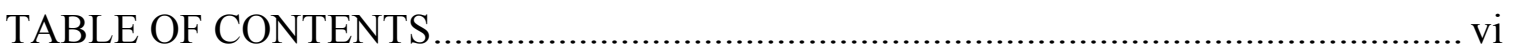

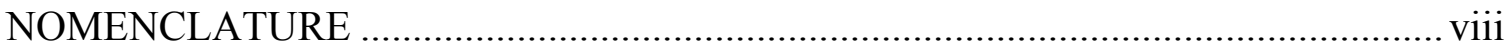

LIST OF TABLES

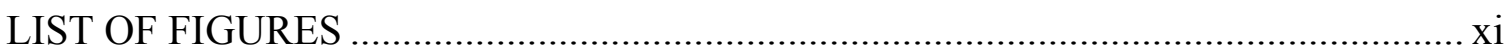

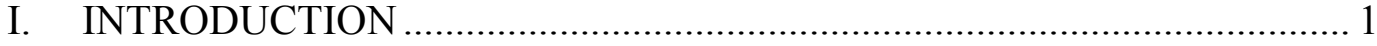

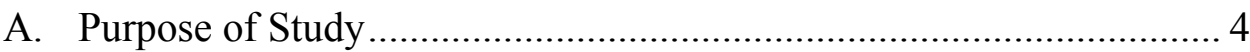

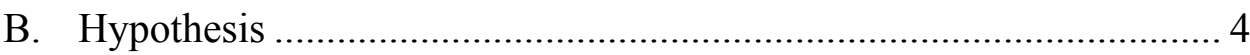

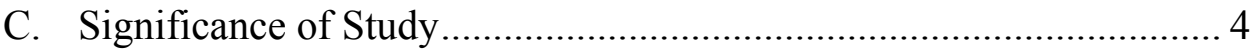

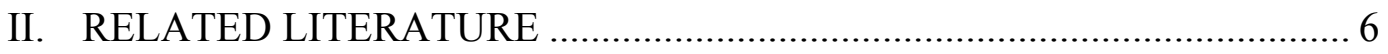

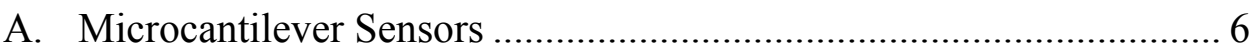

B. Microcantilevers for Physical, Biological, and Chemical Sensing...... 7

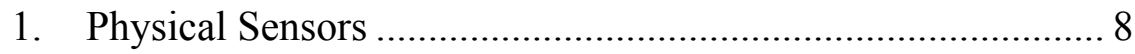

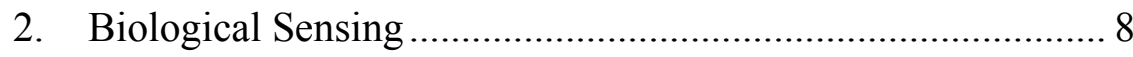

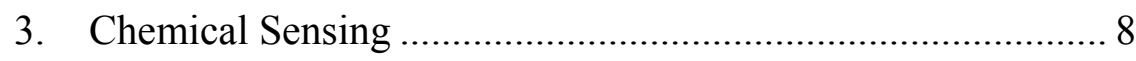

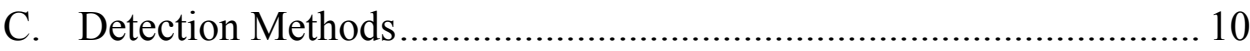

1. Optical Lever Method.......................................................... 10

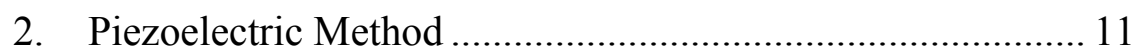

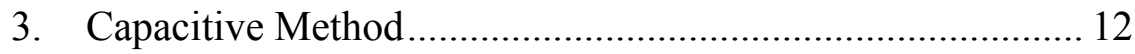

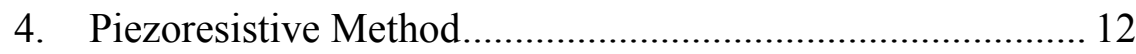

D. Piezoresistive Microcantilever Sensors …………............................ 13

E. Reaction Versus Non-Reaction Microcantilever Sensors.................... 14

1. Viscous Damping of a Resonating Microcantilever ............... 15

F. Previous Work With Symmetric Microcantilevers.............................. 16

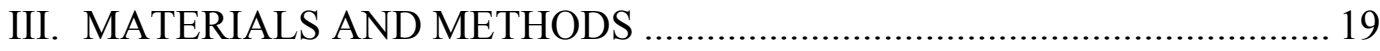

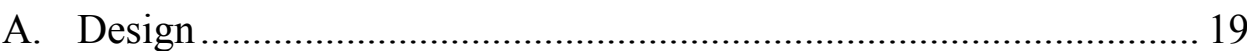

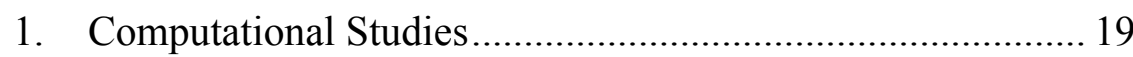

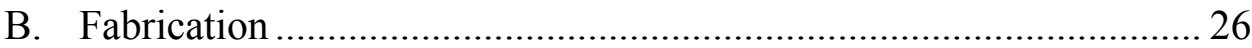

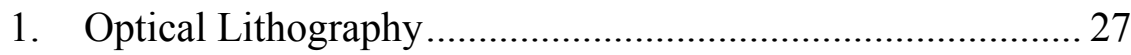

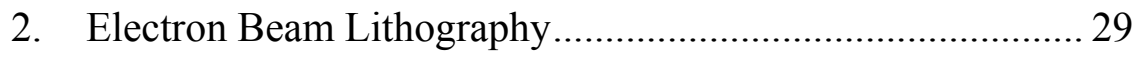

3. Dry Etching Process .............................................................. 32

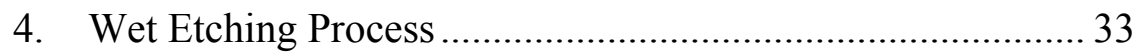

5. Critical Point Drying Process ............................................. 34

C. Piezoresistive Detector Characterization Studies ............................... 35

1. Determination of Static Piezoresistor Resistance ................... 36 
2. Microcantilever Metrology .................................................... 37

D. Resonator and Sensitivity Detection Experiments ............................. 38

1. Resonator Actuation Experiments ......................................... 38

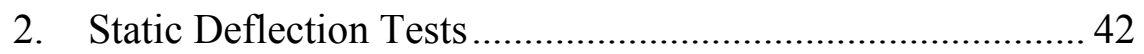

3. Dynamic Deflection Tests .................................................... 44

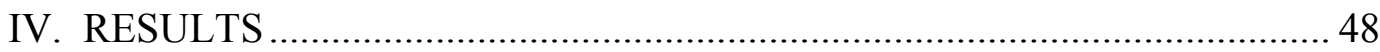

A. Determination of Static Piezoresistor Resistance ............................... 48

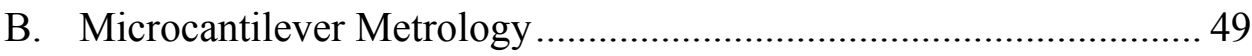

C. Resonator Actuation Experiments ……………………………........... 54



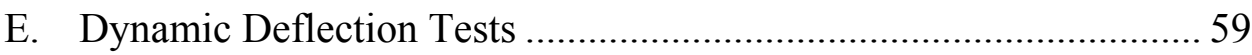

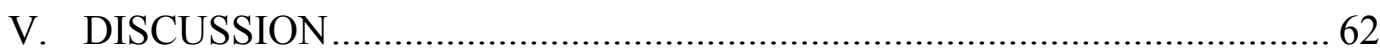

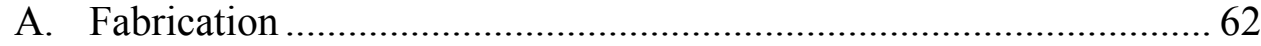

B. Determination of Static Piezoresistor Resistance .................................67

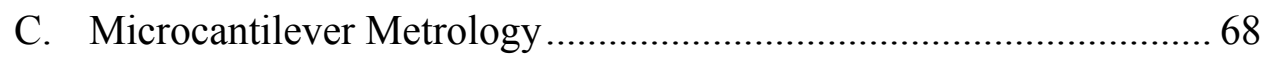

D. Resonator Actuation Experiments ……………………………......... 70

E. Static Deflection Tests.................................................................. 71



VI. CONCLUSIONS AND RECOMMENDATIONS ……………….................. 76

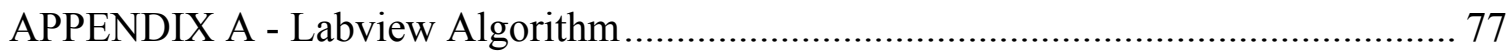

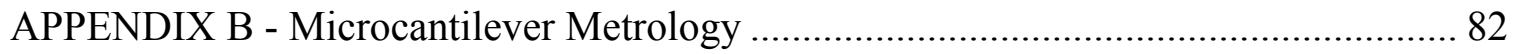

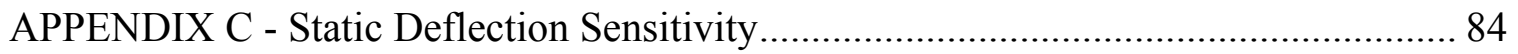

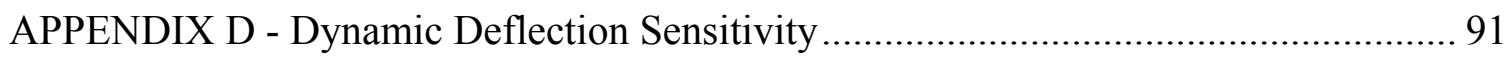

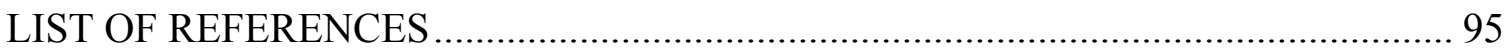

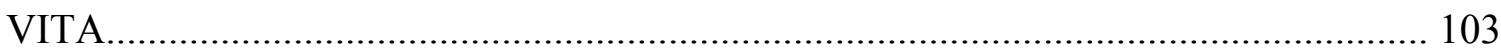




\section{NOMENCLATURE}

$\begin{array}{ll}{ }^{\circ} \mathrm{C} & =\text { degrees Celsius } \\ \mu & =\text { dynamic viscosity of gas } \\ \mu \mathrm{A} & =\text { microamp } \\ \mu \mathrm{m} & =\text { micrometer } \\ \mathrm{A} & =\text { angstrom } \\ \mathrm{AC} & =\text { alternating current } \\ \mathrm{AFM} & =\text { atomic force microscope } \\ \mathrm{BOE} & =\text { buffered-oxide etch } \\ \mathrm{CL} & =\text { cantilever length } \\ \mathrm{cm} & =\text { centimeter } \\ \mathrm{CMOS} & =\text { complementary metal-oxide-semiconductor } \\ \mathrm{DC} & =\text { direct current } \\ \mathrm{deg} & =\text { degree } \\ \mathrm{DI} & =\text { de-ionized } \\ \mathrm{DRIE} & =\text { deep-reactive ion etch } \\ \mathrm{E} & =\text { Young's modulus of cantilever material } \\ \mathrm{f} & =\text { resonance frequency in vacuum } \\ \mathrm{FEA} & =\text { finite element analysis } \\ \mathrm{GHz} & =\text { gigahertz } \\ \mathrm{GPIB} & =\text { general purpose interface bus } \\ \mathrm{Hz} & =\text { hertz } \\ \mathrm{IPA} & =\text { isopropyl alcohol } \\ \mathrm{k} & =\text { cantilever spring constant } \\ \mathrm{K} & =\text { Kelvin } \\ \mathrm{kHz} & =\text { kilohertz } \\ \mathrm{kV} & =\text { kilovolts } \\ \mathrm{k} \Omega & =\text { kilohm } \\ \mathrm{L} & =\text { cantilever beam length } \\ \mathrm{LL} & =\text { piezoresistor leg length } \\ \mathrm{LOR} 3 \mathrm{~A} & =\text { liftoff resist } 3 \mathrm{~A} \\ \mathrm{LS} & =\text { piezoresistor leg separation } \\ \mathrm{m} & =\text { mass of cantilever } \\ \mathrm{M} & =\text { molar mass of gas } \\ \mathrm{m} \mathrm{b} & =\text { mass of the cantilever } \\ \mathrm{MEMS} & =\text { micro-electro-mechanical systems } \\ \mathrm{MHz} & =\text { megahertz } \\ & \end{array}$




$$
\begin{array}{ll}
\text { MIBK } & =\text { methyl isobutyl ketone } \\
\mathrm{nC} & =\text { nanocoulomb } \\
\mathrm{NEMS} & =\text { nano-electrical-mechanical systems } \\
\mathrm{nm} & =\text { nanometer } \\
\mathrm{NPGS} & =\text { nanometer pattern generation system } \\
\mathrm{P} & =\text { pressure } \\
\mathrm{pH} & =\text { acidity or alkalinity of a solution } \\
\mathrm{PL} & =\text { piezoresistor length } \\
\mathrm{PMMA} & =\text { polymethyl methacrylate } \\
\mathrm{PSD} & =\text { position-sensitive detector } \\
\mathrm{PW} & =\text { piezoresistor width } \\
\mathrm{Q}-\text { factor } & =\text { quality factor } \\
\mathrm{R} & =\text { radius of sphere } \\
\mathrm{R}_{0} & =8.314 \mathrm{~J} / \text { (mol } \cdot \mathrm{K}) \\
\mathrm{R}_{0} & =\text { resistance at non-resonance } \\
\mathrm{R}_{0} & =\text { resistance at zero deflection } \\
\mathrm{R}_{1} & =\text { maximum resistance at deflection } \Delta \mathrm{x} \\
\mathrm{R}_{1} & =\text { resistance at resonance } \\
\mathrm{RF} & =\text { radio frequency } \\
\mathrm{Rpm} & =\text { rotations per minute } \\
\mathrm{sccm} & =\text { standard cubic centimeters per minute } \\
\mathrm{SEM} & =\text { scanning electron microscope } \\
\mathrm{SOI} & =\text { silicon-on-insulator } \\
\mathrm{T} & =\text { absolute temperature } \\
\mathrm{t} & =\text { cantilever thickness } \\
\mathrm{UV} & =\text { ultra-violet } \\
\mathrm{V} & =\text { total tip displacement } \\
\mathrm{V} & =\text { Volts } \\
\mathrm{VCSEL} & =\text { vertical-cavity surface-emitting laser } \\
\mathrm{W} & =\text { Watt } \\
\mathrm{W} & =\text { width of cantilever } \\
\mathrm{W} & =\text { width of cantilever } \\
\Delta \mathrm{f} & =\text { shift of the resonance frequency } \\
\Delta \mathrm{x} & =\text { deflection of cantilever tip } \\
\Omega & =\text { Ohm } \\
\omega_{\mathrm{o}} & =\text { cantilever resonant frequency } \\
&
\end{array}
$$




\section{LIST OF TABLES}

Table I - Asymmetric Microcantilever Geometry Designs.............................................. 25

Table II - Lock-In Amplifier Gain at Selected Sensitivity Values ................................. 47

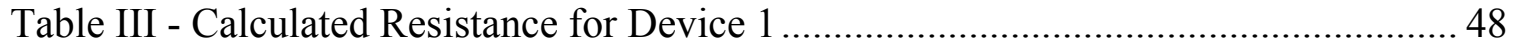

Table IV - Percent Error for Geometry Parameters and Overall Error........................... 54 


\section{LIST OF FIGURES}

Figure 1 - Schematic of "Optical Lever" Detection Method (Lavrik, 2004)..................... 11

Figure 2 - Laterally Vibrating Silicon Microcantilever with Symmetric, T-Shaped

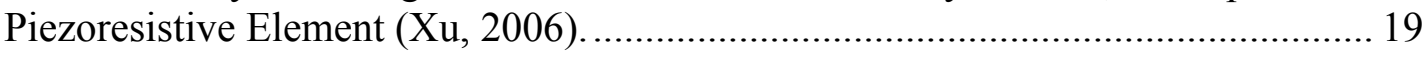

Figure 3 - Free-Body Diagram of T-Shaped Piezoresistive Element (Bradshaw, et al.

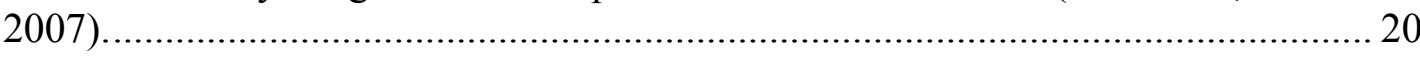

Figure 4 - Free-Body Diagram of Asymmetric Piezoresistive Element (Bradshaw, et al.

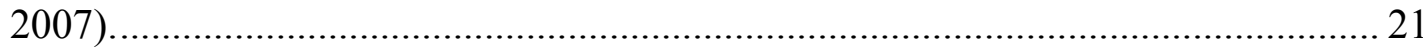

Figure 5 - Symmetric Microcantilever Geometry ........................................................... 22

Figure 6 - Normalized Resistance For T-Shaped Model in $<110>$ Orientation and $1.1 \mu \mathrm{m}$

Thick Piezoresistor (Bradshaw, et al. 2007). ............................................................ 23

Figure 7 - Normalized Resistance for Asymmetric Model in $<110>$ Orientation and 1.1

$\mu \mathrm{m}$ Thick Piezoresistor (Bradshaw, et al. 2007)..................................................... 23

Figure 8 - Asymmetric Microcantilever Geometry................................................... 24

Figure 9 - Device Layout with 10 Microcantilever Beams in an Array. ……………..... 26

Figure 10 - Schematic for Fabrication Process (Xu, 2006)........................................... 27

Figure 11 - Design Layout of the Optical Lithography Mask Files. Mask 1 was used first

to pattern the electrical leads, followed by mask 2 to pattern the bond pads........... 28

Figure 12 - Example of Cantilever Pattern for E-Beam Writing ....................................... 31

Figure 13 - Probe Station Used to Measure Resistance Across Piezoresistors.................. 37

Figure 14 - Typical Graph of Voltage Versus Current Across a Piezoresistor.................. 37

Figure 15 - Microcantilever Geometry Measurements................................................. 38

Figure 16 - Electrode Layout for Microcantilever Device............................................... 39

Figure 17 - Schematic of General Instrumentation Setup. ( - BNC Cable, (- - - )

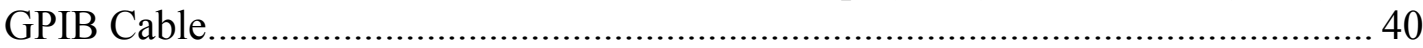

Figure 18 - Instruments Used in the Experimental Setup.............................................. 40

Figure 19 - LabVIEW VI Entitled Sweep_Freq2.Vi Used To Sweep The Resonant

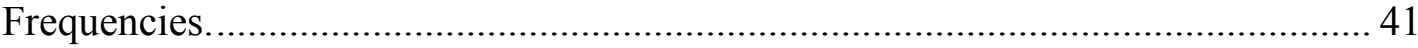

Figure 20 - A Beam5 Microcantilever Resonating In The Probe Station........................ 42

Figure 21 - Tungsten Needle Statically Actuating a Microcantilever in an SEM. ........... 43

Figure 22 - Nanomanipulators and Microcantilever Device in an SEM. ......................... 44

Figure 23 - Custom-Made Prototyping Circuit Board for Imaging Microcantilever Array

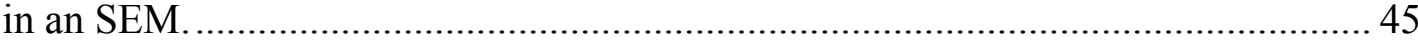

Figure 24 - Schematic Drawing of the Resonating Cantilever Deflection Measurement. 45

Figure 25 - Schematic of Voltage Divider Circuitry. ....................................................... 47

Figure 26 - Mean Leg Length and Standard Deviation for Measured Microcantilevers. . 50

Figure 27 - Mean Leg Separation and Standard Deviation for Measured Microcantilevers. 
Figure 28 - Mean Beam Length and Standard Deviation for Measured Microcantilevers.

Figure 29 - Mean Beam Width and Standard Deviation for Measured Microcantilevers.51

Figure 30 - Percent Error in Leg Length Parameter Fabrication. ................................... 52

Figure 31 - Percent Error in Leg Separation Parameter Fabrication. .............................. 52

Figure 32 - Percent Error in Beam Length Parameter Fabrication. .................................. 53

Figure 33 - Percent Error in Beam Width Parameter Fabrication. ................................... 53

Figure 34 - Measured and (Theoretical/2) Resonant Frequencies of Cantilever Devices. 55

Figure 35 - Graph of Resistance Versus Deflection for Device 7, Beam 6 .................... 57

Figure 36 - Graph of Resistance Versus Deflection for Device 1, Beam 2.................... 57

Figure 37 - Static Sensitivity of Cantilever Devices...................................................... 58

Figure 38 - Mean Static Sensitivity of Cantilever Devices, Beam Length Constant........ 58

Figure 39 - Dynamic Sensitivity Of Cantilever Devices. ................................................ 60

Figure 40 - Mean Dynamic Sensitivity of Cantilever Devices, Beam Length Constant. . 60

Figure 41 - Dynamic and Static Sensitivity Of Cantilever Devices. ................................. 61

Figure 42 - E-Beam Exposure of Ten Microcantilevers Simultaneously After

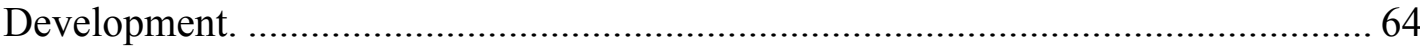

Figure 43 - Alignment Artifacts From E-Beam Writing. ................................................ 64

Figure 44 - Overetching Microcantilever in DRIE; Iron Masking Layer Peeling Off. .... 65

Figure 45 - SEM Micrograph of a Microcantilever That Was Removed From the Liquid

BOE and Rinsed in DI Water and IPA Before Critical Point Drying........................ 66

Figure 46 - SEM Micrograph of Microcantilever After Brief (Under 1 Second) Dunk in

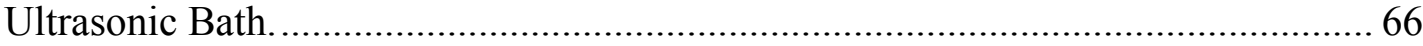




\section{INTRODUCTION}

Micro-electrical-mechanical systems (MEMS) have been generating increasing research interest in the past two decades, which has resulted in an exponential growth of commercially-available MEMS devices (Salzberg, 2002). MEMS and Nano-electricalmechanical systems (NEMS) have found widespread applications in the fields of sensors, actuators, and other microsystems (Judy, 2001; Blencowe, 2005; Craighead, 2000; Roukes, 2001). MEMS devices typically operate at the micron scale and are characterized as very small machines utilizing the mechanical and electrical properties of silicon and other semiconductor materials. MEMS devices are typically fabricated using micromachining techniques classically reserved for semiconductor fabrication (Judy, 2001).

Micro-mechanical resonators constitute a large portion of MEMS and NEMS devices and are making significant contributions to the sensing field (Lavrik, 2004; Porter, 2001; Ziegler, 2004). Resonators operate on the principle that a physical, chemical, or biological stimulus will change the mechanical characteristics of the resonator, producing a change in frequency, amplitude, and/or quality-factor (Q-factor) output signal. In this way, the resonator acts as a transducer by converting the stimulus input into an electrical output signal. The resulting changes can be measured using electronic, optical, or other sensing means (Carr, 1999; Scuor, 2006).

Micro/Nanofabricated structures create ideal platforms for high sensitivity resonators because of their ability to generate high resonance frequencies and high Q-factors, due to their small size and mass (Yao, 2000). Thus, micro and nano resonators have been used 
for a variety of applications such as gas detection (Chopra, 2004; Thiele, 2003; Xu, 2006; Zribi, 2005), mass (Gupta, 2004; Ekinci, 2003; Abedinov, 2001), heat flux (Abedinov, 2001; Volklein, 1999; Wang, 2005), force (Kenny, 2001; Mei, 2000; Chui, 1998), surface stress (Datskos, 2001; Preissig, 2001; Muller, 2001), and charge (Riehl, 2003) sensors.

In regards to gas sensing, microresonators have primarily measured changes in mass or surface stress through a chemical interaction between the gas and the resonator material/surface, which causes a measurable shift in the resonance frequency (Zribi, 2005). The resonant frequency of the microresonator can be measured through electronic circuitry using the piezoelectric effect (Wang, 2003) or the piezoresistive effect (Partridge, 2000), and can also be measured optically using a laser focused on the vibrating structure (Gupta, 2004).

Microcantilevers, in particular, are a special type of microresonator well-suited for gas detection because of the relatively simple fabrication methods required, suitability for arrangement in an array, and relatively large displacement compared to other microresonators (Ziegler, 2004). Microcantilevers have been utilized in many other applications besides gas sensing such as atomic-force microscopes (AFM) (Albrecht, 1990), accelerometers (Kim, 1995), etc. The majority of microcantilevers fabricated, to date, use a symmetric geometry for the base of the cantilever beam (Su, 2003; Lavrik, 2004; Xu, 2006; Lee, 2003) and a piezoresistive or piezoelectric sensing element (Partridge, 2000; Wang, 2003). For example, previous work by our group involved the fabrication of a T-shaped piezoresistive sensing element at the base of the cantilever for performing gas composition analysis (Xu, 2006). The T-shaped geometry yielded small, 
but measurable, changes in resistance while the cantilever resonated, due to bending stresses in the support structure of the cantilever as the tip was displaced. However, the beneficial axial stresses largely canceled due to the symmetric geometry of the cantilever base; thereby, decreasing the sensitivity of the sensor.

These measurements are carried out by measuring the stress in the 'piezo' element, which when increased should enhance the sensitivity of the cantilever. Currently, the stress in the piezo element can be increased by: 1) lengthening the cantilever; 2) increasing the stress concentration with sharper corners; and/or, 3) increasing the deflection of the resonating cantilever. However, there are several limitations to these methods. Specifically, increasing the length of the cantilever adds mass to the beam, which decreases the natural frequency and leads to a lower Q-factor. Regarding stress concentration, it is difficult to consistently produce sharp features with conventional micromachining techniques since the minimum feature size that can be produced by traditional lithographic techniques is $1 \mu \mathrm{m}$. Furthermore, increasing the deflection of the cantilever requires a larger $\mathrm{AC}$ drive voltage, which means more power consumption and more equipment.

An alternative method for increasing the stress in the 'piezo' element must be developed. An unutilized alternative is to vary the geometry of the 'piezo' element, creating more favorable coupling stresses in the element. Thus, the objective of this work is to demonstrate that altering the geometry of the piezoresistive element in a resonating microcantilever will improve the cantilever sensitivity. 


\section{A. Purpose of Study}

The purpose of this study was to fabricate and test the sensitivity of silicon microcantilever arrays with varying asymmetric piezoresistive sensing element geometries.

B. Hypothesis

The hypothesis of this study is that piezoresistive sensing microcantilevers with asymmetric geometries will have greater sensitivity than a symmetric, T-shaped design.

C. Significance of Study

A common problem with existing microcantilever based gas sensors is the need for chemically-reactive coatings on the cantilever for absorbing or desorbing the desired analyte. This technique has limitations:

- The coating must be designed with a suitable reaction mechanism;

- The coating must be precisely placed without damaging the cantilever;

- The types of gases that can be detected are limited;

- Unknown or inert gases cannot be detected; and,

- The detection process is usually irreversible.

Recently, Xu, et al. (2006) explored new alternative cantilever designs, which are non-reaction-based for gas detection. This cantilever utilizes the damping effect of gases to distinguish between gases with different molar masses. The major advantage of this technique is that it does not rely on a chemical reaction. However, the T-shaped design of the microcantilever sensing element developed by $\mathrm{Xu}$ yielded low sensitivity values.

Xu's best device sensitivity was $0.0294 \Omega / \mu \mathrm{m}$ and her T-9 resonators had an average relative resistance change of $2.7 \times 10^{-6}$ for a $1 \mu \mathrm{m}$ deflection of the free end of the 
cantilever. This $10^{-6}$ relative resistance change approaches the detection limit of the Wheatstone bridge configuration used to measure the change in piezoresistance. Lang et al. (2005) identified microcantilevers as very capable chemical and biological sensors, but highlighted the need for optimized device design to further improve sensitivity. In the future if these microcantilevers are to be used in a handheld package then the microcantilever sensitivity, or the change in piezoresistance during resonance, must be improved so that conventional integrated circuit packages can be used in place of labgrade equipment, such as a lock-in amplifier, to detect changes in microcantilever resonance. New piezoresistor element designs must be formulated to enhance device sensitivity. An asymmetric piezoresistor element which increases microcantilever sensitivity is the first step in the improvement of these novel MEMS sensing devices. 


\section{RELATED LITERATURE}

Current research and production of MEMS devices mainly focuses on actuationbased sensors for physical (Morante, 1996; Agoston, 2005; Mamin, 2001; Stowe, 1997), chemical (Lange, 1999; Thundat, 1995; Ji, 2001; Butt, 1995), and biological (Ilic, 2000; Baselt, 1996; Grogan, 2002; Antonik, 1997) sensing. In the last two decades, it has become possible to produce inexpensive MEMS sensors through batch silicon micromachining techniques developed for the integrated circuit industry. MEMS microactuators typically consist of beams (Yasuda, 1997; Pan, 1997) and diaphragms (Carlen, 1999; Hirata, 1996), though microactuated beams have demonstrated the highest detection sensitivity, repeatability, and reproducibility (Li, 2003; Stowe, 1997; Ilic, 2000).

\section{A. Microcantilever Sensors}

The microcantilever is the most common type of MEMS microactuator, which is characterized by a suspended single-clamped beam that acts as the sensing element. The microcantilever sensor works by detecting changes in mechanical stress or resonance response of the beam, which statically or dynamically indicates changes in deflection or damping of the cantilever, respectively. Commercial cantilevers are typically made of silicon, silicon nitride, or silicon oxide (Ziegler, 2004).

Microcantilevers were initially used in contact with other surfaces and were characterized by low spring constants with high sensitivity to applied forces and high resonance frequencies for faster response times (Ziegler, 2004). Resonating cantilevers have shown advantages in detecting minute quantities of external stimuli due to their 
naturally high resonant frequencies and high Q-factors (Yao, 2000). The evolution of the microcantilever has led to sensors that no longer bring surfaces into contact with the microcantilever. Instead, the microcantilevers act as miniature transducers based on fundamental principles of physics like the bimetallic effect (Chu, 1993), mechanical stress (Bargatin, 2005), or the harmonic oscillator (Lee, 1996).

There are typically three ways that microcantilevers are used to transduce an input stimulus into a measureable output: measure mass loading from a frequency change, monitor temperature change from bimetallic cantilever deformation, or sense surface stress on one side of the cantilever from cantilever deformation (Ziegler, 2004). Arranging different microcantilevers into an array has enormous potential for improving the reliability, sensitivity, and selectivity of microcantilever-based sensors.

B. Microcantilevers for Physical, Biological, and Chemical Sensing

Lang et al. (2005) have investigated the possible applications and uses for microcantilevers over many years. Their investigation has found that an array of microcantilevers can function in many capacities, including as an artificial nose for the detection of vapors and as a biological detector capable of detecting specific DNA sequences. The cantilever coatings can be applied by a cost-effective ink-jet spotting device, or by insertion into solution-filled glass capillaries. They concluded that a cantilever sensor array is highly capable of detecting physisorption and chemisorption processes, as well as determining material-specific properties such as enthalpy changes during phase transitions. However, Lang and his colleagues indicate that the challenge in cantilever sensor array technology lies in optimizing the cantilever sensors to improve their sensitivity. 


\section{Physical Sensors}

Microcantilever beams can be extremely sensitive to physical stimuli. Stowe et al. (1997) developed a microcantilever capable of detections attonewtons at $4.8 \mathrm{~K}$ in a vacuum. Microcantilever beam sensors have also been used to measure the viscosity of complex organic liquids (Agoston, 2005), low frequency acceleration (Morante, 1996), gas flow velocity (Su, 2002), and temperature (Thundat, 1995).

\section{Biological Sensing}

Biosensing with cantilevers requires an understanding of the complex biochemical processes taking place on the cantilever, and therefore is more difficult than nonbiological microcantilever sensing. Cells can be cultured on the surfaces of cantilevers and these cell/cantilever platforms can detect the response of cells to external stimuli (Antonik, 1997). Antibody-coated microcantilevers are capable of sensing interactions with antigens (Grogan, 2002), and can even count the number of bacteria on the cantilever by monitoring the shift in the cantilever's resonant frequency (Ilic, 2000).

\section{Chemical Sensing}

Liquid and chemicals can be easily detected when cantilevers are coated with chemically selective thin-films layers. Gold coated, silicon nitride AFM cantilevers have been shown to deflect based on the $\mathrm{pH}$ and salinity of the surrounding liquid (Butt, 1995) and the sensitivity of $\mathrm{pH}$ detection has been improved by using chemically modified microcantilevers (Ji, 2001). It is also possible to selectively detect very minute concentrations of metal ions using cantilevers coated in selective self-assembled response layers (Cherian, 2002; Ji, 2000; Ji, 2001). Cantilevers have even been modified with 
synthetic receptor compounds to detect various neutral aromatic compunds in aqueous solution (Tipple, 2002).

Gaseous chemicals can also be detected using specially coated microcantilevers. Gold and palladium coated cantilevers are capable of detecting mercury vapor (Thundat, 1995) and hydrogen gas (Lang, 1999). Detection of mercury vapor by Thundat et al. (1995) was one of the first gas sensor applications of microcantilevers. Their gold-coated silicon nitride cantilevers deflected due to an increase in mass when the gold absorbed mercury vapor. The changes in resonant frequency were not reversible because the mercury and gold formed an amalgam. PMMA has been used as a microcantilever coating for the detection of different alcohols (Lang, 1998). Microcantilevers have also been coated in organic thin-films to detect humidity and other vapors (Thundat, 1995).

Lang et al. (1998) developed a "chemical nose" based on a microcantilever array containing eight cantilevers. Each cantilever was coated with a different material to detect specific analytes, such as alcohols and $\mathrm{H}_{2}$. However, the array used an optical lever detection method which added expense and bulk to the measurement setup. Also, the measurement signal was extremely noisy and required a comparison of the sensing cantilever to a reference cantilever in order to determine the signature of the analyte.

Battiston et al. (2001) also developed a chemical sensor based on a simple array of eight microcantilevers. These cantilevers were vertically actuated with a piezoelectric crystal and movement was detected with a vertical-cavity surface-emitting laser (VCSEL) paired with a position-sensitive detector (PSD). Different polymer materials were applied to the cantilevers in droplets and dried. Each coating was most sensitive to the solvent typically used to dissolve the polymer. They were able to detect water, primary alcohols 
(butanol, propanol, ethanol, and methanol), alkanes (hexane, heptanes, octane, nonane, decane, undecane, and dodecane), and certain perfume oils in both dynamic and static actuation modes. They were also able to release the analyte from the polymer absorption layer by purging the test chamber for some time with dry nitrogen. However, this group used the bulky and costly optical lever detection method paired with only one PSD. This meant that the eight VCSELs were time-multiplexed at a frequency of $3 \mathrm{~Hz}$ so that only one incident light source was switched on at a time, resulting in sequential displacement detection with the PSD. While this may not have delayed the signals by much time, the data points for each cantilever were sampled at different points in time, not simultaneously. A better approach for high-speed detection would be to collect data from each microcantilever simultaneously.

\section{Detection Methods}

Any cantilever sensor operates on the principle that it can detect accurate, real-time measurements of cantilever deflection. Detection is performed by monitoring one of the cantilever beam parameters for a change corresponding to deflection, such as the resonator tip position or radius of beam curvature.

\section{Optical Lever Method}

By far the most common method of determining the deflection of a cantilever is the optical lever technique (Meyer, 1988). As shown in Figure 1, a laser is focused on the end of the cantilever, which acts as a mirror to reflect the laser onto a position sensitive photodetector (PSD). A change in deflection of the cantilever will move the laser on the PSD, whose change in output is proportional to the deflection of the cantilever. 


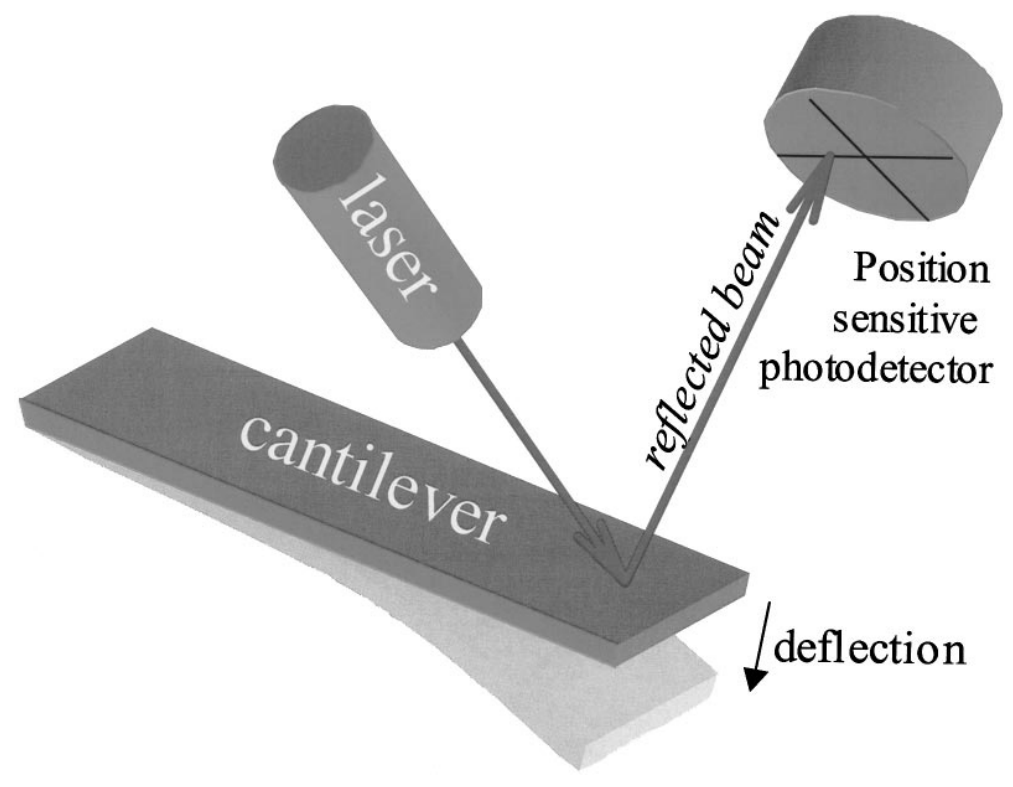

Figure 1 - Schematic of "Optical Lever" Detection Method (Lavrik, 2004).

This method has several advantages when compared to other techniques. It is characterized by a linear response and is very reliable. Also, cantilever beams that are non-conductive can be used with the optical lever technique. One major limitation is that this method cannot be used with portable systems because of the bulky optical components which must be finely aligned to the cantilever. Also, this method is limited by the bandwidth of the PSDs, which is around several hundred kilohertz (Lavrik, 2004).

\section{Piezoelectric Method}

Piezoelectric cantilever detection methods require that a piezoelectric substance be deposited on the microcantilever. Some common piezoelectric materials used in MEMS fabrication are lead zirconium titanate (PZT) (Gaucher, 1998; Furukawa, 1979) and crystalline zinc oxide $(\mathrm{ZnO})(\mathrm{Xu}, 2003)$. The principle behind this type of detection is that the cantilever deforms and the piezoelectric material generates an electric charge that can be measured by readout circuitry (Gaucher, 1998; Lee, 2003; Wang, 2003). 
Similar to piezoresistive detection, an advanatage to piezoelectric detection is that there are no external optics or external actuators needed for detection. The piezoelectric material serves as both the actuator and the sensor. The main disadvantage of this technique is that the piezoelectric layer must be thick enough to generate an adequately large output signal. This requirement usually requires a thickness above the value for adequate mechanical operation of the microcantilever. In addition, this method is not as effective when the cantilever operates at low frequencies.

\section{Capacitive Method}

The capacitive sensing method is based on the principle that the capacitance between two electrodes is inversely proportional to the distance between the plates. For capacitive cantilever detection, the cantilever is used as an electrode and a fixed conductor on the supporting substrate is used as the second electrode. When the cantilever deforms, the gap between the cantilever and electrode changes, changing the capacitance (Brugger, 1992; Abadal, 2001). For a large output signal, the gap between the cantilever and the fixed electrode is usually very small. The main advantage of the capacitance method is that it is very sensitive and measures the absolute displacement of the cantilever. A disadvantage of this method is that it can only be used to measure small displacements.

\section{Piezoresistive Method}

Piezoresistivity is a phenomenon in which the bulk resistance of a material changes with applied stress. This property can be exploited to measure the deformation of a resonator made of a piezoresistive material by monitoring the change in resistance. One 
common micromachining material that exhibits a strong piezoresistive effect is doped silicon (Brysek, 1991; Tufte, 1963).

One advantage of piezoresistive detection compared to optical detection is the elimination of expensive optical components and laser alignment steps. A second possible advantage is the integration of read-out electronics on the same chip as the cantilever using CMOS fabrication technology. Another advantage is that piezoresistive detection works in non-transparent solutions.

The primary disadvantage of piezoresistoive detection is the Joule heating effect. The current flowing though the resistor generates heat and thus causes additional dissipation of heat and thermal drift. This can be partially overcome by including another cantilever in the Wheatstone bridge which acts as a reference cantilever and is influenced by the same thermal environment as the sensing cantilever. By measuring the differential signal between the sensing cantilever and the reference cantilever, the thermal drift can be mostly eliminated from the measurements (Thaysen, 1999).

\section{Piezoresistive Microcantilever Sensors}

The piezoresistive cantilever technique was first reported by Tortonese (1991), and has been utilized by several different groups since then (Chui, 1998; Yuan, 1994; Willemin, 1998; Abedinov, 2001; Porter, 2001; Xu, 2006). Piezoresistive cantilevers are usually designed to include two identical piezoresistor "legs". By attaching the cantilever to the piezoresistive element, the bending of the cantilever causes stress in the piezoresistive element which can be monitored through the resistance change of the piezoresistor. The change in resistance is typically measured by including the cantilever in a DC-biased Wheatstone bridge (Tortonese, 1993; Gel, 2004). 


\section{E. Reaction Versus Non-Reaction Microcantilever Sensors}

Most microcantilevers used as sensors rely on an addition of mass to the cantilever for sensing purposes. The cantilever beam is coated with a material that functionalizes it or the cantilever beam itself is capable of absorbing an analyte (Battiston, 2001). The added mass is detected statically by monitoring beam deformation and dynamically by monitoring resonance frequency changes (Lavrik, 2004). Static cantilever deflections are either caused by external forces exerted on the beam, such as in an Atomic Force Microscope (AFM), or intrinsic stresses generated on the beam surface or within the beam. Intrinsic stresses may be caused by thermal expansion or physisorption/chemisorption processes. Dynamic cantilevers are essentially acting as mechanical oscillators. The resonance characteristics depend upon the beam mass and the viscoelastic properties of the surrounding medium. Absorption of analyte molecules on a resonating cantilever causes a reduction in resonant frequency due to an increase in beam mass.

The reactions used to absorb analytes are typically permanent (Kooser, et al. 2003) and limited by the availability of suitable gas reaction mechanisms, severely limiting the life and potential applications of the sensors. These microcantilever sensor reactions can sometimes be reversed by applying a vacuum, with a nitrogen gas purge, or by applying heat. The reaction-based microcantilever sensor's life is limited to the life of the functionalized surface.

The alternatives to reaction-based microcantilever sensors are cantilever sensors that perform detection without a physisorption or chemisorption process (Xu, Lin, et al. 2006). Non-reaction-based cantilever sensors are capable of measuring physical 
parameters in the surrounding environment that do not rely on chemical reactions, such as temperature (Thundat, 1995), acceleration (Morante, 1996), gas flow velocity (Su, 2002), and viscosity (Agoston, 2005). One of these non-chemical physical interactions that influence a resonating microcantilever is the damping force caused by gas particles in the surrounding environment.

\section{Viscous Damping of a Resonating Microcantilever}

Blom et al. (1992) calculated the theoretical shift in resonance frequency of a microcantilever due to the viscous damping effect from a surrounding gas and demonstrated that the shift is a function of the gas pressure and molar mass of the gas. The molar mass of the gas can be obtained by measuring the resonance frequency shift due to the damping effect, thus yielding the gas or the composition of the gases in the environment. Blom theoretically analyzed the effect of damping in a gaseous environment on the resonance frequency and quality factor of microresonators. At higher pressure, in the viscous regime, the inertial force of the gas on the resonator creates a damping effect, which lowers the resonance frequency. The relative resonance frequency shift in the viscous regime is given by

$$
\frac{\Delta f}{f}=\frac{\pi R^{3}}{3 m_{b} R_{0} T}\left(M P+\frac{9}{2} \frac{\sqrt{\mu R_{0} T / \pi f}}{R} \sqrt{M P}\right)
$$

where $\mathrm{m}_{\mathrm{b}}$ is the mass of the cantilever, $\mathrm{R}_{0}=8.314 \mathrm{~J} /(\mathrm{mol} \bullet \mathrm{K})$ is the gas constant, $\mathrm{T}$ is the absolute temperature, $\mathrm{M}$ is the molar mass of the gas, $\mathrm{P}$ is the pressure, $\mu$ is the dynamic viscosity of the gas, $\mathrm{f}$ is the resonance frequency in vacuum, and $\Delta \mathrm{f}$ is the shift of the resonance frequency. The cantilever is approximated by a string of spheres, with $\mathrm{R}$ equal to the radius of one of the spheres. The inertial force of the gas is proportional to the product of the mass times the acceleration of the gas in contact with the cantilever. 
The relative resonance frequency shift due to inertial damping is thus dependent on the molar mass of the gas in the environment. By measuring the relative resonance frequency shift at a specific temperature and pressure (e.g., room temperature and atmospheric pressure), the molar mass of the unknown gas can be obtained.

\section{F. Previous Work With Symmetric Microcantilevers}

Xu et al. (2006) demonstrated a remarkable piezoresistive cantilever beam capable of gas detection solely through viscous damping of a laterally vibrating beam. The piezoresistive sensing opens up the possibilities for on-board signal processing by integrating the circuitry onto the same silicon chip with CMOS technology. The magnitude of the viscous damping was found to be directly determined by the molar mass of the surrounding gas. This technique avoids the difficult alternative of coating the microcantilever with a gas-sensitive polymer, and also allows the detection process to be simple, fully reversible, and capable of detecting non-reactive gases.

However, Yang's microcantilever beams had relatively low sensitivity to the surrounding gases; the resonant frequency of the microcantilever $(\sim 42,000 \mathrm{~Hz})$ changed by only $20 \mathrm{~Hz}$ when the $\mathrm{CO}_{2}$ concentration was changed by $20 \%$, resulting in a $0.05 \%$ change in the resonant frequency. Yang's best device sensitivity was $0.0231 \Omega / \mu \mathrm{m}$ and her T-9 resonators had an average relative resistance change of $2.7 \times 10^{-6}$ for a $1 \mu \mathrm{m}$ deflection of the free end of the cantilever. This $10^{-6}$ relative resistance change approaches the detection limit of the Wheatstone bridge configuration used to measure the change in piezoresistance. This also resulted in a low signal-to-noise ratio, especially at atmospheric pressure with ambient lighting. One alternative to increase the device sensitivity is to decrease the length of the cantilever, since, according to Equation 1 the 
relative frequency shift is inversely proportional to the mass and hence to the length of the cantilever. Yang's work can be further improved and built-upon by incorporating similarly designed microcantilevers into an array for redundancy and selectivity, and by optimizing the cantilever geometry to improve device sensitivity.

Current piezoresistive and piezoelectric microcantilevers rely on symmetric geometry to measure changes in cantilever mass or surface stress. These measurements are carried out by measuring the stress in the piezo element. Bending stresses in the support structure of the cantilever, which occur when the cantilever tip is displaced, cause changes in piezoresistance. However, the beneficial axial stresses largely cancel due to the nature of the symmetric geometry of the cantilever base. An alternative method for increasing the stress in the piezo element must be developed. The geometry of the cantilever base can be optimized, thereby improving the resistance sensitivity. An unutilized alternative is to vary the leg stagger and leg length of the piezoresistive element, creating an asymmetric piezoresistive element with favorable coupling stresses. Optimizing the stagger of the cantilever base legs and the length of the base legs should maximize the bending and axial loads on the base legs. Both types of loads produce a change in resistance and the optimized, asymmetric geometry will improve the overall device sensitivity.

Compared to other microresonator gas sensors, there are several major advantages of the asymmetric, lateral microcantilever sensor developed in this study, including:

- A simple fabrication process that does not require a specific material for the resonator or any chemically sensitive coatings.

- Greater sensitivity than previous T-shaped designs. 
- Non-reactive and inert gases can be detected.

- The detecting process is reversible and repeatable.

- Detection can be performed at atmospheric pressure.

- The device and measurement circuitry can be integrated on a chip using MEMS and CMOS technology.

- An array of resonators can be used to detect particular analytes and can serve as redundant systems.

Computational studies (Bradshaw, et al. 2007) have indicated that Yang's symmetric device sensitivity can be increased up to 700 times, purely by optimizing the geometry of the base piezoresistive support structure. The purpose of this work is to report on the fabrication and experimental testing of these novel asymmetric piezoresistive microcantilevers. 


\section{III.MATERIALS AND METHODS}

Current piezoresistive and piezoelectric microcantilevers rely on symmetric geometry to measure changes in cantilever mass or surface stress. These measurements are carried out by measuring the stress in the piezo element. A laterally resonating piezoresistive cantilever with T-shaped geometry (Figure 2) has been fabricated and tested in our group, but the resonating device sensitivity was low (Xu, Lin, et al. 2006).

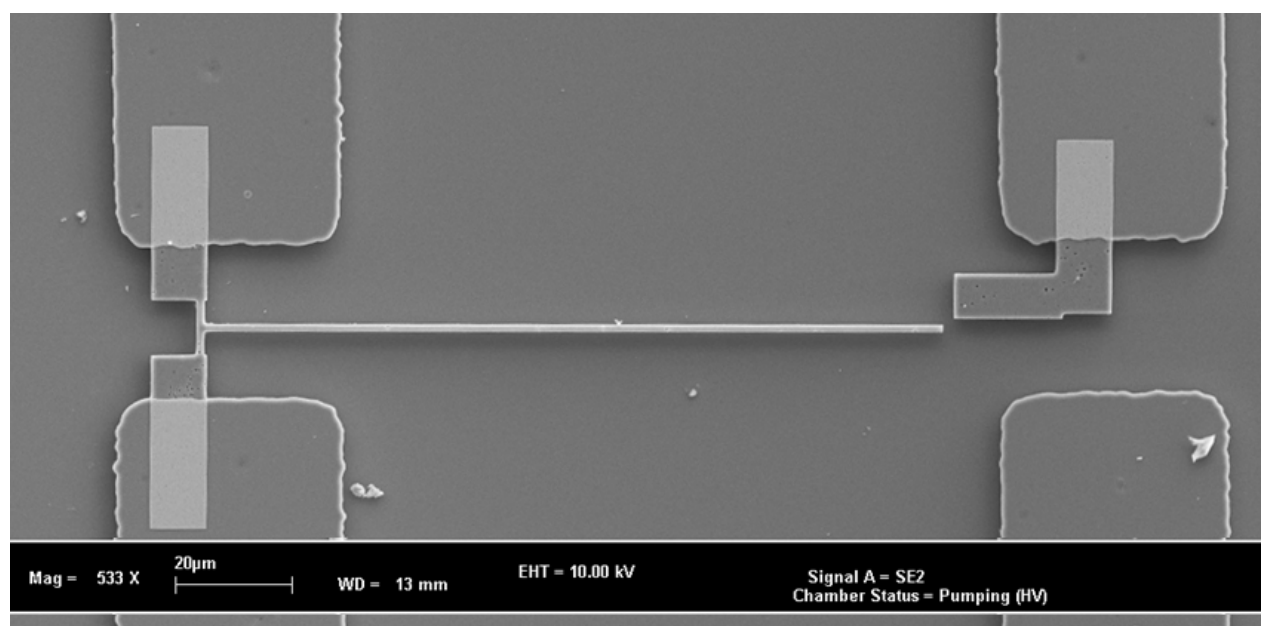

Figure 2 - Laterally Vibrating Silicon Microcantilever with Symmetric, T-Shaped Piezoresistive Element (Xu, 2006).

\section{A. Design}

\section{Computational Studies}

Bradshaw et al. (2007) showed that the changes in resistance for the symmetric, laterally resonating microcantilever were due to bending stresses in the support structure of the cantilever, which occurred when the cantilever tip was displaced. However, the beneficial axial stresses largely canceled due to the nature of the symmetric geometry of the cantilever base. The axial loads are equal and opposite in each piezoresistor leg and the moments are equal and opposite through the cross section, causing the beneficial 
stresses to largely cancel each other out. A free-body diagram of the symmetric piezoresistive element is shown in Figure 3.

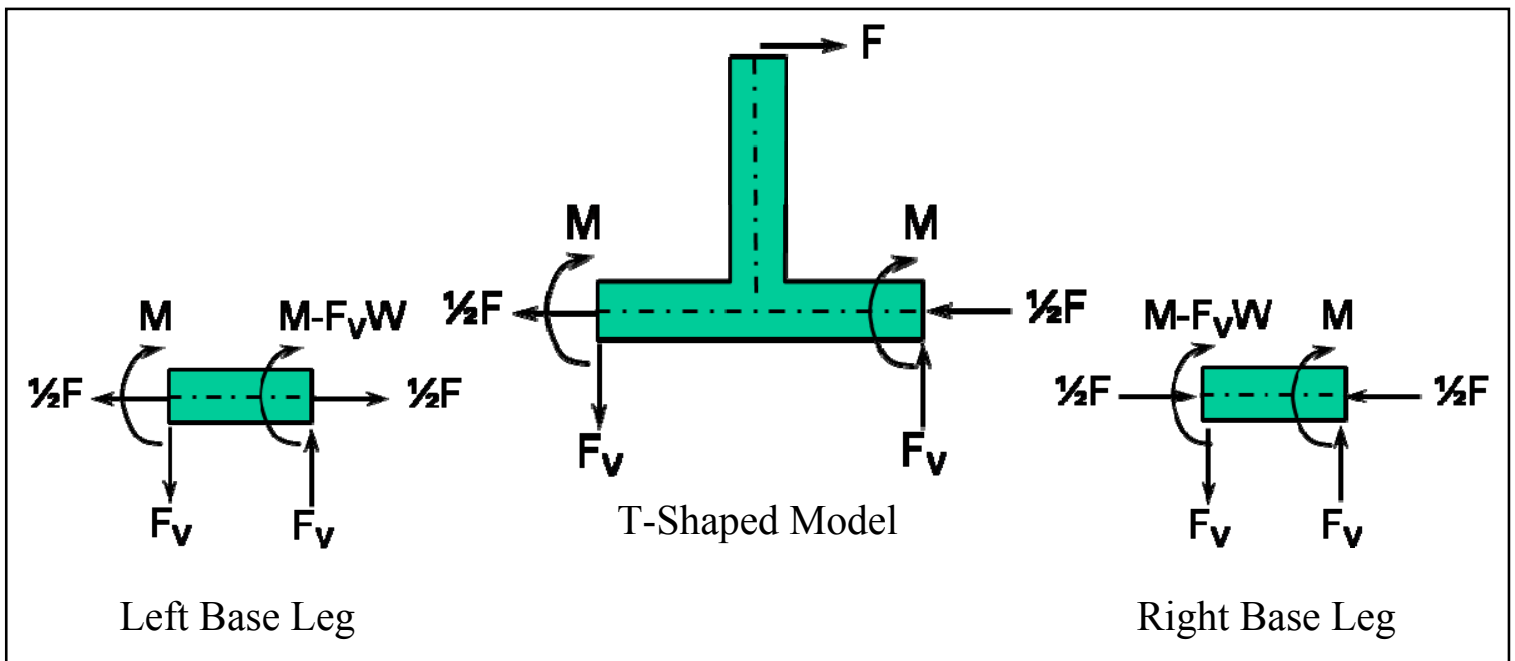

Figure 3 - Free-Body Diagram of T-Shaped Piezoresistive Element (Bradshaw, et al. 2007).

To increase the sensitivity of the cantilever, the relative change in stress in the piezo element must be increased when the cantilever is deflected. Currently, the stress in the piezo element can be increased by lengthening the cantilever, increasing stress concentration with sharper corners, and increasing the deflection of the resonating cantilever. However, there are several limitations to these methods:

- Increasing the length of the cantilever adds mass to the beam, which decreases the natural frequency. This leads to a lower Q-factor.

- It is hard to consistently produce sharp features with current micromachining techniques with a minimum feature size of $1 \mu \mathrm{m}$.

- Increasing the deflection of the cantilever requires a larger $\mathrm{AC}$ drive voltage, which means more power consumption and more equipment.

An alternative method for increasing the stress in the piezo element must be developed. The geometry of the cantilever base can be optimized, thereby improving the 
resistance sensitivity. Bradshaw et al. (2007) pointed out that an unused alternative is to vary the leg stagger and leg length of the piezoresistive element, creating an asymmetric piezoresistive element with favorable coupling stresses. The axial stresses that largely canceled in the symmetric model will form a force couple induced by the leg separation, placing both piezoresistive legs in tension or compression at the same time and leading to much larger changes in piezoresistance. A free-body diagram of an asymmetric piezoresistive element is shown in Figure 4.

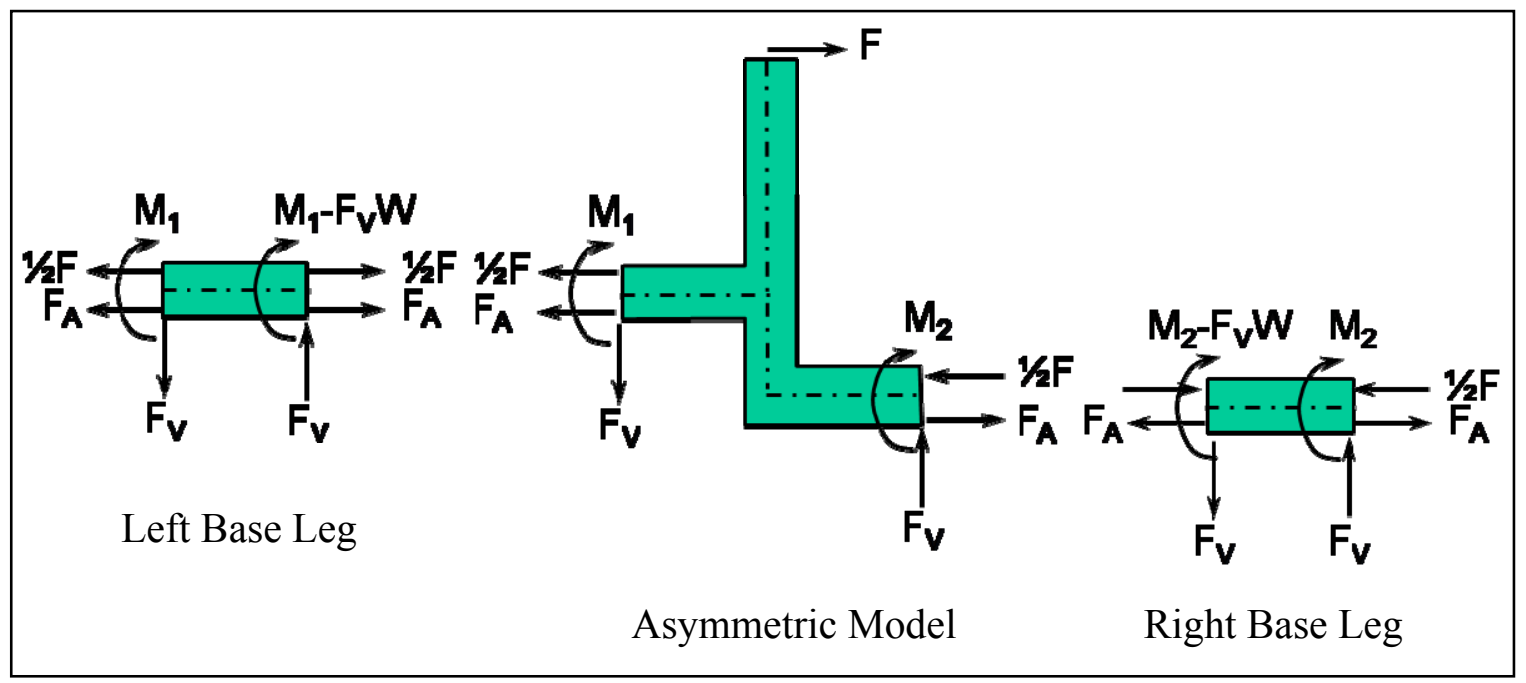

Figure 4 - Free-Body Diagram of Asymmetric Piezoresistive Element (Bradshaw, et al. 2007).

A finite element analysis (FEA) model was developed to determine the optimal piezoresistor geometry for fabrication. The model was built in $2 \mathrm{D}$ using an assumption of plane stress, and in 3D. PLANE223 and SOLID226 coupled field solid elements were used for 2D and 3D models, respectively, in the FEA computer package (ANSYS, Canonsburg, PA) to develop a parametric model with varying beam length, piezoresistor leg lengths, piezoresistor leg offsets, fillet radii, and silicon crystal orientation. The symmetric microcantilever geometry terms are explained in Figure 5. The piezoresistor ends were fixed in all degrees of freedom and a voltage drop of 5 Volts was applied 
across the piezoresistor. The tip of the cantilever beam was displaced $10 \mu \mathrm{m}$ when loaded. The resulting current change during loading was used to find the resistance change of the piezoresistor during actuation.

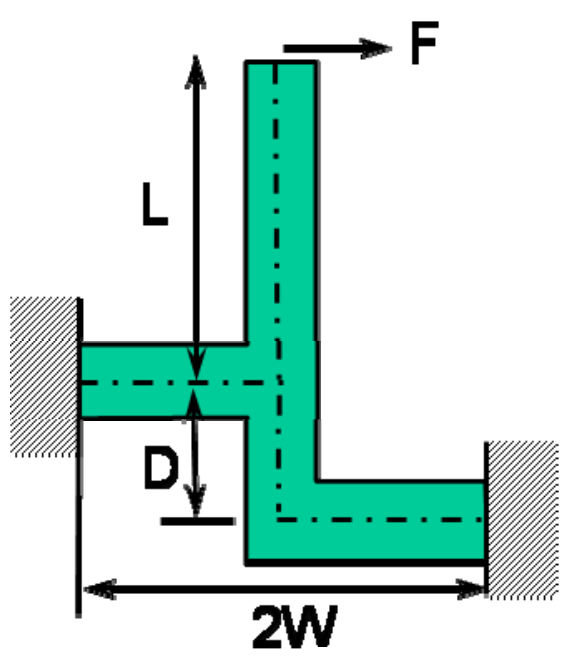

Figure 5 - Symmetric Microcantilever Geometry.

The model was run iteratively in a loop to test many piezoresistor designs and thus find the optimal geometry for the piezoresistor. The normalized resistance change for the symmetric piezoresistor with varying leg widths is shown in Figure 6. The normalized resistance change for the asymmetric piezoresistor with varying leg separations and piezoresistor widths is shown in Figure 7. 


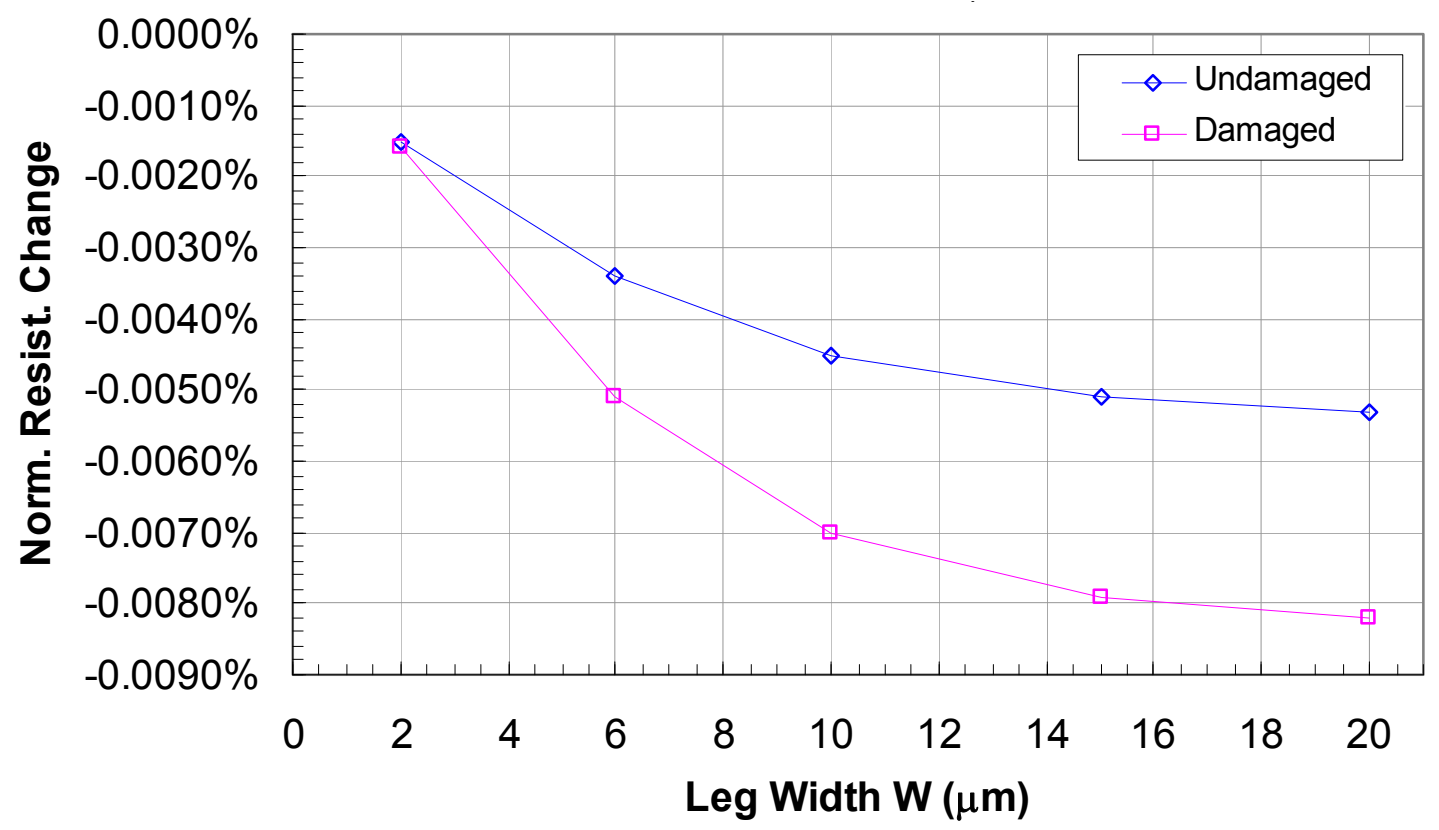

Figure 6 - Normalized Resistance For T-Shaped Model in $<110>$ Orientation and $1.1 \mu \mathrm{m}$ Thick Piezoresistor (Bradshaw, et al. 2007).

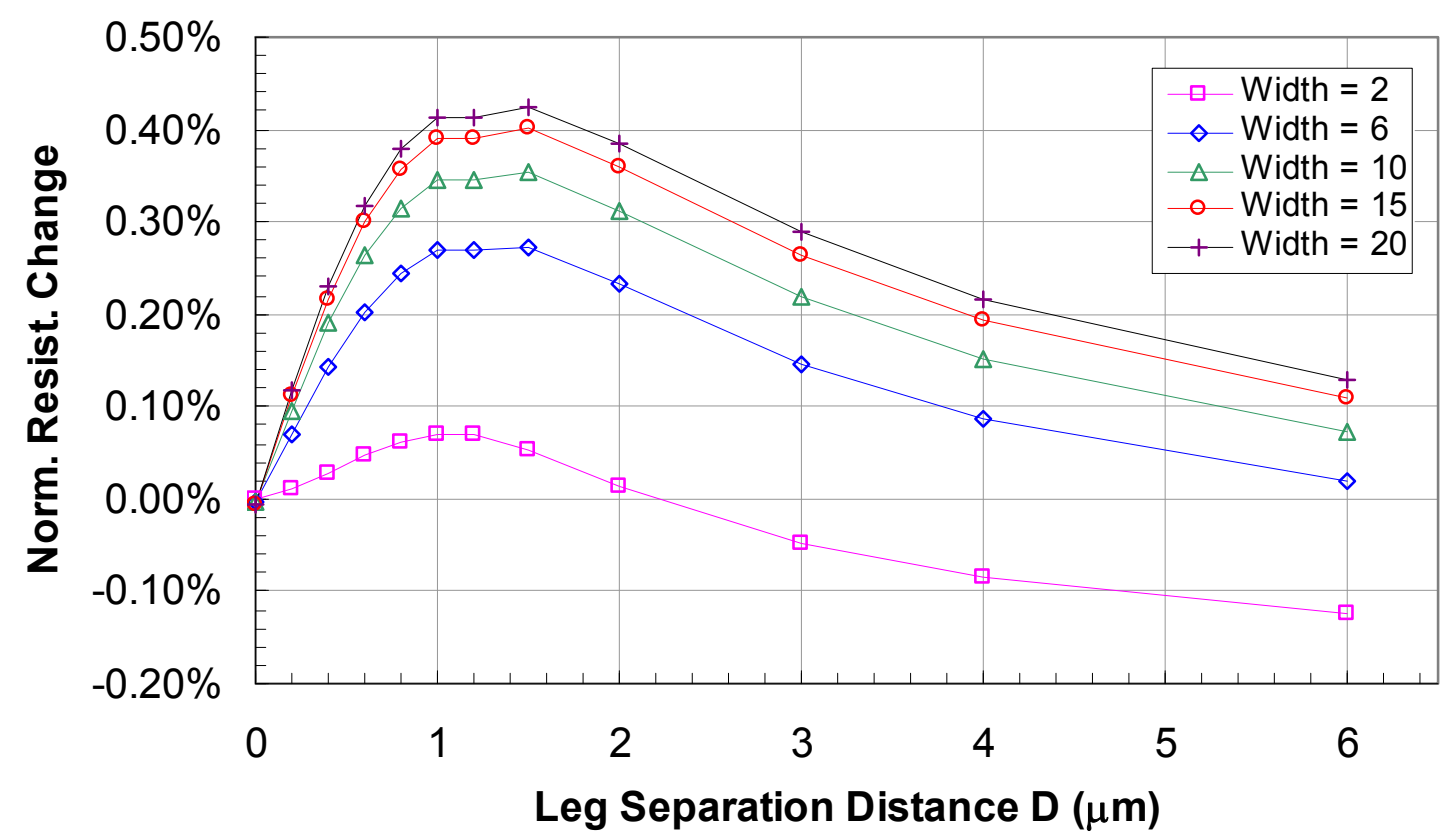

Figure 7 - Normalized Resistance for Asymmetric Model in $<110>$ Orientation and 1.1 $\mu \mathrm{m}$ Thick Piezoresistor (Bradshaw, et al. 2007).

The optimum geometry was analytically determined (Bradshaw, et al. 2007) to have a leg separation of $1 \mu \mathrm{m}$ and leg lengths of $20 \mu \mathrm{m}$. The asymmetric microcantilever geometry is shown in Figure 8. Optimizing the stagger of the cantilever base legs and the 
length of the base legs should maximize the bending and axial loads on the base legs. Both types of loads produce a change in resistance and the optimized, asymmetric geometry should improve the overall device sensitivity.

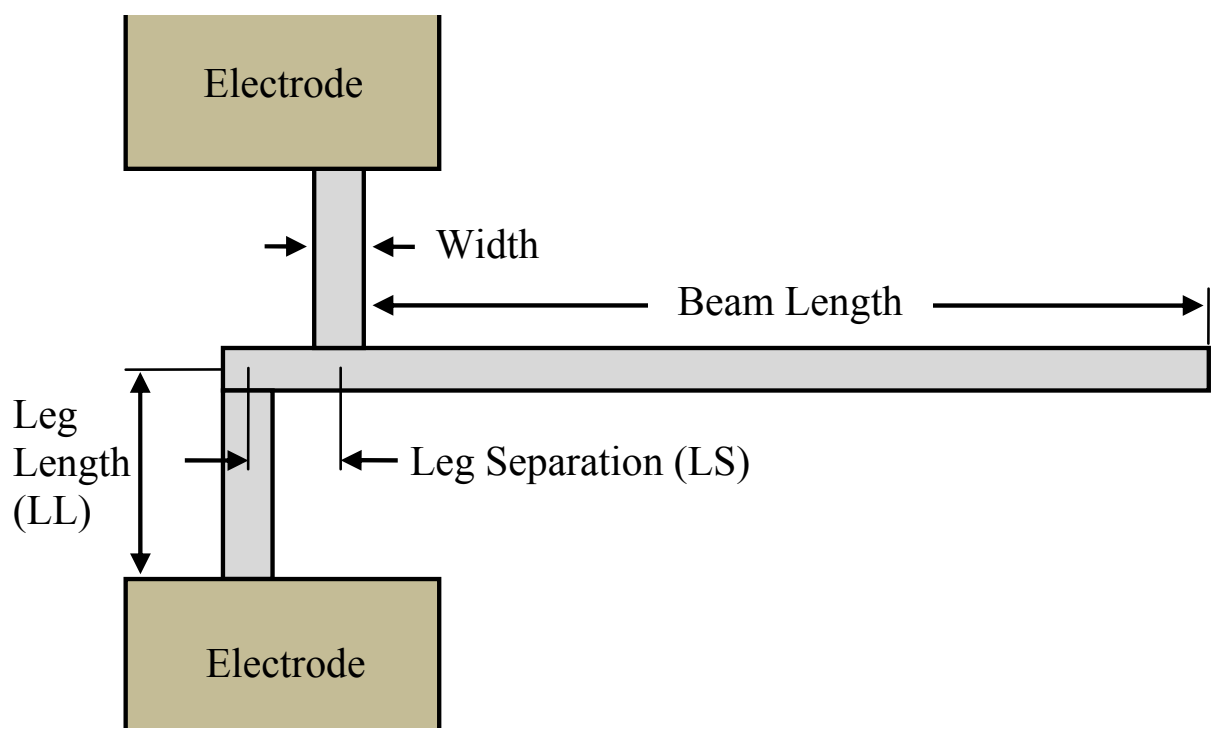

Figure 8 - Asymmetric Microcantilever Geometry.

Ten different piezoresistive microcantilever designs were formulated to verify the improved sensitivity of the optimum designs. Several variations of the asymmetric cantilever geometry were fabricated by varying the leg separation (LS), the leg length (LL), and the total piezoresistor length (PL). The PL is defined as

$$
\mathrm{PL}=\mathrm{LS}+2 * \mathrm{LL} .
$$

The microcantilever dimensions are listed in Table I. Beam 1 used Xu's (2006) exact Tshaped geometry to serve as a control to compare against earlier, less sensitive designs. Beam 2 used the optimum piezoresistor geometry (see Table I for all dimensional values). Beams 3 and 4 used optimum piezoresistor geometry with two variations to maintain the optimum PL, but vary LL and LS. Beam 5 was a microcantilever with a wider piezoresistive element (thickness $=1.3 \mu \mathrm{m}$ ) to determine whether Deep Reactive 
Ion Etching (DRIE) causes electrical damage in the piezoresistor. Beams 6 and 7 had optimal piezoresistor geometry with shorter $(110 \mu \mathrm{m})$ and longer $(150 \mu \mathrm{m})$ cantilever beams. Beam 8 was a piezoresistor design with the optimum LS, but shorter PL. Beams 9 and 10 were piezoresistor designs with the shorter PL, but varied LL and LS.

\section{TABLE I}

ASYMMETRIC MICROCANTILEVER GEOMETRY DESIGNS

\begin{tabular}{|c|c|c|c|c|c|}
\hline Beam Number & $\operatorname{LS}(\mu \mathrm{m})^{\mathrm{a}}$ & $\operatorname{LL}(\mu \mathrm{m})^{\mathrm{a}}$ & PL $(\mu \mathrm{m})^{\mathrm{a}}$ & $\mathrm{PW}(\mu \mathrm{m})^{\mathrm{a}}$ & CL $(\mu \mathrm{m})^{\mathrm{a}}$ \\
\hline 1 & - & 4.5 & 9 & 1.1 & 128 \\
\hline $2^{\mathrm{b}}$ & 1 & 20 & 41 & 1.1 & 128 \\
\hline 3 & 2 & 19.5 & 41 & 1.1 & 128 \\
\hline 4 & 4 & 18.5 & 41 & 1.1 & 128 \\
\hline 5 & 1 & 20 & 41 & 1.3 & 128 \\
\hline 6 & 1 & 20 & 41 & 1.1 & 110 \\
\hline 7 & 1 & 20 & 41 & 1.1 & 150 \\
\hline 8 & 1 & 10 & 21 & 1.1 & 128 \\
\hline 9 & 2 & 9.5 & 21 & 1.1 & 128 \\
\hline 10 & 4 & 8.5 & 21 & 1.1 & 128 \\
\hline
\end{tabular}

a. $\mathrm{LS}=$ leg separation, $\mathrm{LL}=$ leg length, $\mathrm{PL}=$ piezoresistor length, $\mathrm{PW}=$ piezoresistor width, $\mathrm{CL}=$ cantilever length

b. The optimum geometry

The device layout (Figure 9) has 10 microcantilever beams arranged in an array on a silicon wafer. Each microcantilever has a driving electrode and the piezoresistive element is a freely-suspended, boron-doped silicon bridge positioned between two sensing electrodes. 


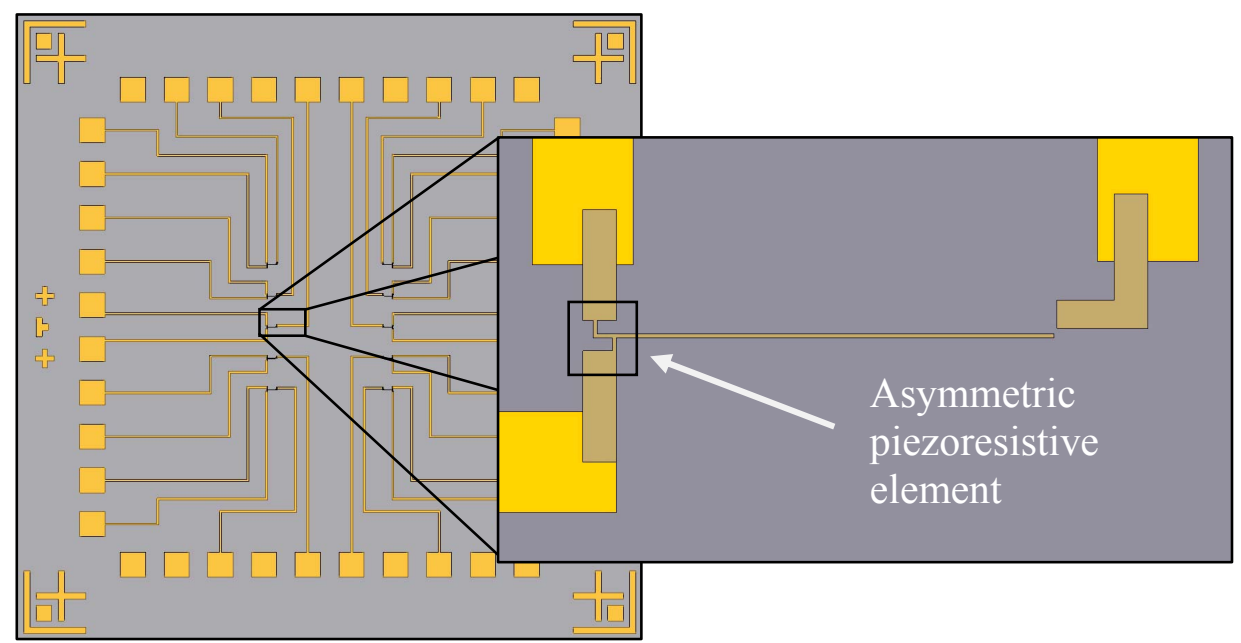

Figure 9 - Device Layout with 10 Microcantilever Beams in an Array.

B. Fabrication

The fabrication process for this device began with a commercial, 4" silicon-oninsulator (SOI) wafer (Ultrasil Corporation, Hayward, CA). This wafer was comprised of three layers. The top layer was called the device layer and was comprised of a $2 \mu \mathrm{m} \pm 0.5$ $\mu \mathrm{m}$ thick layer of boron-doped (resistivity $0.01-0.02 \Omega \cdot \mathrm{cm}$ ) crystal silicon with a $<100>$ orientation. The middle layer was composed of buried silicon dioxide, acting as an electrical insulator, and was $2 \mu \mathrm{m} \pm 0.1 \mu \mathrm{m}$ thick. The bottom layer, or handle layer, was $510 \mu \mathrm{m} \pm 10 \mu \mathrm{m}$ thick, which also consisted of a boron-doped (resistivity $0.01-0.02 \Omega$ $\cdot \mathrm{cm})$ crystal silicon wafer. A base clean was performed to remove most impurities from the surface of the wafer and to strip away the native oxide on the silicon. This cleaning consisted of a 5 minute rinse in acetone, followed by a 5 minute soak in nanostrip and a rinse in de-ionized (DI) water. Finally, the wafer was soaked in a buffered oxide etch (BOE) for 30 seconds to remove any remaining native oxide and rinsed in DI water.

The overall fabrication process is illustrated in Figure 10. As an overview, the gold bond pads and leads were patterned using optical lithography, gold sputtering, and acetone lift-off techniques (Figure 10b). The iron masking layer for the dry etching 
process was then patterned using e-beam lithography, evaporation, and acetone lift-off techniques (Figure 10c). Next, the silicon device layer was dry etched (DRIE) using the gold and iron as masks (Figure 10d). In conclusion, the sacrificial silicon dioxide layer was wet etched, releasing the structure, and the device was cleaned and dried with critical point drying (Figure 10e). Each fabrication step is discussed below in further detail.

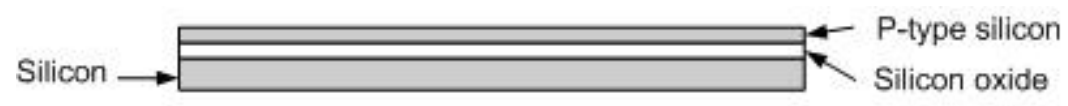

(a)

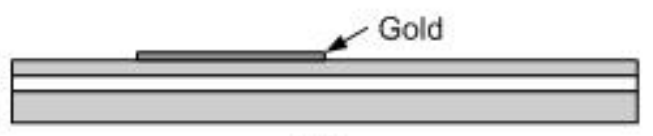

(b)

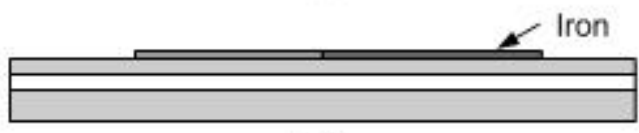

(c)

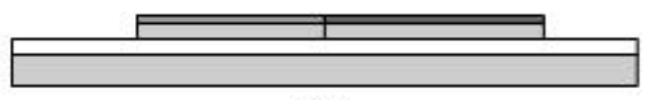

(d)

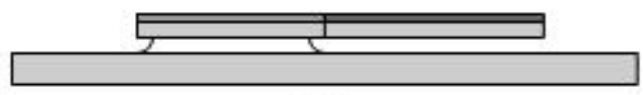

(e)

Figure 10 - Schematic for Fabrication Process (Xu, 2006).

\section{Optical Lithography}

Gold wire-bonding pads and electrical leads were fabricated using an optical lithography technique. Two lithography steps were required because the desired gold bond pads were thicker than the gold layer desired for the leads. Figure 11 shows the two masks used in the optical lithography process. 


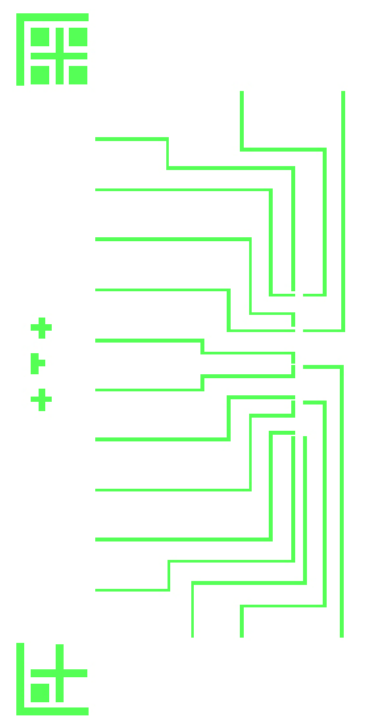

(a) Mask 1

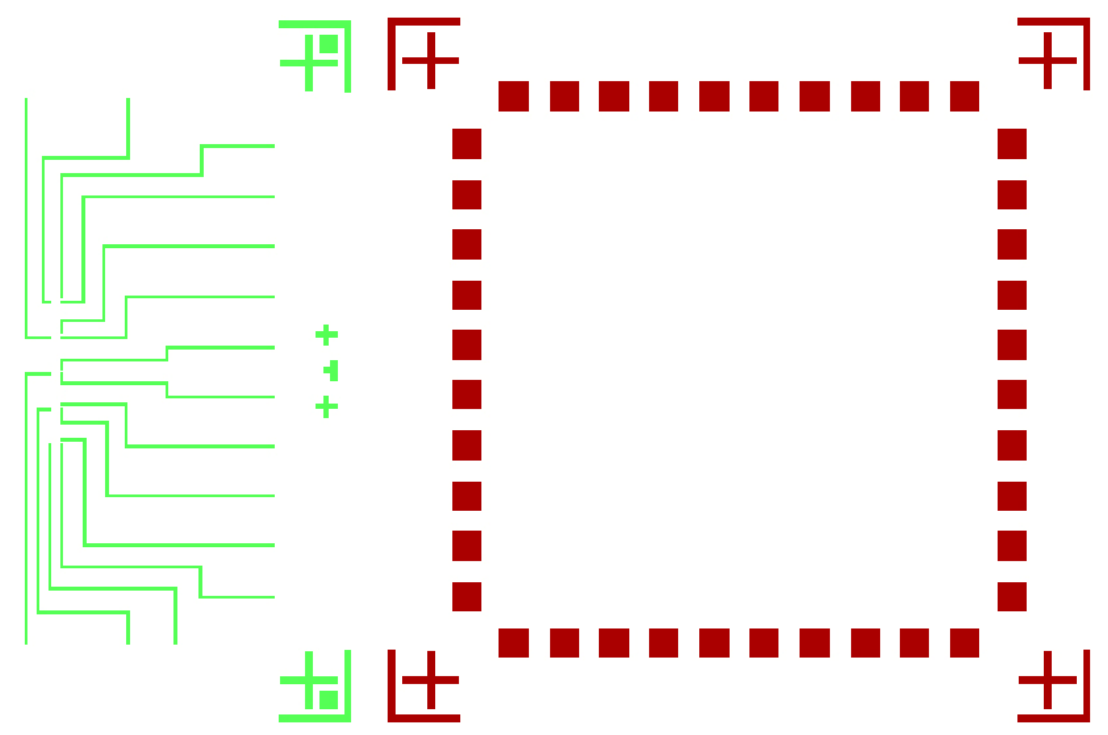

(b) Mask 2

Figure 11 - Design Layout of the Optical Lithography Mask Files. Mask 1 was used first to pattern the electrical leads, followed by mask 2 to pattern the bond pads.

To begin the photolithography process, the SOI wafer was 'baked' for 5 minutes at $115^{\circ} \mathrm{C}$ on a hotplate to remove excess moisture and promote photoresist adhesion. LiftOff Resist 3A (LOR3A, Microchem Corp., Newton, MA) was then applied to the silicon surface using a wafer spinner at a spread speed of $450 \mathrm{rpm}$ for 2.0 seconds and a spin speed of $3000 \mathrm{rpm}$ for 10 seconds to achieve a LOR3A thickness of $330 \mathrm{~nm}$.

Subsequently, the SOI wafer was baked on a hotplate for 5 minutes at $170^{\circ} \mathrm{C}$ with a vacuum contact to remove excess solvent from the resist. Shipley 1827 positive photoresist (Rohm and Haas Electronic Materials, LLC, Marlborough, MA) was then applied on top of the LOR3A using a spinner (the spread and spin speeds were the same as those used for the LOR3A) and soft baked at $115^{\circ} \mathrm{C}$ for 75 seconds to remove excess solvent from the positive resist.

Next, the substrate was exposed to UV light for 11 seconds using Mask 1 in a mask aligner (AB-M, Technical Manufacturing Corp., Peabody, MA). The resulting pattern 
was developed in MF 319 (Rohm and Haas Electronic Materials LLC, Marlborough, MA) for 90 seconds with varied lateral agitation and rinsed in DI water. Following drying, an adhesion layer of $10 \mathrm{~nm}$ thick chromium was RF sputtered (4604, Technics, Inc, Dublin, CA) onto the substrate at $350 \mathrm{~W}$ for 27 seconds $(\sim 0.3 \mathrm{~nm} / \mathrm{s})$ and a $35 \mathrm{~nm}$ thick layer of gold was DC sputtered onto the substrate at $120 \mathrm{~W}$ for 24 seconds $(\sim 1.5$ $\mathrm{nm} / \mathrm{s}$ ). After sputtering, a gold/chrome lift-off process was performed by submerging the wafer in a recirculating acetone bath for one hour to remove the excess gold and chromium, leaving the desired electrode lead pattern on the substrate. The wafer was rinsed in a DI water bath to remove excess acetone.

Optical lithography was performed a second time to create the bond pads. Again, the wafer was dehydration baked, LOR3A was applied and soft baked onto the substrate, Shipley 1827 was applied and soft baked onto the substrate using the same parameters mentioned previously in the first photolithography process. The substrate was exposed to UV light for 11 seconds on the AB-M mask aligner using Mask 2. The resulting pattern was developed in MF 319 for 90 seconds and rinsed in DI water. An adhesion layer of 10 $\mathrm{nm}$, thick chromium was RF sputtered onto the substrate at $350 \mathrm{~W}$ for 27 seconds and a $150 \mathrm{~nm}$ thick layer of gold was DC sputtered onto the substrate at $120 \mathrm{~W}$ for 103 seconds $(\sim 1.5 \mathrm{~nm} / \mathrm{s})$. After sputtering, a lift-off process was again performed in a recirculating acetone bath for one hour and the wafer was rinsed in DI water. The lift-off revealed the intricate gold leads and bond pads patterned on the substrate surface.

\section{Electron Beam Lithography}

The microcantilever array was fabricated using electron beam (e-beam) lithography because conventional contact optical lithography is not capable of producing the small, 
high resolution feature sizes needed. Prior to e-beam lithography, the 4" SOI wafer was diced on a dicing saw (DAD 321, Disco Hi-Tec America, Inc., Manchester, NH) so that each individual SOI substrate contained one die. To begin the e-beam lithography process, the diced SOI substrate was cleaned with acetone in an ultrasonic bath for 10 seconds, rinsed in isopropyl alcohol (IPA), and blown dry with nitrogen. The substrate was then dehydration baked on a hotplate for 5 minutes at $115^{\circ} \mathrm{C}$. A copolymer positive resist (MMA 8.5 MAA EL9, Microchem Corp., Newton, MA) was applied to the substrate surface using a wafer spinner with a spread speed of $450 \mathrm{rpm}$ for 2.0 seconds and spin speed of $6000 \mathrm{rpm}$ for 40 seconds. Subsequently, the EL9 was baked to remove excess solvent at $180^{\circ} \mathrm{C}$ for 5 minutes. A positive resist (495 PMMA A5.5, Microchem Corp., Newton, MA) was spread on the substrate surface at $450 \mathrm{rpm}$ for 2 seconds and spun at $6000 \mathrm{rpm}$ for 40 seconds to produce. The $\mathrm{A} 5.5$ was baked on a hotplate at $180^{\circ} \mathrm{C}$ for 5 minutes. Finally, a third positive resist (950 PMMA A8, Microchem Corp., Newton, MA) was spread at $450 \mathrm{rpm}$ for 2 seconds and spun at $6000 \mathrm{rpm}$ for 40 seconds. The layer of $\mathrm{A} 8$ was baked on a hotplate at $180^{\circ} \mathrm{C}$ for 15 minutes.

The pattern for the e-beam lithography process was designed using Design CAD (IMSI, Novato, CA). Each cantilever was designed and saved in a separate file; as a result, each cantilever required alignment before e-beam writing. An example of the design file for a typical cantilever is shown in Figure 12. The exposure parameters of the anchors and the beam were different in order to speed up the writing process, to shorten the write time from 87 minutes to $\sim 9$ minutes. 


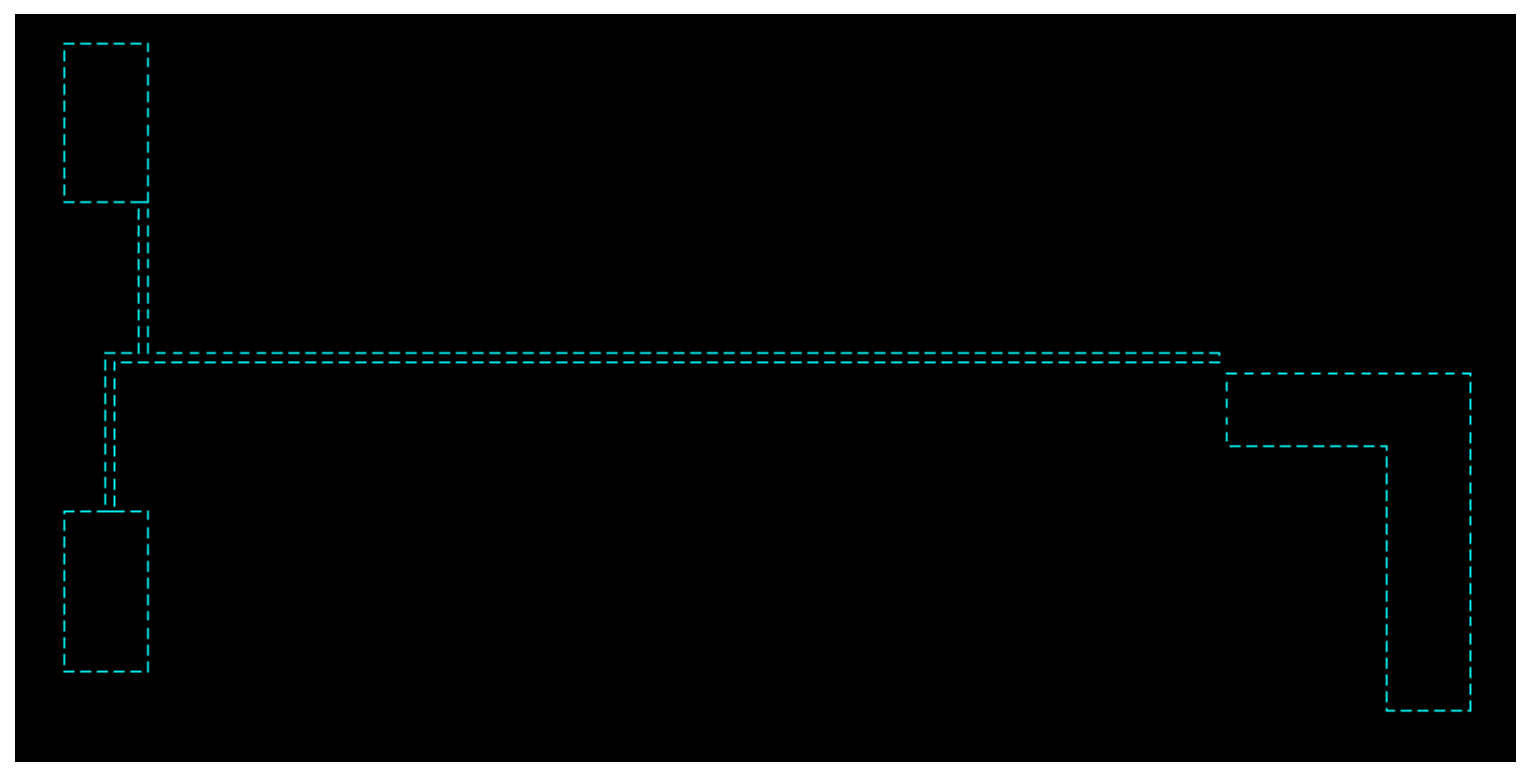

Figure 12 - Example of Cantilever Pattern for E-Beam Writing.

The three-layer photoresist-coated substrate was placed in a scanning electron microscope (SEM) (Model Zeiss LEO 1430, Carl Zeiss SMT AG, Germany) with a Nanometer Pattern Generation System (NPGS) to perform the e-beam writing procedure. The exposure parameters, such as magnification, dose, probe current, center-to-center distance and line width, were input in the Run-File of the NPGS. The anchor areas were exposed to a $20 \mathrm{kV}$ electron beam at a magnification of $125 \mathrm{x}$, a dose of $1.3 \mathrm{nC} / \mathrm{cm}$, a probe current of $80 \mu \mathrm{A}$, a center-to-center distance $1000 \AA$, and a line width of $2000 \AA$. In contrast, the beam areas were exposed at a dose of $1.3 \mathrm{nC} / \mathrm{cm}$, a probe current of $80 \mu \mathrm{A}$, a center-to-center distance $1400 \AA$, and a line width of $1400 \AA$.

The individual, exposed SOI die substrates were developed in methyl isobutyl ketone (MIBK) (Microchem Corp., Newton, MA) for 60 seconds and rinsed in isopropyl alcohol (IPA) for 30 seconds. An optical microscope was used to determine if the beam patterns were properly developed and whether additional development in MIBK was required. This development step created openings in the photoresist of the individual SOI die substrates down to the silicon device layer in the shape of the cantilever beams. A 5 
$\mathrm{nm}$ thick layer of iron was deposited on the sample surface using an electron-beam evaporator (Kurt J. Lesker Company, Pittsburgh, PA) at a pressure of $5 \times 10^{-7}$ Torr. Next, another lift-off process was performed in acetone, leaving the iron layer to act as a masking layer for the cantilever beams during the dry etching process.

\section{Dry Etching Process}

An anisotropic Deep-Reactive Ion Etch (DRIE, Multiple ASE Advanced Silicon Etcher, Surface Technology System USA, Inc., Newport, UK) process was performed to etch the silicon device layer of the SOI wafer because it is capable of forming high aspect ratio vertical sidewalls in silicon without etching the silicon-dioxide layer. The plasma was inductively coupled at $13.56 \mathrm{MHz}$ via a matching unit and coil assembly. Independent energy control was provided by a $13.56 \mathrm{MHz}$ biasing of the platen via automatic power control and a separate $380 \mathrm{kHz}$ generator. The platen was cooled by a DI water chiller and the backside of the substrate was cooled with helium gas.

The dry etch was performed with a base pressure of $0.2 \mathrm{mT}$ Torr and a process pressure of $10 \mathrm{mT}$ Torr at room temperature. An RF power of $600 \mathrm{~W}$ was applied by the 13.56 $\mathrm{MHz}$ generator for the etcher and the platen was powered by $15 \mathrm{~W}$ of RF power from the platen generator. The flow rate of the octafluorocyclobutane $\left(\mathrm{C}_{4} \mathrm{H}_{8}\right)$ was 75 sccm and the flow rate of the sulphurhexaflouride $\left(\mathrm{SF}_{6}\right)$ was $40 \mathrm{sccm}$. An in situ etching process with simultaneous passivation was chosen to generate the desired smooth sidewall features.

Prior to etching, each individual SOI die substrate was placed on a "dummy" silicon wafer that was coated with Shipley 1813 photoresist to prevent etching of the "dummy" wafer and it increased the etching surface area which reduced the overall etch rate of the 
process. The SOI die substrate was affixed to the "dummy" wafer with CoolGrease to improve heat transfer between the SOI die substrate and the "dummy" wafer as well as decrease the DRIE etch rate. The sulphurhexaflouride etched the exposed silicon surface while the reactant gas, octafluorocyclobutane, produced a protective polymer layer on the etched sidewalls to prevent further etching. The dry etching process was finished when the sacrificial silicon-dioxide layer was reached, indicated by a change in appearance from colorful $\mathrm{Si}$ to purple $\mathrm{SiO}_{2}$. The average etch rate was $\sim 0.17 \mu \mathrm{m} / \mathrm{min}$, which generally resulted in a processing time of 12 minutes for the $2 \mu \mathrm{m}$ thick silicon device layer. The etching process was performed in short time steps to prevent over etching to prevent damaging the cantilever beams. Specifically, the SOI die substrate was initially etched for 6 minutes and checked to see if the purple silicon dioxide was visible. If the silicon dioxide was not visible, the etching process was performed again for an additional 2 minutes. This process was repeated until the silicon dioxide was completely visible. After etching, the SOI die substrates were imaged and measured in an SEM (Model Zeiss LEO 1430, Carl Zeiss SMT AG, Germany).

\section{Wet Etching Process}

The cantilever beams were released from the substrate through an isotropic wet etching of the sacrificial $\mathrm{SiO}_{2}$ layer in a $\mathrm{BOE}$ to partially remove the $\mathrm{SiO}_{2}$ layer under the small structures, but maintain the majority of the $\mathrm{SiO}_{2}$ under the large pads and electrode leads. $\mathrm{The}_{\mathrm{SiO}}$ in these larger areas acted to electrically insulate the leads from one another and provide additional structural support for the free-standing microstructures. This wet etching procedure was a critical step to prevent exposing the cantilever beams to surface tension which caused them to irreversibly stick to the substrate, a phenomenon 
known as "stiction". For the wet etching procedure, a small Nalgene ${ }^{\circledR}$ beaker, not much larger than the SOI die substrate, was placed inside a much larger Nalgene ${ }^{\circledR}$ beaker. Subsequently, two pipettes filled with BOE were placed in the small beaker and emptied until the SOI die substrate was completely covered with BOE. The individual dies were etched with BOE for 30 minutes, after which the BOE was displaced with DI water.

The displacement was performed by gently spraying DI water into the small beaker and letting it overflow into the larger beaker. The fluid, diluted BOE, in the large and small beaker was removed using an aspirator while care was taken not to remove all the liquid in the small beaker. It was very important that the SOI die stayed completely submerged in the fluid to prevent stiction as mentioned earlier. This process was repeated a total of four times so that the remaining liquid in the small beaker was almost entirely DI water. The same displacement process was repeated with IPA four times until the liquid covering the SOI die was almost entirely IPA. At this point, the SOI die substrate was ready for critical point drying.

\section{Critical Point Drying Process}

After wet etching, critical point drying was used to dry the SOI die to prevent the surface tension of the drying IPA from deforming the free standing cantilever structure during the final nitrogen drying step. The critical point of a liquid-vapor system is the temperature and pressure at which a phase boundary ceases to exist, or there is no distinction between the liquid and the gas. Critical point drying was performed using the SAMDRI ${ }^{\circledR}$-PVT-3D system (Tousimis Research Corporation, Rockville, MD) and $\mathrm{CO}_{2}$ was used as the transitional medium, which has a critical point at $31.1^{\circ} \mathrm{C}$ and $1072 \mathrm{psi}$. The SOI die substrate was placed in the chamber, which was partially filled with IPA 
ahead of time, and sealed shut. When moving the SOI die from the IPA-filled beaker to the SAMDRI chamber, it was crucial to hold the die level to insure the IPA meniscus covered the cantilever beams. The chamber was then cooled below $0^{\circ} \mathrm{C}$ and filled with liquid $\mathrm{CO}_{2}$ until all IPA was purged from the system. Next, the chamber was heated to a temperature above the critical temperature of $\mathrm{CO}_{2}$, converting the liquid $\mathrm{CO}_{2}$ to the gaseous form. Finally, the gaseous $\mathrm{CO}_{2}$ was vented from the chamber at a temperature above $31.3^{\circ} \mathrm{C}$ and the chamber was allowed to return to room temperature and pressure. The finished device was removed from the chamber and prepared for testing and characterization.

\section{Piezoresistive Detector Characterization Studies}

Microcantilevers operate in either static or dynamic modes for sensing purposes. To determine the novelty and usefulness of an asymmetric microcantilever, the cantilever sensitivity must be tested in both static and dynamic situations. Static actuation of a microcantilever occurs when the beam is initially unloaded and not moving. The beam is then deformed by an external stimulus and remains in the deformed position until the external stimulus is removed. Thus, the piezoresistor has a constant application of stress that does not generate heat other than the Joule heating generated by the DC voltage across the piezoresistor. For these static piezoresistance tests, an electrically isolated needle statically actuated the piezoresistors. Dynamic loading of a microcantilever occurs while the microcantilever is in resonance, or vibrating at its natural frequency. The mass of the vibrating cantilever beam is altered by an external stimulus, changing the fundamental frequency of the vibrating beam. The resistance across the microcantilever base changes from its absolute maximum to its absolute minimum during one dynamic 
cycle. At high frequencies, this motion also generates heat through cyclic stress loading (ie. mechanical damping). Therefore, the dynamic and static sensitivity must be determined and evaluated separately to validate the usefulness of the asymmetric cantilever design.

\section{Determination of Static Piezoresistor Resistance}

Static resistance measurements were made of the various cantilever geometries to determine base-line resistances. The average resistance of the piezoresistors will be used to determine the appropriate resistor for use in the voltage dividing sensor circuit. The resistor should have a value equivalent to the piezoresistor value when the microcantilever is in a non-deflected state. A probe station (Microchamber Attoguard, Cascade Microtech, Beaverton, OR) was used to perform the static characterization studies on the microcantilever piezoresistors (Figure 13). The probe station consisted of two probes which were placed in contact with the bond pads electrically connected across the piezoresistors. The probes were connected to a precision semiconductor parameter analyzer (Model Agilent 4156C, Agilent Technologies Inc., Santa Clara, CA) that passed a fixed current $(600 \mu \mathrm{A})$ through the piezoresistor while monitoring the output voltage. A graph of the voltage versus current was produced for each microcantilever beam (Figure 14). The relationship between voltage and current was linear for these piezoresistors, so a representative point was chosen on the graph for a voltage value and the corresponding current value. The output voltage was divided by the current to give a resistance value in accordance with Ohm's Law:

$$
R=V / I
$$




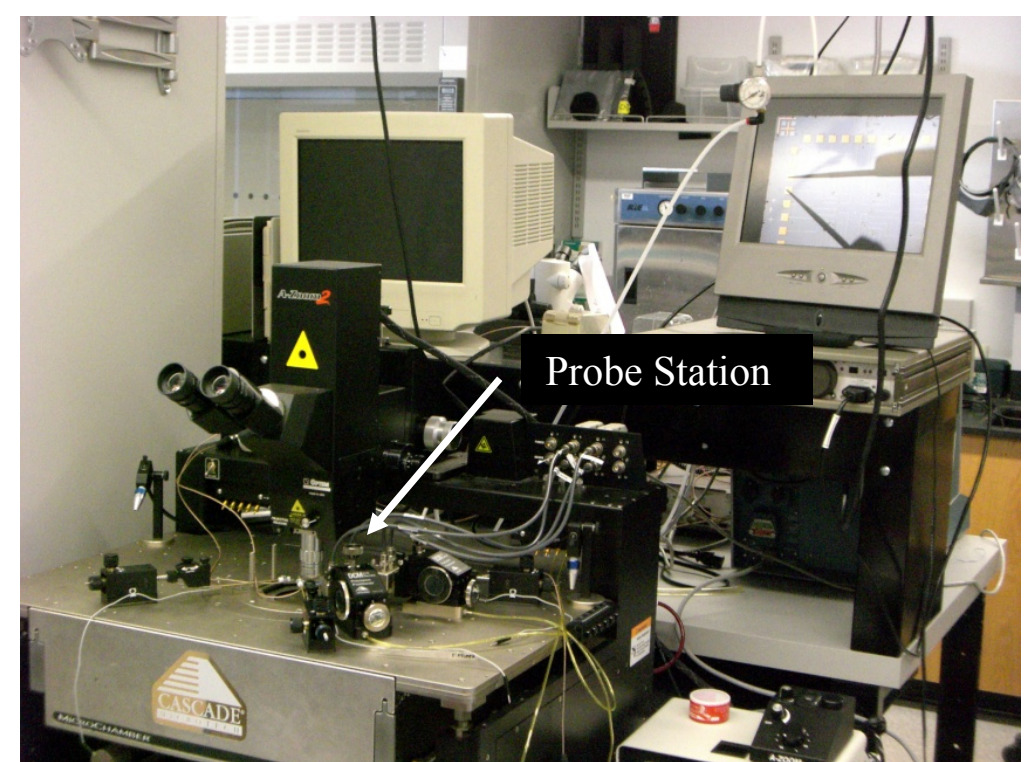

Figure 13 - Probe Station Used to Measure Resistance Across Piezoresistors.

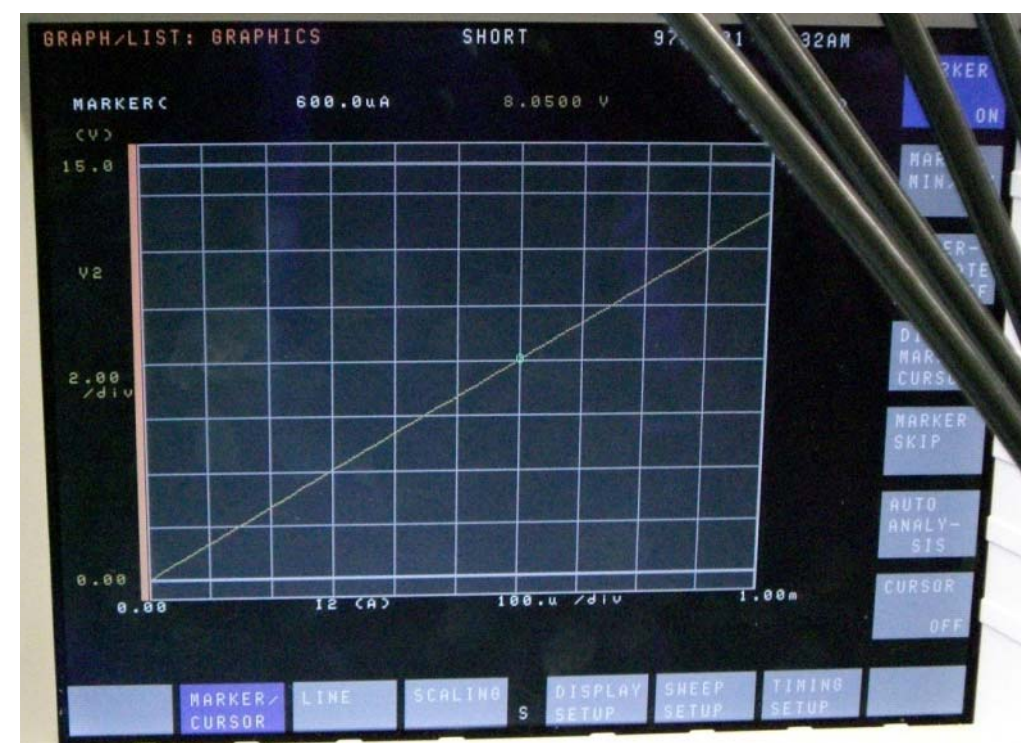

Figure 14 - Typical Graph of Voltage Versus Current Across a Piezoresistor.

2. Microcantilever Metrology

Each microcantilever's geometry was carefully measured in an SEM (Zeiss- LEO 1430, Carl Zeiss SMT AG, Germany) using built-in software tools and recorded.

Specifically, the piezoresistor width, leg length, offset, and beam length (see Figure 15 as a reference for these terms) were measured. The cumulative mean and standard deviation 
for each geometrical design for all die were calculated to compare the fabricated results to the original design.

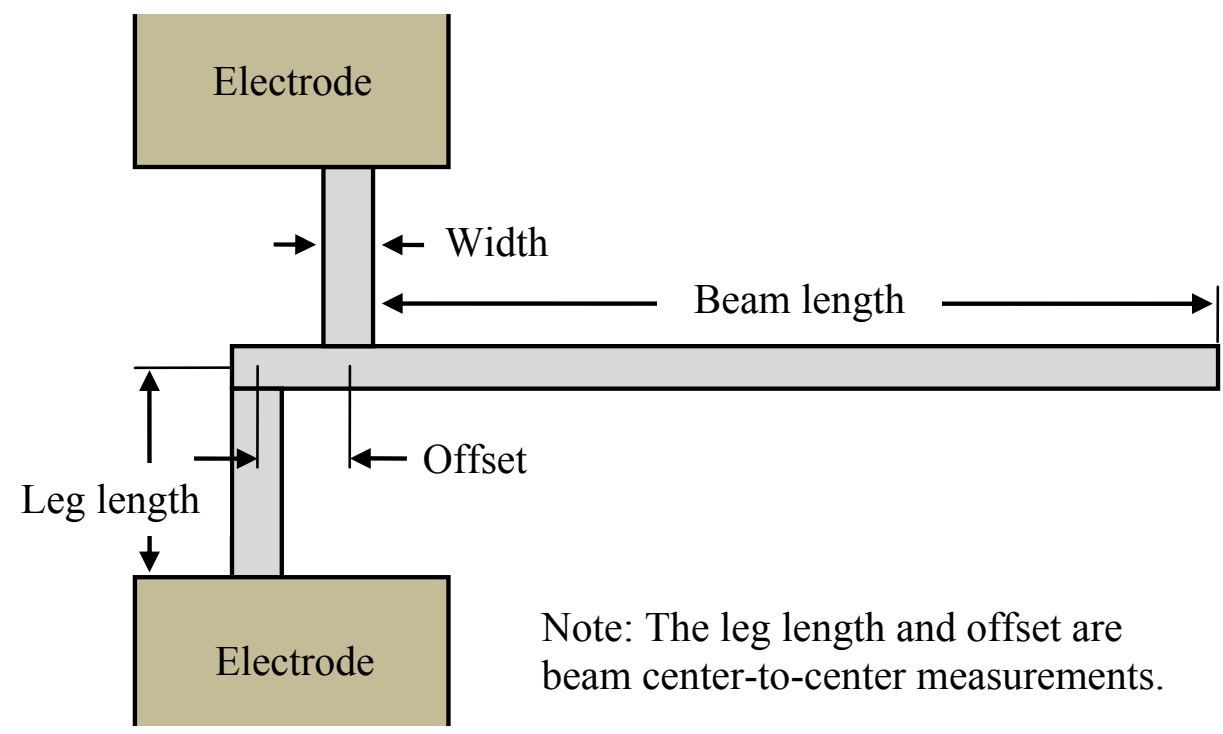

Figure 15 - Microcantilever Geometry Measurements.

D. Resonator and Sensitivity Detection Experiments

1. Resonator Actuation Experiments

An experiment was performed to determine whether the resonators could vibrate laterally with an AC driving signal, and to find each microcantilever's resonant frequency and threshold voltage. The device dies were loaded into the sealed chamber of a cryogenic probe station (Test Equipment Solutions LTd, Berkshire, UK) and the pressure was reduced to about $1 \times 10^{-7}$ Torr. Tungsten test probes (PTT-120/4-25, $45 \mathrm{deg}, 12 \mu \mathrm{m}$, Cascade Microtech, Beaverton, OR) were used to make electrical contact with electrode A, B, and C of each microcantilever (Figure 16). A function generator (Agilent 33220A, Agilent Technologies Inc., Santa Clara, CA) provided the AC bias to electrode A and simultaneously sent a reference signal to the lock-in amplifier (EG\&G 5210, Test Equipment Solutions Ltd, Berkshire, UK). The function generator served as an AC signal 
source that provided a 10 Volt peak-to-peak, sinusoidal signal to the driving electrode which was swept from 0 to $80 \mathrm{kHz}$ in increments of $1 \mathrm{~Hz}$. A DC power supply (Agilent E3645A, Agilent Technologies Inc., Santa Clara, CA) supplied a 10 Volt DC bias across the piezoresistor and a fixed resistor $(14 \mathrm{k} \Omega)$, forming a simple voltage divider circuit. The fixed resistor value matched the mean resistance value of the piezoresistors calculated in a previous sub-section. The input to the lock-in amplifier was connected to electrode B and the output was read by a digital multimeter (Keithley 196, Keithley Instruments Inc., Cleveland, $\mathrm{OH})$. Electrode $\mathrm{C}$ was connected to universal ground. The lock-in amplifier was used for phase-sensitive determination of the AC electrical potential between the fixed resistor and the piezoresistor. The function generator, DC power supply, and digital multimeter were connected to a PC using GPIB cables interfaced to a LabVIEW program that served as the data acquisition system (Figure 17 \& Figure 18).



Figure 16 - Electrode Layout for Microcantilever Device. 


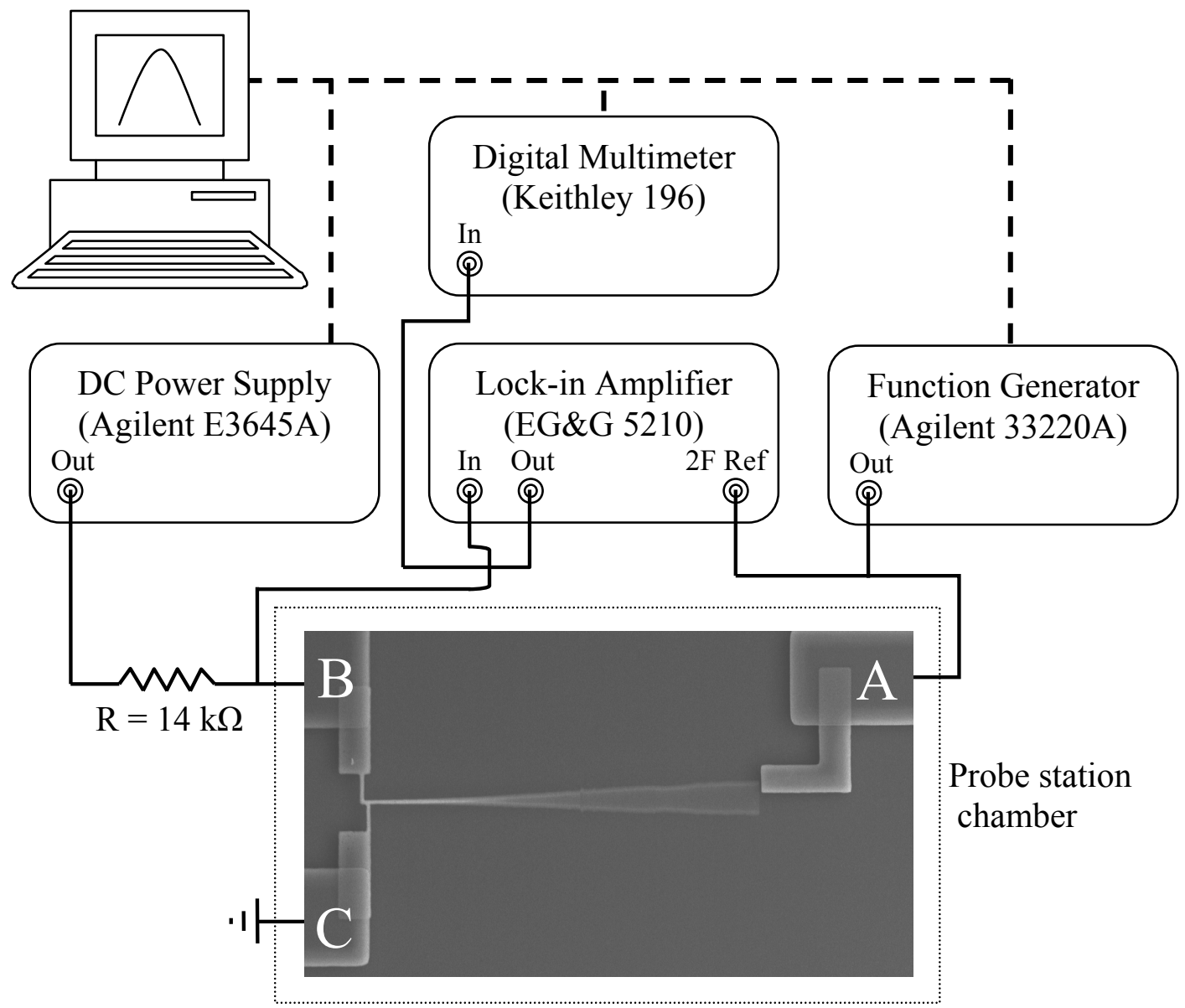

Figure 17 - Schematic of General Instrumentation Setup. (-) BNC Cable, (- - - ) GPIB Cable.
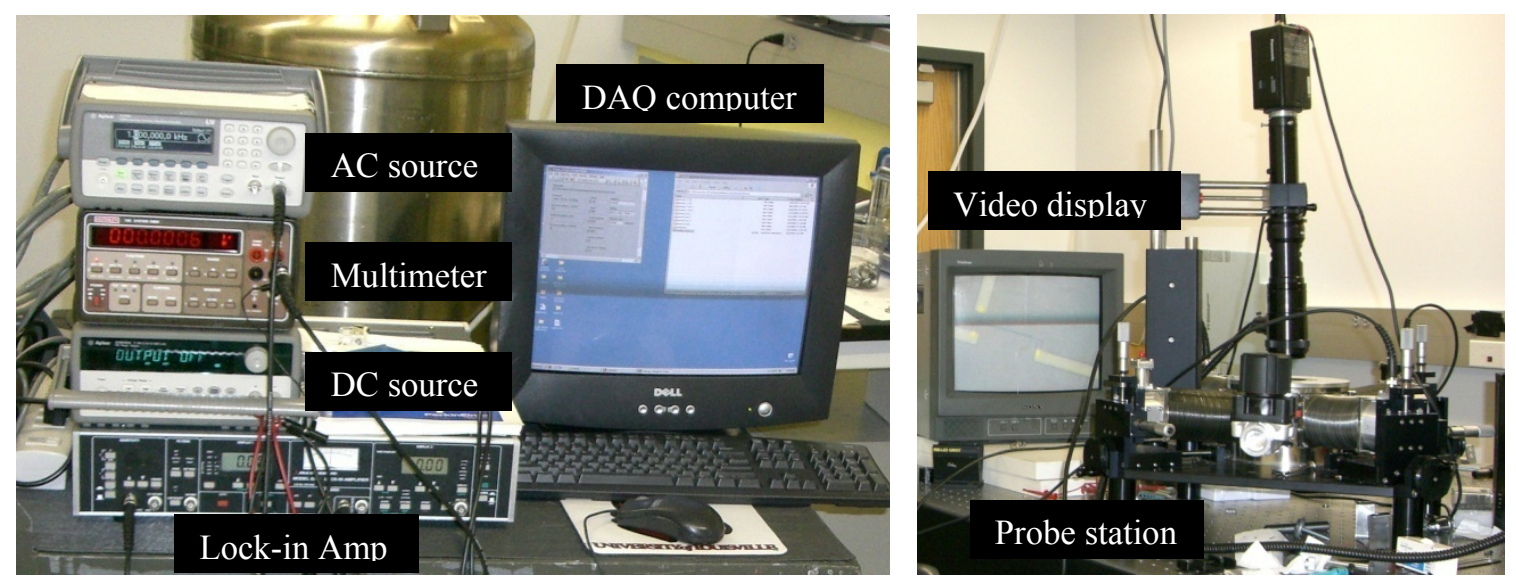

Figure 18 - Instruments Used in the Experimental Setup.

Lateral vibration was visually observed in the probe station using a video camera focused through a viewing port of the probe station. The driving frequency was typically 
swept from $0 \mathrm{kHz}$ to $80 \mathrm{kHz}$ at a rate of $1 \mathrm{~Hz}$ per second. After the frequency range was swept, the output data was plotted in an Excel® graph as frequency versus voltage output from the lock-in amplifier and the data was inspected for resonance frequency peaks. A LabVIEW virtual instrument algorithm was custom-designed and developed (see Appendix A) and a front panel was displayed on the monitor of a PC (Pentium 4 CPU 2.80 GHz, 512 MB Ram, Dimension 3000, Dell Inc., Round Rock, TX) during testing (Figure 19).

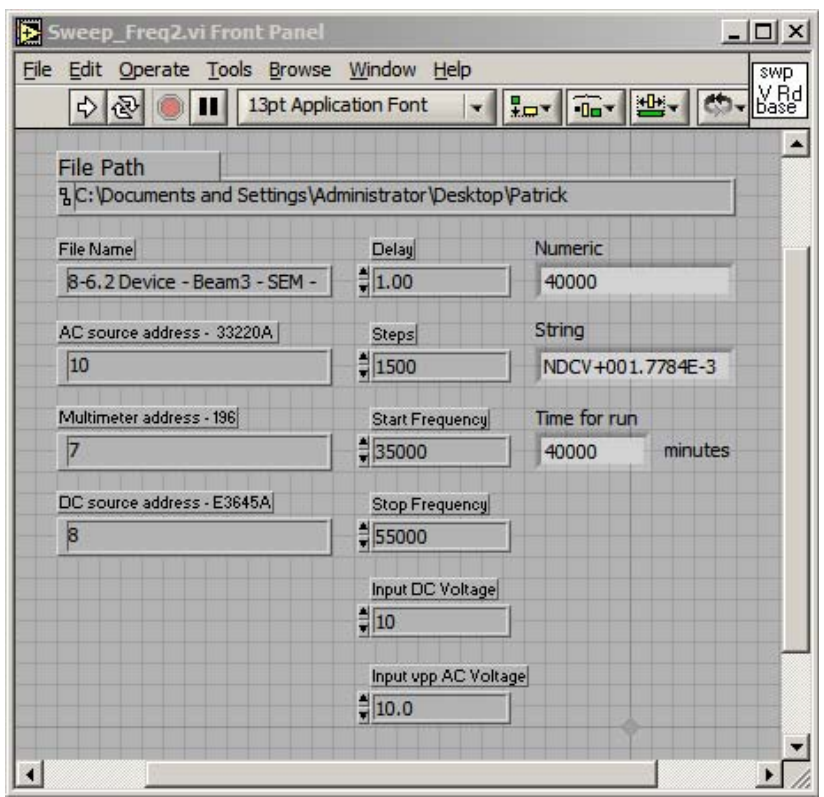

Figure 19 - LabVIEW VI Entitled Sweep_Freq2.Vi Used To Sweep The Resonant Frequencies.

As the drive frequency neared the resonant frequency of the cantilever, the cantilever tip would begin to vibrate. The resonant frequency of each microcantilever was verified when the output of the lock-in amplifier reached a peak value which was demonstrated by looking for a voltage output peak on the Excel ${ }^{\circledR}$ graph of frequency sweep data for lock-in amplifier voltage output versus frequency. The resonant frequency was also confirmed when the cantilever tip showed maximum lateral displacement. This maximum lateral displacement could only be observed visually on the video monitor but 
was not measured (see an example in Figure 20). The cantilever tip blurred and swung rapidly from side-to-side only when it was in resonance and at no other time during the frequency sweep. The resonant frequency was recorded for each cantilever beam and the lock-in amplifier output for the swept frequency was saved to an Excel ${ }^{\circledR}$ data file. Each microcantilever on the seven completed die packages was checked in the frequency range $0 \mathrm{kHz}$ to $80 \mathrm{kHz}$ for resonance.

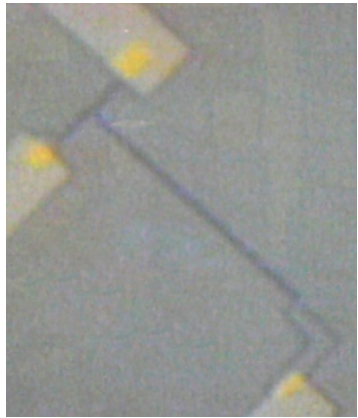

(a)

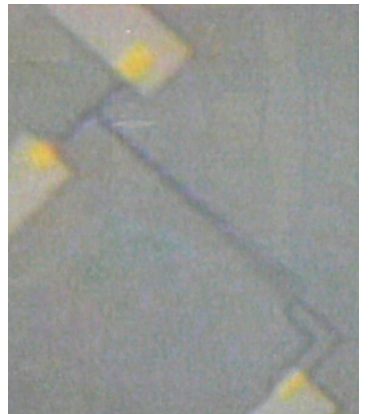

(b)

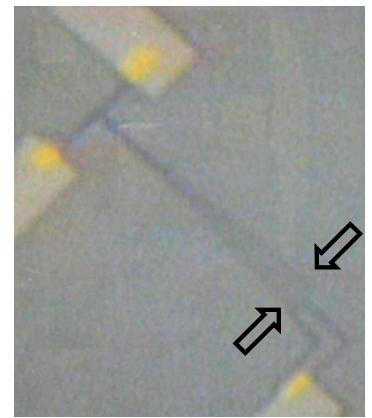

(c)

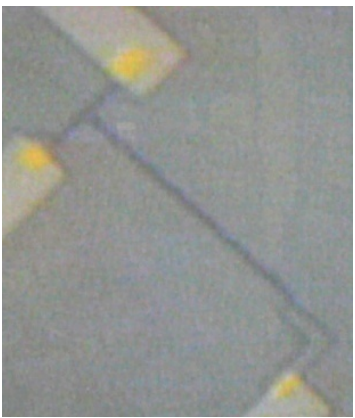

(d)

Figure 20 - A Beam5 Microcantilever Resonating In The Probe Station.

\section{Static Deflection Tests}

To determine whether the new piezoresistor geometry increased the sensitivity of the microcantilevers, static deflection tests were performed to determine the correlation between tip deflection and piezoresistance. Device dies were placed in an SEM (Zeiss Supra 35VP, Carl Zeiss SMT AG, Germany) and a digital multimeter (Agilent 34410A) was connected to electrodes B and C (see Figure 16). A tungsten needle actuated by a nanomanipulator (Zyvex S100 Nanomanipulator, Zyvex Instruments, Richardson, TX) was used to deflect the tip of the microcantilever in $4 \mu \mathrm{m}$ increments governed by an overlaid grid. The deflecting probe was gently lowered near the tip of the microcantilever until it made contact with the silicon substrate. The probe was then raised until it no longer made contact with the substrate, but was on a horizontal level with the suspended cantilever beam. The probe was slowly moved into contact with the cantilever tip and the 
tip was deflected in $4 \mu \mathrm{m}$ increments. At each measurement location, the probe was stopped and the resistance of the piezoresistor was allowed to stabilize.

Resistance measurements were recorded at each lateral deflection distance, which was normally a total distance of $20 \mu \mathrm{m}$, or 5 measurements. Displacement versus resistance was plotted for each microcantilever and the sensitivity of the device was the slope this line. The static sensitivity was calculated as

$$
\text { Static Sensitivity }=\frac{R_{1}-R_{0}}{\Delta x}
$$

where $\mathrm{R}_{0}$ is the beginning resistance at zero deflection and $\mathrm{R}_{1}$ is the maximum resistance at a deflection of $\Delta \mathrm{x}$. Static sensitivity is given in $\Omega / \mu \mathrm{m}$. The probe was then moved in the opposite direction until the cantilever tip was released and freely suspended. Videos were captured of each static deflection test and an overall view of the probe setup is shown in Figure 21. The nanomanipulators and a microcantilever device array are shown in Figure 22.

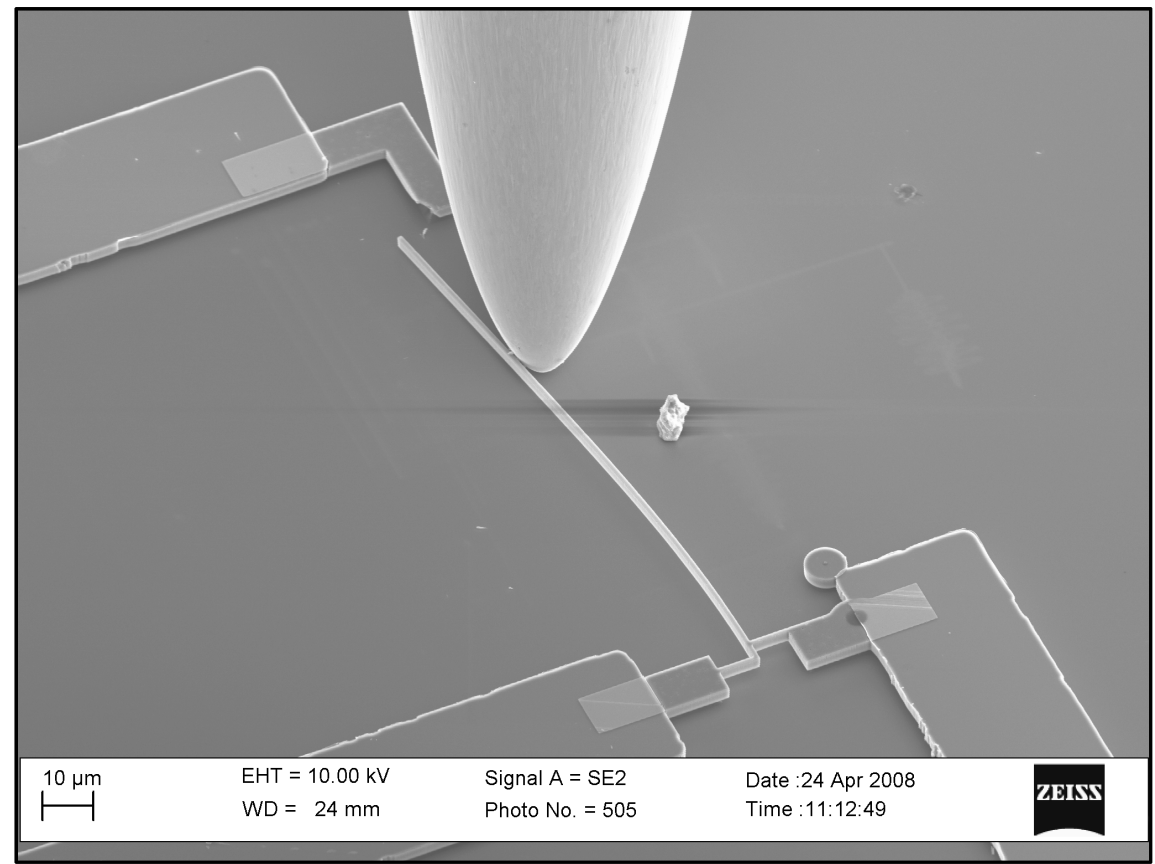

Figure 21 - Tungsten Needle Statically Actuating a Microcantilever in an SEM. 


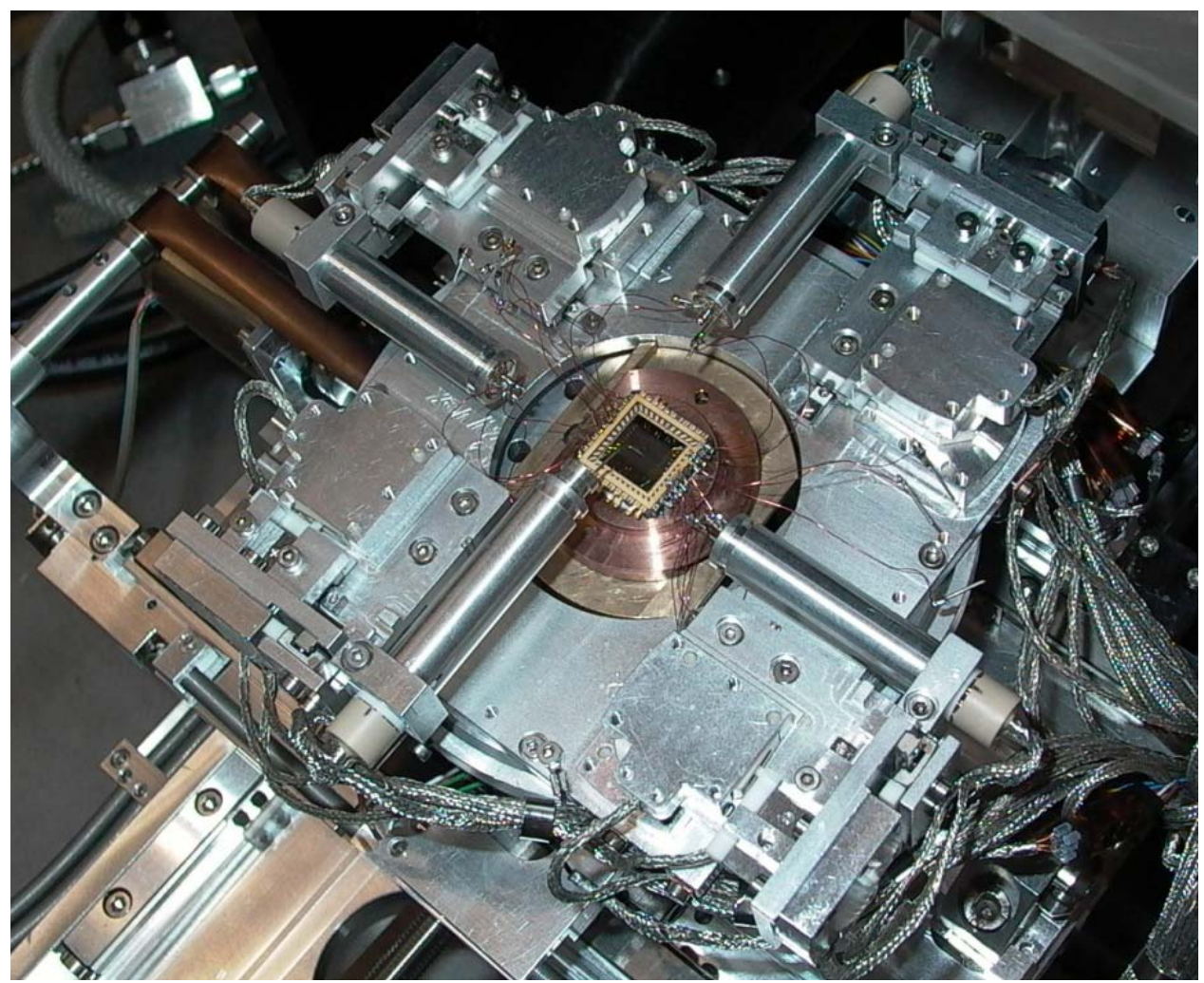

Figure 22 - Nanomanipulators and Microcantilever Device in an SEM.

3. Dynamic Deflection Tests

Sensitivity tests were also performed on resonating microcantilevers to determine the correlation between tip deflection and piezoresistance during resonance. Device dies were placed in an SEM (Zeiss LEO 1430) and wired into the instrumentation circuit shown in Figure 17 using a leaded chip carrier (CCJ04419, Spectrum Semiconductor Materials, Inc., San Jose, CA) and soldered to a custom-made prototyping circuit board (Figure 23). The microcantilever to be tested was brought into resonance using the frequency value already determined in the probe station. The resonant frequency was locked in when the cantilever tip appeared to be at its maximum lateral vibration and an image of the cantilever was taken; the total tip displacement, $\mathrm{V}$, was measured using software tools and the captured image (see Figure 24 for reference). As shown in Figure 24, when the cantilever was static (solid line) the width of the cantilever equaled W; 
however, when the cantilever was vibrating (dashed line) the width of the blurred tip equaled $\mathrm{V}$. The deflection of the cantilever tip, $\Delta \mathrm{x}$, was calculated as

$$
\Delta x=\frac{V-W}{2} .
$$
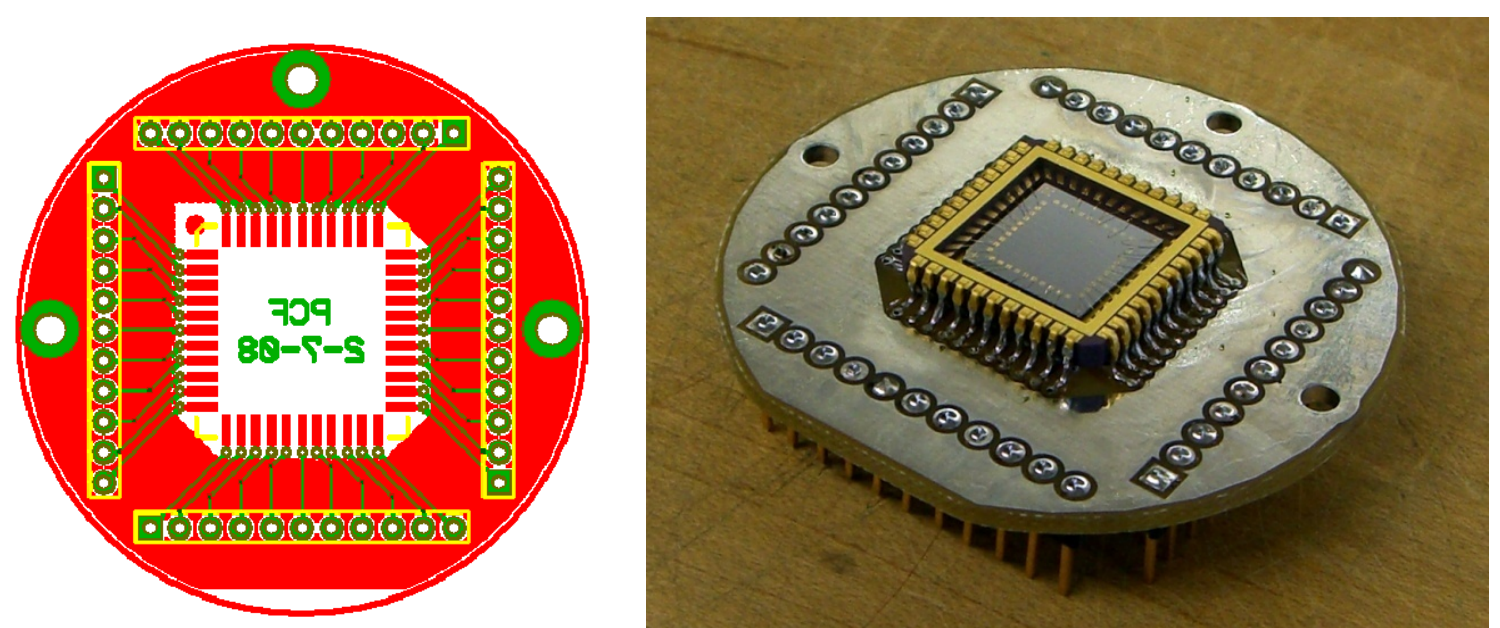

Figure 23 - Custom-Made Prototyping Circuit Board for Imaging Microcantilever Array in an SEM.

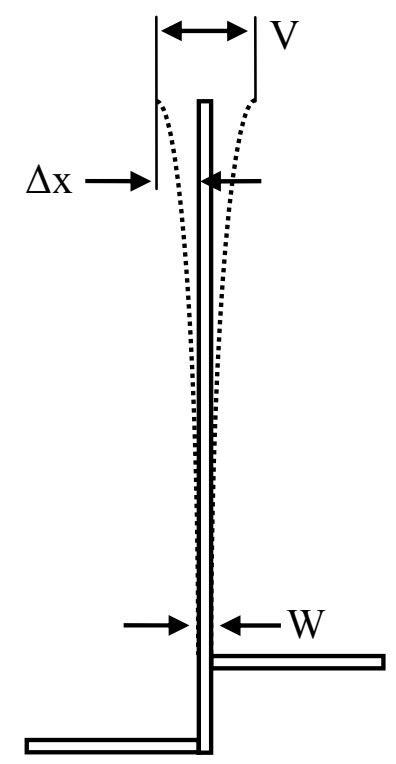

Figure 24 - Schematic Drawing of the Resonating Cantilever Deflection Measurement.

The cantilever drive frequency was changed to a value that reflected absolutely no movement in the cantilever tip; the drive frequency was changed to the resonant frequency minus $700 \mathrm{~Hz}$. A frequency range of $1 \mathrm{kHz}$ was then swept using 
Sweep_Freq2.vi with the resonant frequency occurring about two-thirds of the way through the sweep. The cantilever movement quickly falls off after the resonance frequency is reached, so this $1 \mathrm{kHz}$ range effectively provides data on the piezoresistance of the dynamic cantilever before resonance, during peak resonance, and after resonance. The output of the lock-in amplifier during the sweep was saved to an Excel ${ }^{\circledR}$ data file. Then, the digital multimeter output for resonance and non-resonance was converted to a voltage at node B, shown in Figure 25. The lock-in amplifier multiplies the sensed voltage at node B by a gain value according to the sensitivity setting on the lock-in amplifier. The gain for each dynamic sensitivity test was calibrated so that at resonance, the output of the lock-in amplifier was between 5 and 8 Volts. Therefore, the gain values used for each test varied and a conversion chart (shown in Table II) was used to convert the lock-in amplifier voltage output to the actual output at node B in Figure 25. Equation 3 was used to calculate the current from node A to B as well as the resistance of the piezoresistor for resonance and non-resonance together with the voltages at node $\mathrm{B}$. The dynamic sensitivity was calculated as:

$$
\text { Dynamic Sensitivity }=\frac{R_{1}-R_{0}}{\Delta x} \quad(\Omega / \mu \mathrm{m})
$$

where $R_{0}$ is the calculated resistance at non-resonance, $R_{1}$ is the calculated resistance at resonance, and $\Delta \mathrm{x}$ is the measured lateral displacement of the microcantilever at resonance. 
To Lock-in

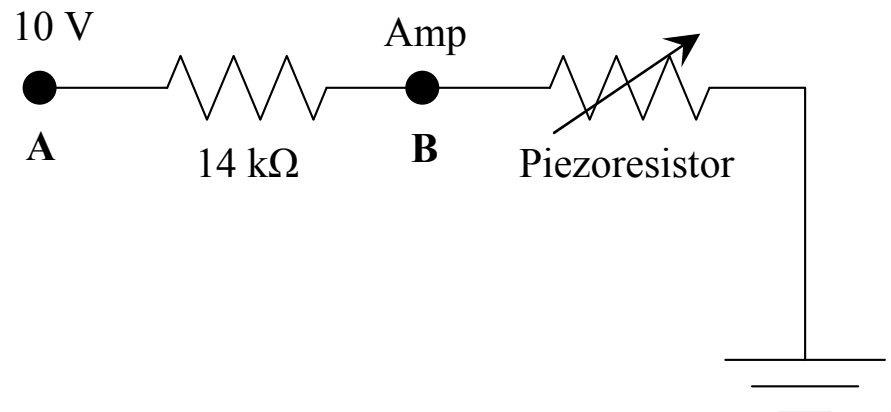

Figure 25 - Schematic of Voltage Divider Circuitry.

TABLE II

LOCK-IN AMPLIFIER GAIN AT SELECTED SENSITIVITY VALUES

\begin{tabular}{|c|c|}
\hline $\begin{array}{c}\text { Full-scale } \\
\text { sensitivity }\end{array}$ & $\begin{array}{c}\text { Gain at dynamic } \\
\text { reserve setting }\end{array}$ \\
\hline $3 \mathrm{~V}$ & 0.3332 \\
\hline $1 \mathrm{~V}$ & 1 \\
\hline $300 \mathrm{mV}$ & 3.332 \\
\hline $100 \mathrm{mV}$ & 10 \\
\hline $30 \mathrm{mV}$ & 3.332 \\
\hline $10 \mathrm{mV}$ & 10 \\
\hline $3 \mathrm{mV}$ & 33.32 \\
\hline $1 \mathrm{mV}$ & 100 \\
\hline
\end{tabular}




\section{IV.RESULTS}

Fabrication methods were refined and used to create laterally vibrating microresonators with arbitrary geometries. Seven microcantilever devices were successfully fabricated, consisting of ten microcantilevers each, for a total of seventy microcantilevers. Not all microcantilevers were successfully tested due to fabrication and cleanliness issues. The actuation of these microcantilevers was verified using Scanning Electron Microscopy. The piezoresistive detector was characterized by measuring the static and dynamic sensitivities of these resonators. The details of the results from these studies are described below.

\section{A. Determination of Static Piezoresistor Resistance}

The mean static piezoresistance of the microcantilevers was determined by measuring the resistance of the microcantilevers in one array. The resistance measurements for a typical device are shown in Table III.

TABLE III

\section{CALCULATED RESISTANCE FOR DEVICE 1}

\begin{tabular}{|c|c|c|c|c|c|}
\hline Beam & $V$ (Volts) $=$ & $\mathbf{I}(\boldsymbol{\mu A}) *$ & $\mathrm{R}(\mathrm{k} \Omega)$ & PL $(\mu \mathrm{m})$ & $\mathbf{P W}(\mu \mathrm{m})$ \\
\hline 1 & 8.7282 & 600.0 & 14.547 & 9 & 1.1 \\
\hline 2 & 8.0500 & 600.0 & 13.417 & 41 & 1.1 \\
\hline 3 & 7.254 & 600.0 & 12.090 & 41 & 1.1 \\
\hline 4 & 8.4810 & 600.0 & 14.135 & 41 & 1.1 \\
\hline 5 & 10.1894 & 600.0 & 16.982 & 41 & 1.3 \\
\hline 6 & 9.988 & 600.0 & 16.647 & 41 & 1.1 \\
\hline 7 & 8.6430 & 600.0 & 14.405 & 41 & 1.1 \\
\hline 8 & 7.2836 & 600.0 & 12.139 & 21 & 1.1 \\
\hline 9 & 8.565 & 600.0 & 14.275 & 21 & 1.1 \\
\hline 10 & 10.5606 & 600.0 & 17.601 & 21 & 1.1 \\
\hline Average & & & 14.624 & & \\
\hline
\end{tabular}




\section{B. Microcantilever Metrology}

The metrology measurements for all seven device array dies are given in appendix B. Graphs of the mean leg length, leg separation, beam length, and beam width for each beam design across all the fabricated devices are shown in Figure 26, Figure 27, Figure 28, and Figure 29, respectively. Graphs of the percent error of fabrication parameters for each beam design across all the fabricated devices are shown in Figure 30, Figure 31, Figure 32, and Figure 33. The error bars represent the first standard deviation of the set of values. Percent error is defined as

$$
\text { Percent Error }=\frac{\text { Measured Value-Desired Value }}{\text { Desired Value }} * 100 .
$$

The mean absolute value of the percent error for the four geometry parameters is shown in Table IV, along with the mean percent error for the entire fabrication process (an average of the percent errors for each parameter). The beam width percent error was relatively high (12.90\%), as was the leg length percent error (9.24\%). The offset and beam length had much lower error, $5.94 \%$ and $4.85 \%$, respectively. The overall fabrication process had an $8.23 \%$ error. The percent error and standard deviation were much lower for parameters with larger dimensions; conversely it was much harder to produce small features with accuracy 


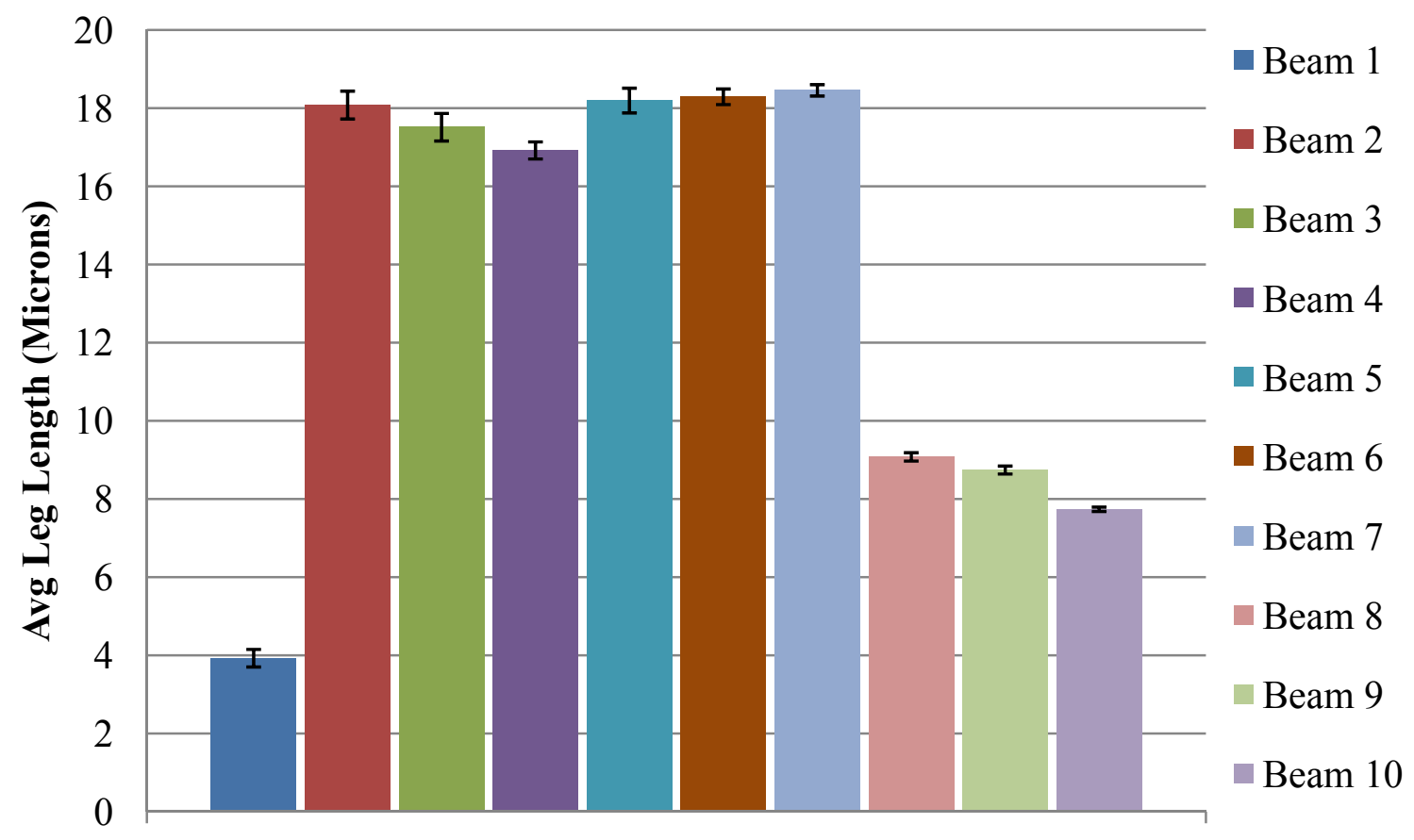

Figure 26 - Mean Leg Length and Standard Deviation for Measured Microcantilevers.

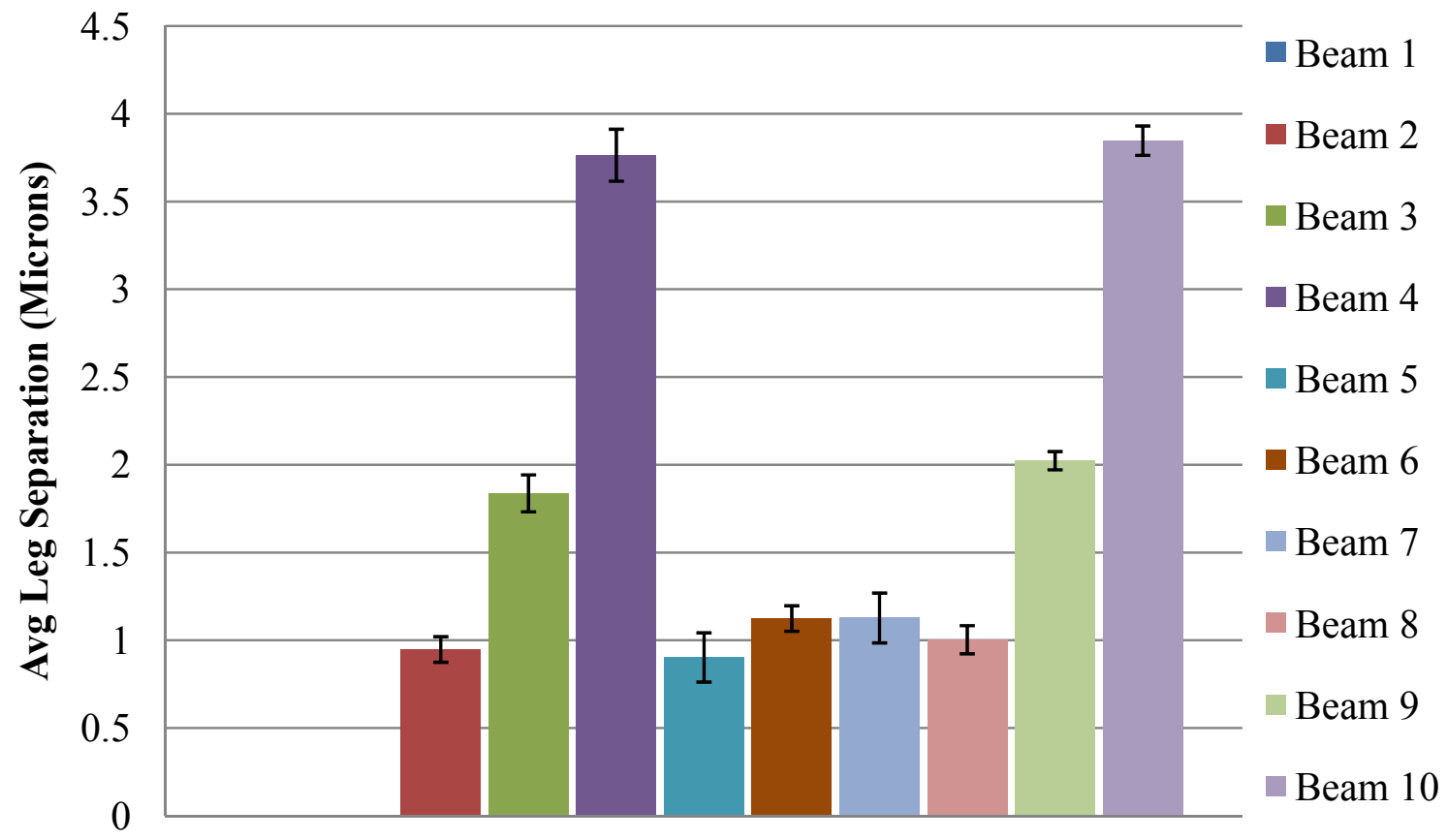

Figure 27 - Mean Leg Separation and Standard Deviation for Measured Microcantilevers. 


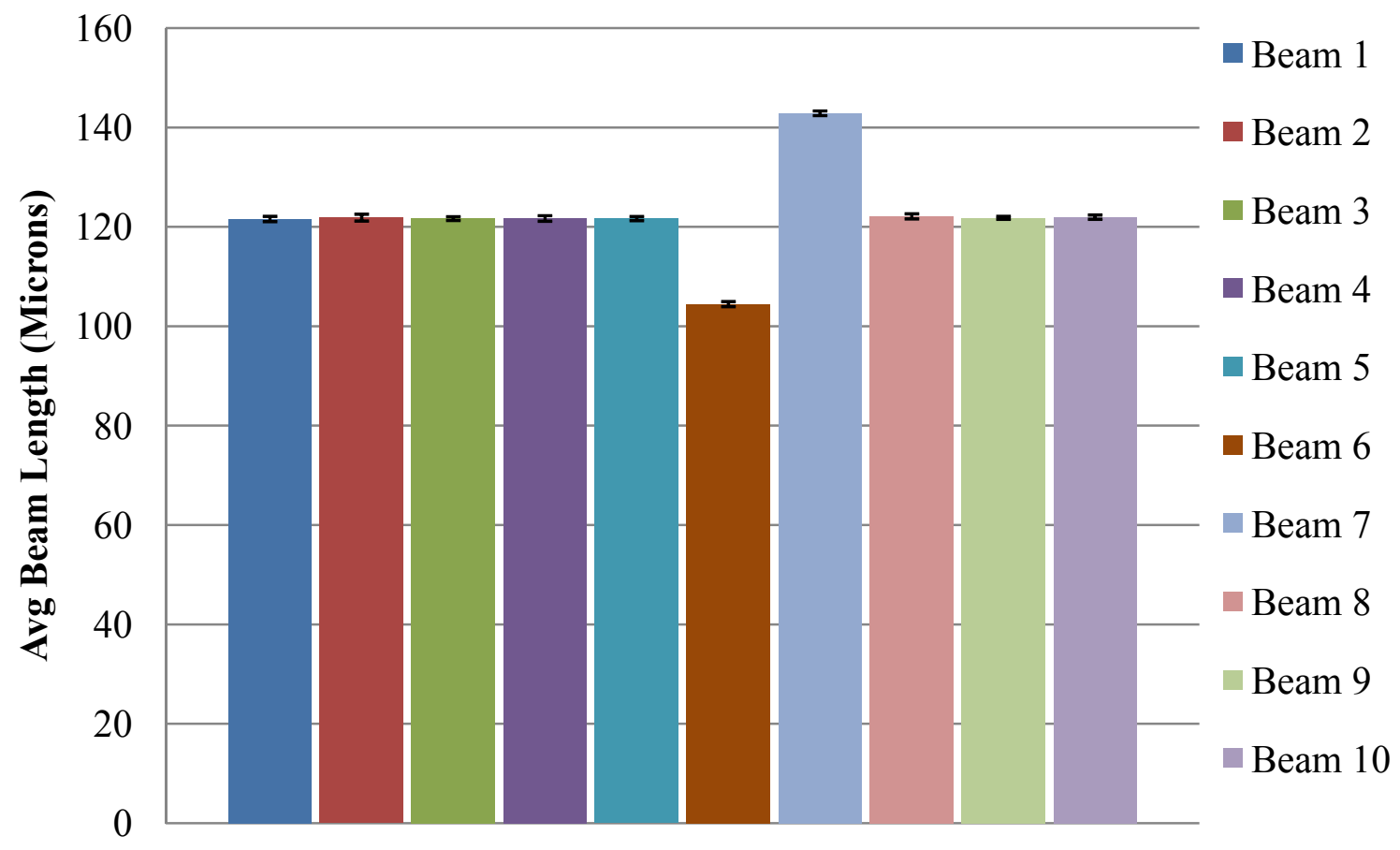

Figure 28 - Mean Beam Length and Standard Deviation for Measured Microcantilevers.

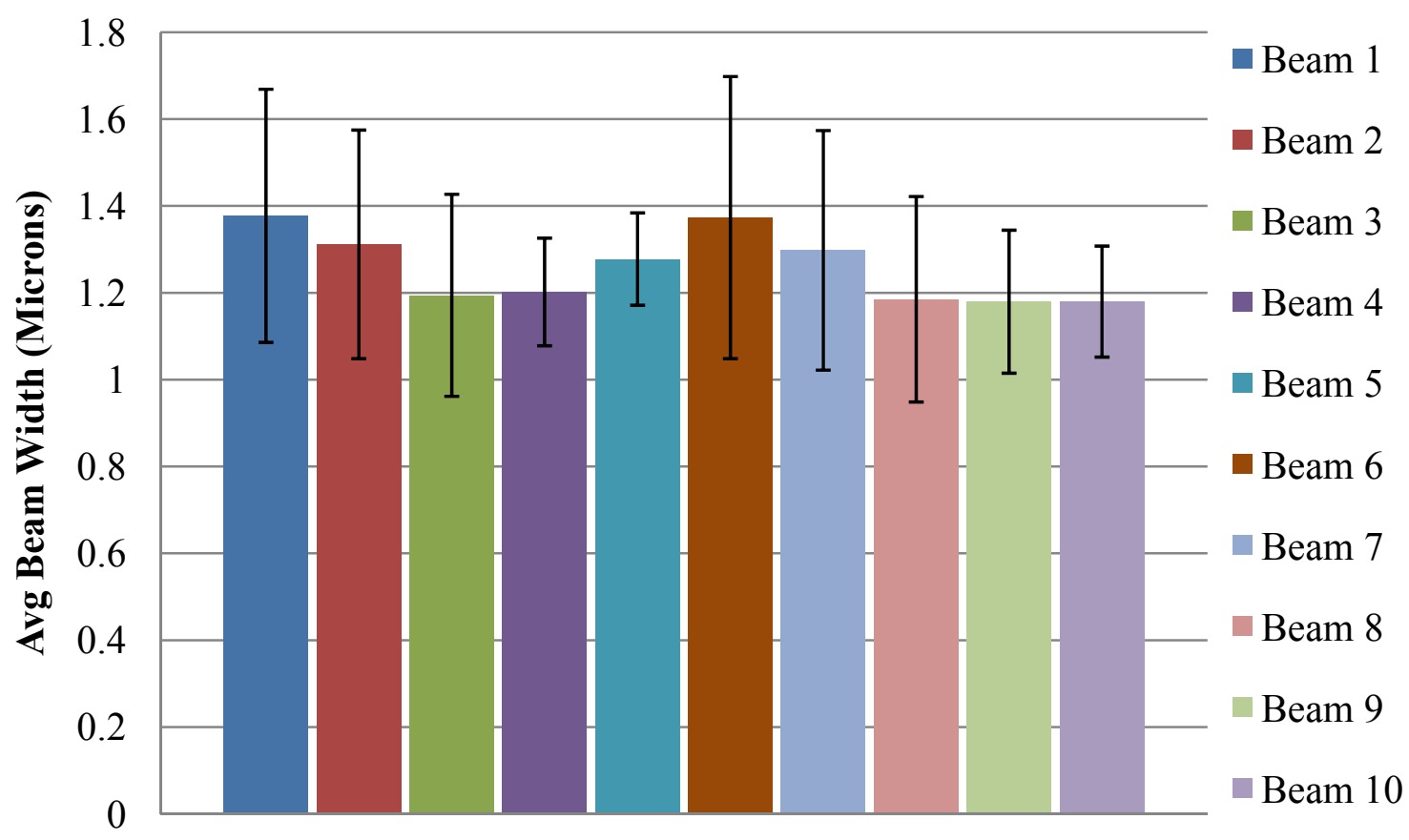

Figure 29 - Mean Beam Width and Standard Deviation for Measured Microcantilevers. 


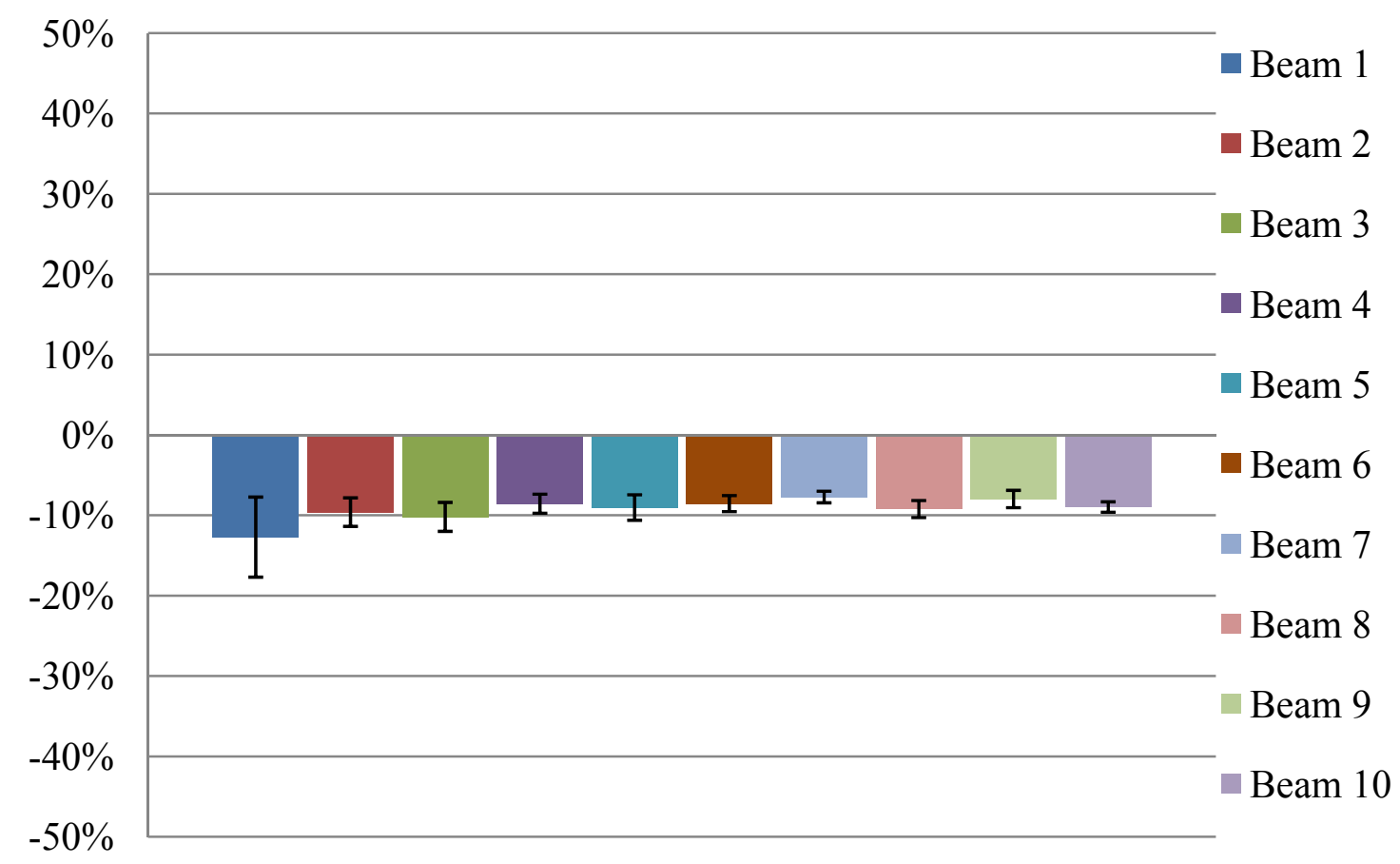

Figure 30 - Percent Error in Leg Length Parameter Fabrication.

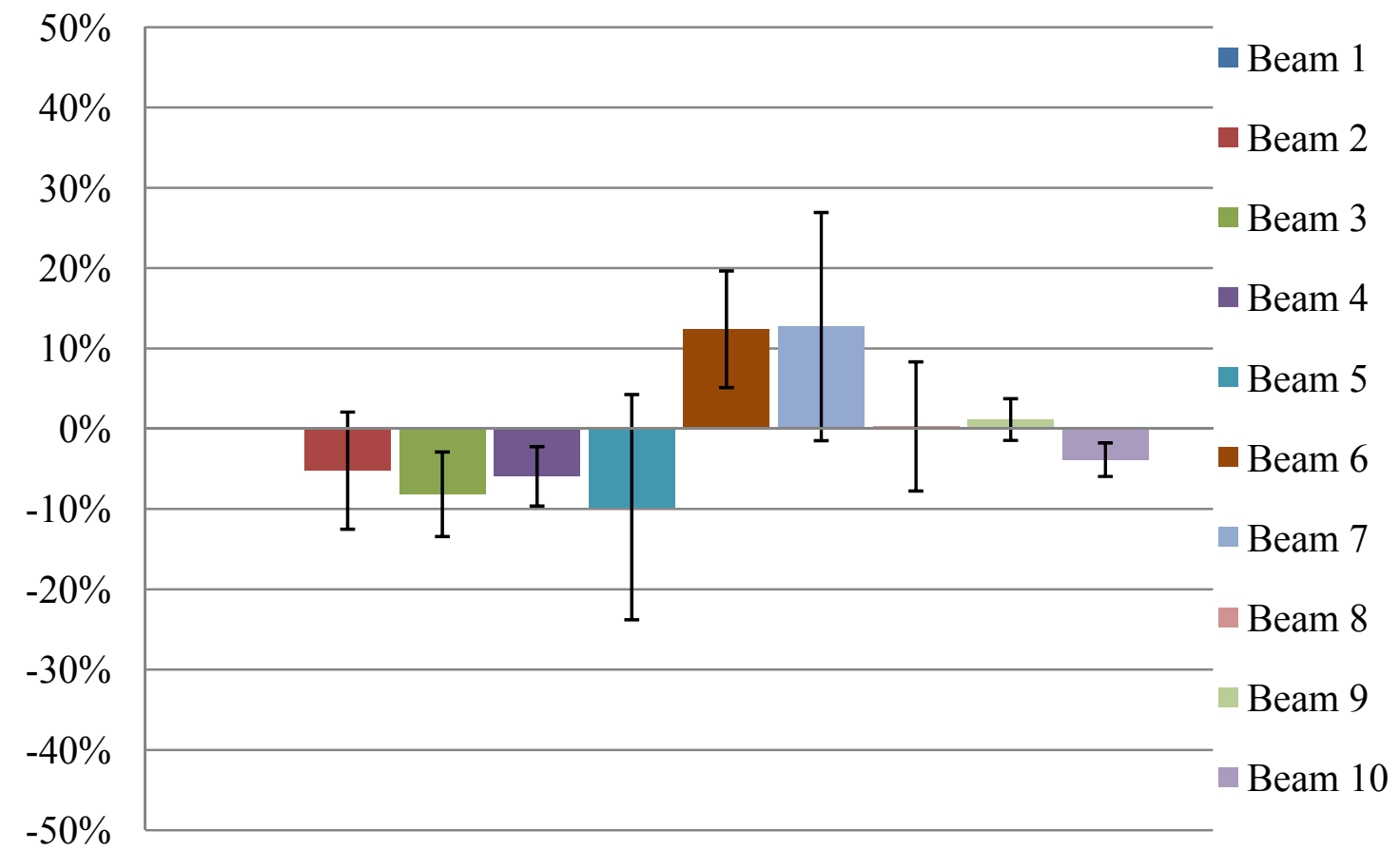

Figure 31 - Percent Error in Leg Separation Parameter Fabrication. 


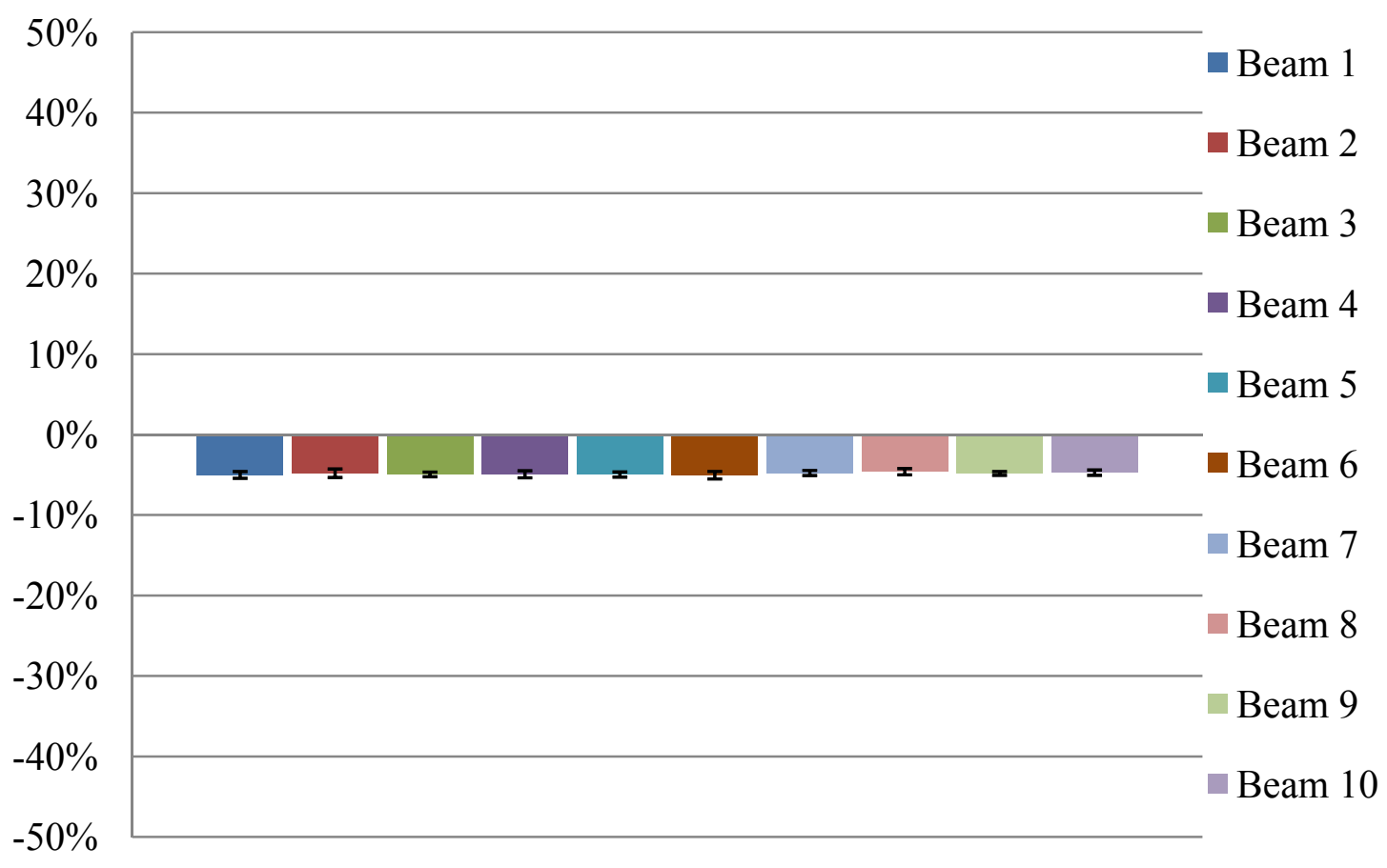

Figure 32 - Percent Error in Beam Length Parameter Fabrication.

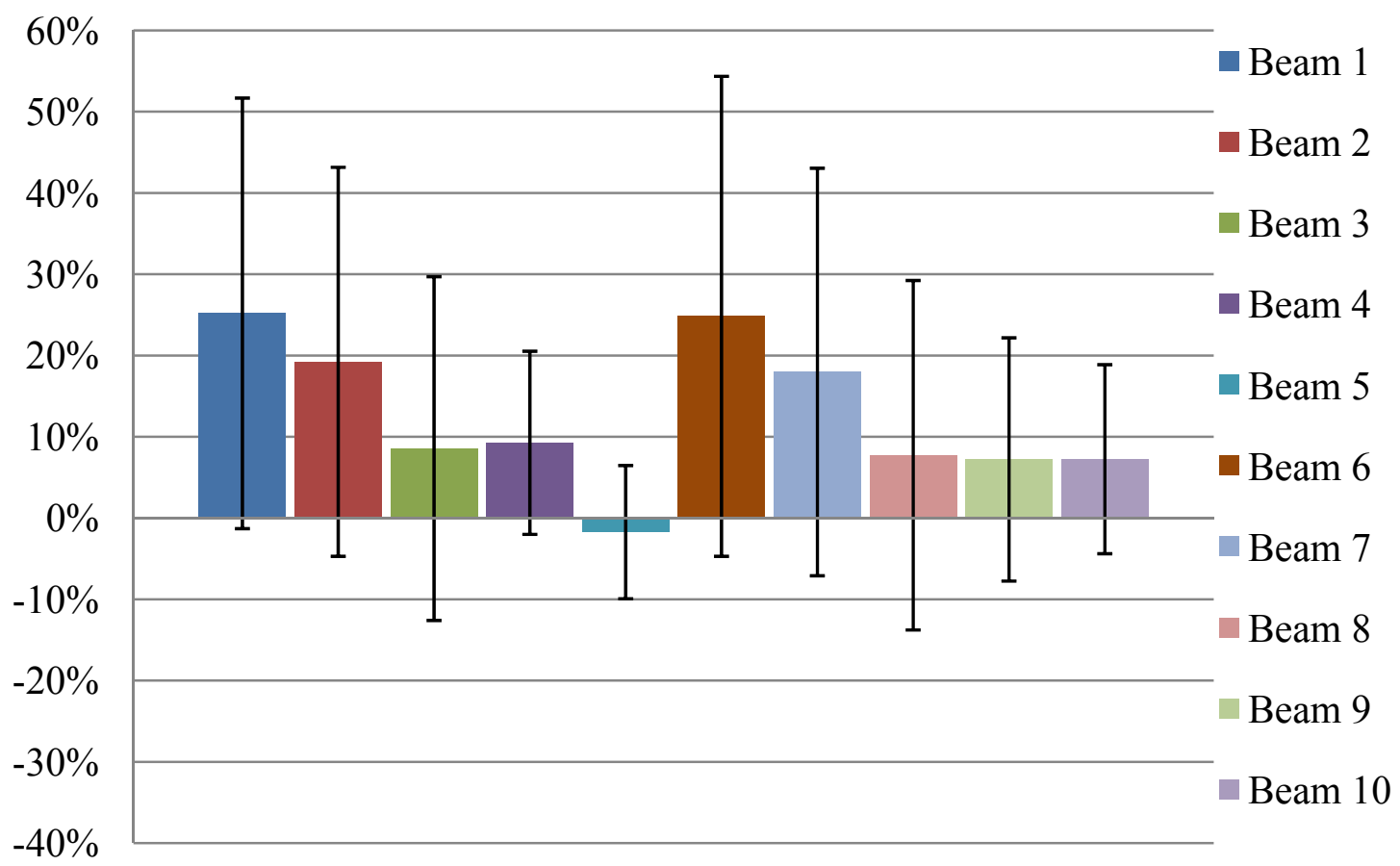

Figure 33 - Percent Error in Beam Width Parameter Fabrication. 


\section{TABLE IV}

\section{PERCENT ERROR FOR GEOMETRY PARAMETERS AND OVERALL ERROR}

\begin{tabular}{|c|c|}
\hline Parameter & Percent Error \\
\hline Beam Width & $12.90 \%$ \\
\hline Leg Length & $9.24 \%$ \\
\hline Offset & $5.94 \%$ \\
\hline Beam Length & $4.85 \%$ \\
\hline Mean Overall Error & $8.23 \%$ \\
\hline
\end{tabular}

C. Resonator Actuation Experiments

All microcantilever devices were tested in a vacuum-sealed probe station to determine their resonant frequency. The theoretical resonant frequency for each cantilever was found using Equations 8 and 9, where the Young's modulus of crystal silicon is $150 \times 10^{6} \mathrm{~g} /\left(\mu \mathrm{m} \bullet \mathrm{s}^{2}\right)$ and the density is $2.33 \times 10^{-12} \mathrm{~g} / \mu \mathrm{m}^{3}$. The results are shown in appendix B and a graph of the measured versus the theoretical resonant frequency is shown in Figure 34 . The calculated theoretical resonant frequency is always a factor of two larger than the measured resonant frequency because one full cycle of motion in the theoretical model constitutes the tip of the microcantilever passing the driving electrode twice. Thus, the theoretical frequency in Figure 34 has been divided by two for accurate comparison.

The resonant frequencies for microcantilever geometry designs 1 through 5 are between the range of $46,500 \mathrm{~Hz}$ to $57,500 \mathrm{~Hz}$ with no outliers. Microcantilever geometry design 6 has a relatively high mean resonant frequency compared to the other cantilevers, and geometry design 7 has a relatively low mean resonant frequency compared to the other cantilevers. This was expected because design 6 has a shortened cantilever (110 $\mu \mathrm{m})$ and design 7 has a lengthened cantilever $(150 \mu \mathrm{m})$. Microcantilever geometry 
designs 8 through 10 have resonant frequencies in the range of $40,000 \mathrm{~Hz}$ to $52,000 \mathrm{~Hz}$ with no apparent outliers. This range of frequency values is lower than the range for geometry designs 1 through 5 .

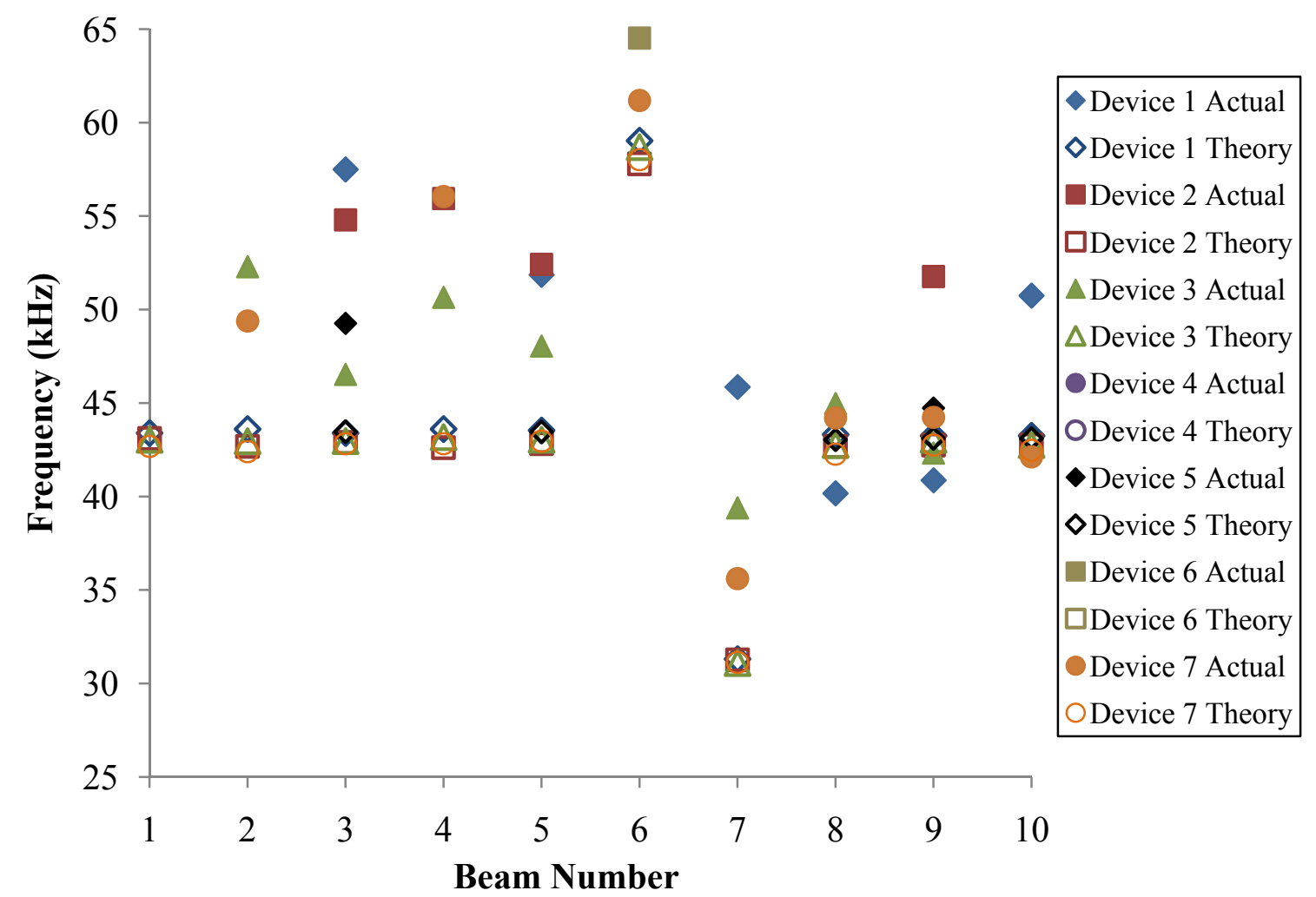

Figure 34 - Measured and (Theoretical/2) Resonant Frequencies of Cantilever Devices.

D. Static Deflection Tests

All microcantilever geometries on three devices were tested to verify their static sensitivity. Only three of the seven completed devices were tested because the static deflection test could not be performed on the four devices already soldered into the prototyping circuit boards (see Figure 23). Also, the static deflection test was harsh and caused failure in several of the tested microcantilevers. For each displacement increment in the SEM $(4 \mu \mathrm{m})$, a resistance was recorded. As an example, graphs of the measurements taken for two microcantilevers are shown in Figure 35 and Figure 36. All 
numerical results are specified in Appendix $\mathrm{C}$ and Figure 37 shows a graph of the static sensitivity data. The mean static sensitivities with error bars of one standard deviation and a constant cantilever beam length $(128 \mu \mathrm{m})$ are shown in Figure 38.

The average static sensitivity of the cantilever beams varied from $1.2 \Omega / \mu \mathrm{m}$ to 6.7 $\Omega / \mu \mathrm{m}$. The optimal microcantilever (beam 2) had a static sensitivity of $2.9 \pm 0.5 \Omega / \mu \mathrm{m}$. Sensitivity was not found for Beam 1 designs. Beam 2, 3, 4, and 5 samples had steadily decreasing sensitivities, in that order. Beam 6 had the highest mean static sensitivity and beam 7 also had relatively high sensitivity. Beam 8,9 , and 10 samples had steadily decreasing sensitivities, in that order, that were low in comparison to beam 2-5. Beam 8's result for Device 7 was classified as an outlier because it was twice as sensitive as any other Beam but had less optimum geometry. Beam 9's result for Device 3 was classified as an outlier because it is 5 times higher than the mean of the other two recorded data points. Finally, beam 10's result for Device 1 was classified as an outlier because it is more sensitive than the mean of beam 6 despite being the least sensitive geometric design and it is 10 times more sensitive than the other sensitivity data recorded for that microcantilever shape. 


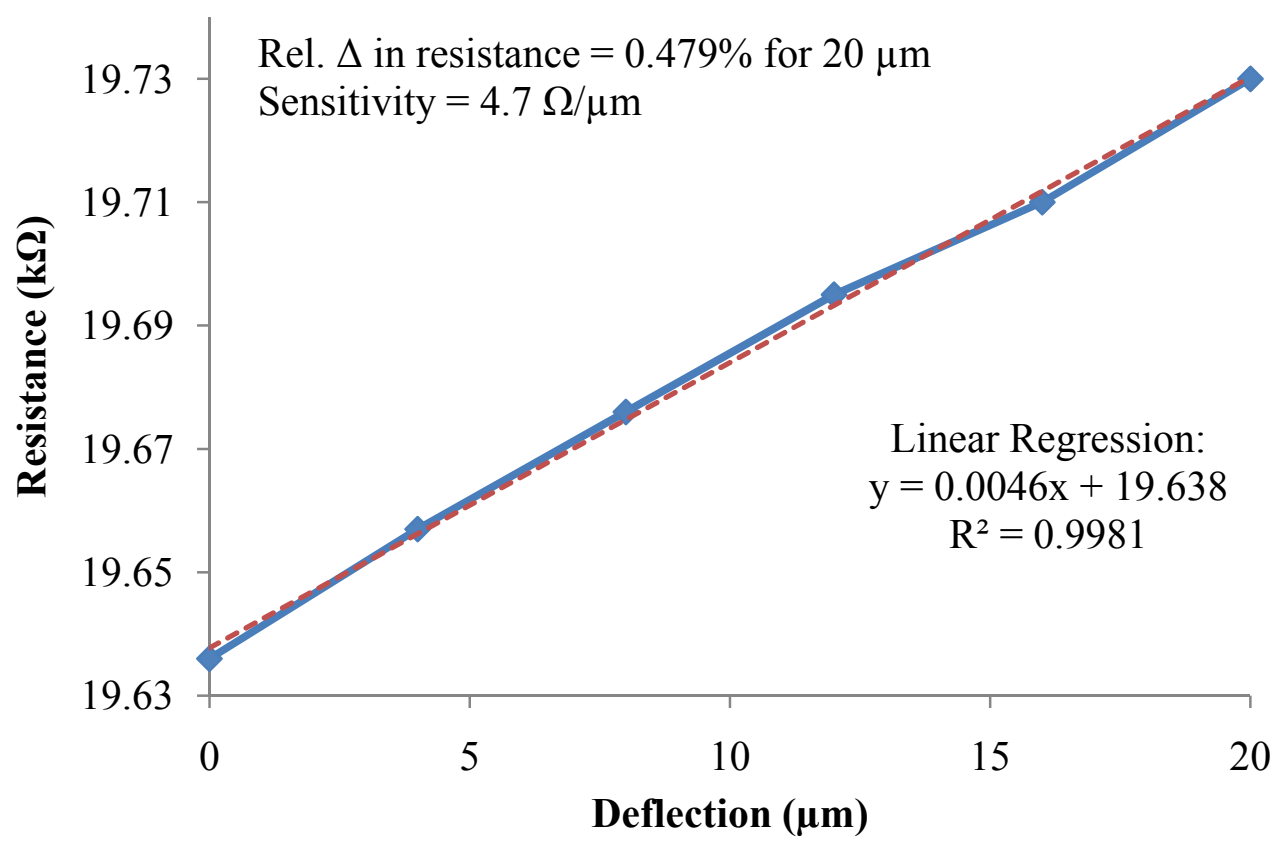

Figure 35 - Graph of Resistance Versus Deflection for Device 7, Beam 6.

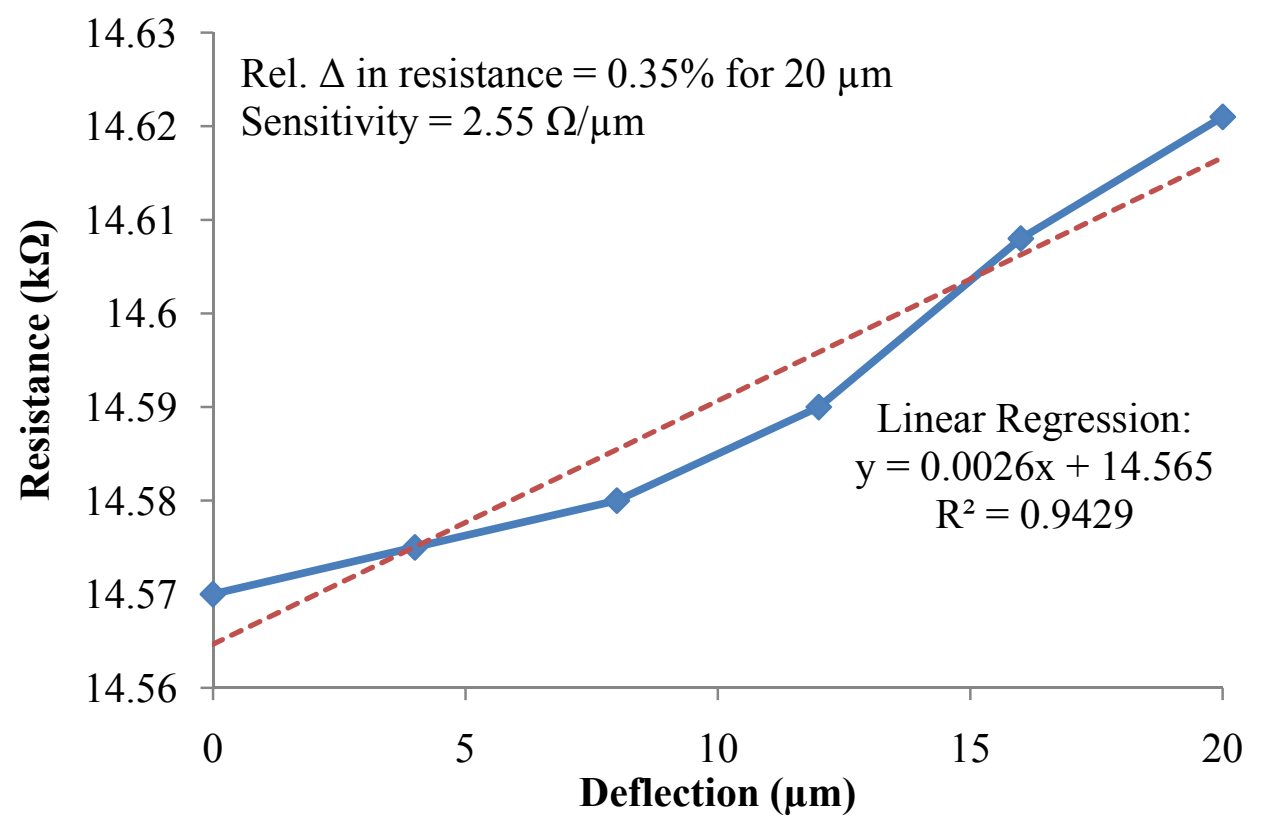

Figure 36 - Graph of Resistance Versus Deflection for Device 1, Beam 2. 


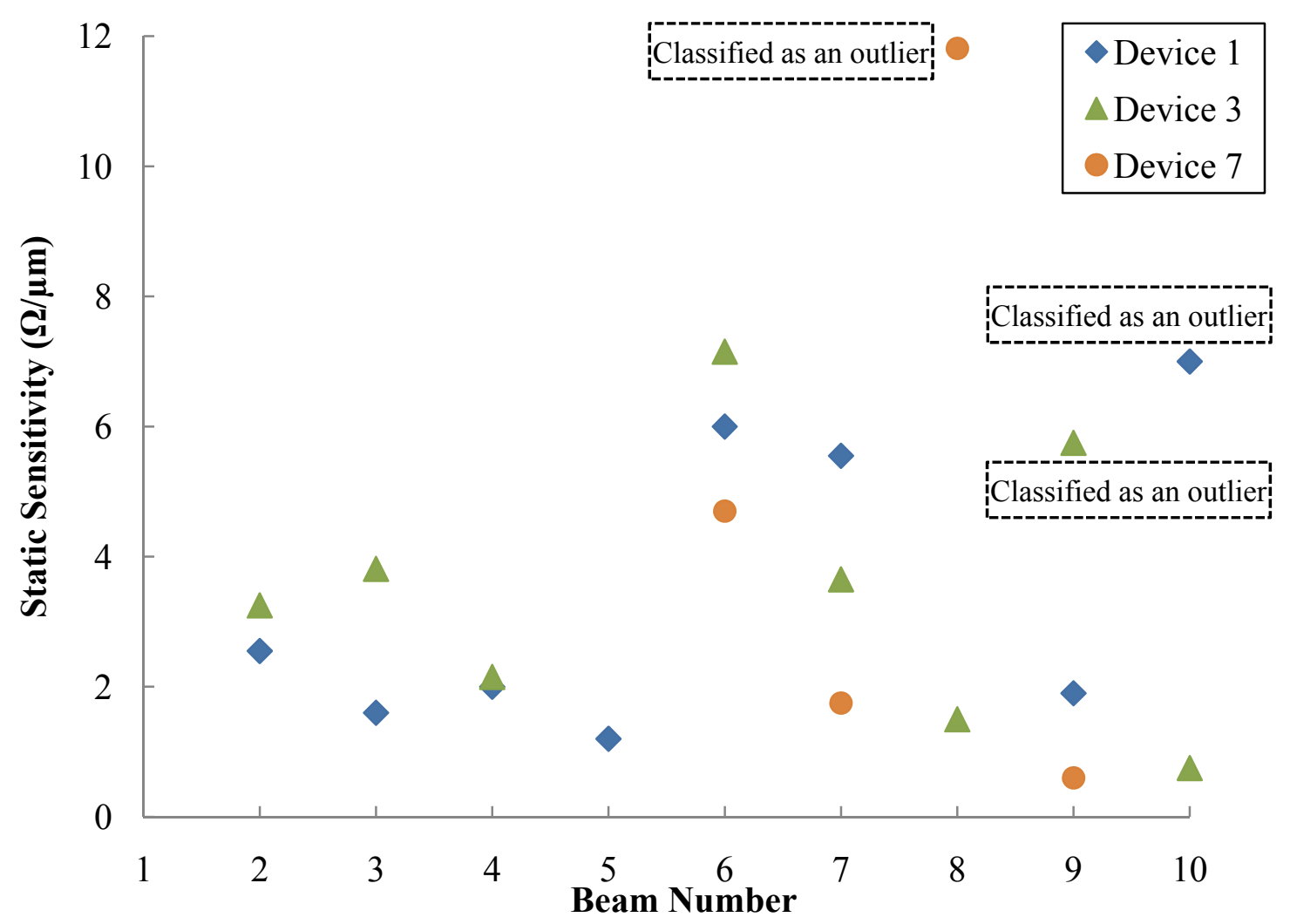

Figure 37 - Static Sensitivity of Cantilever Devices.

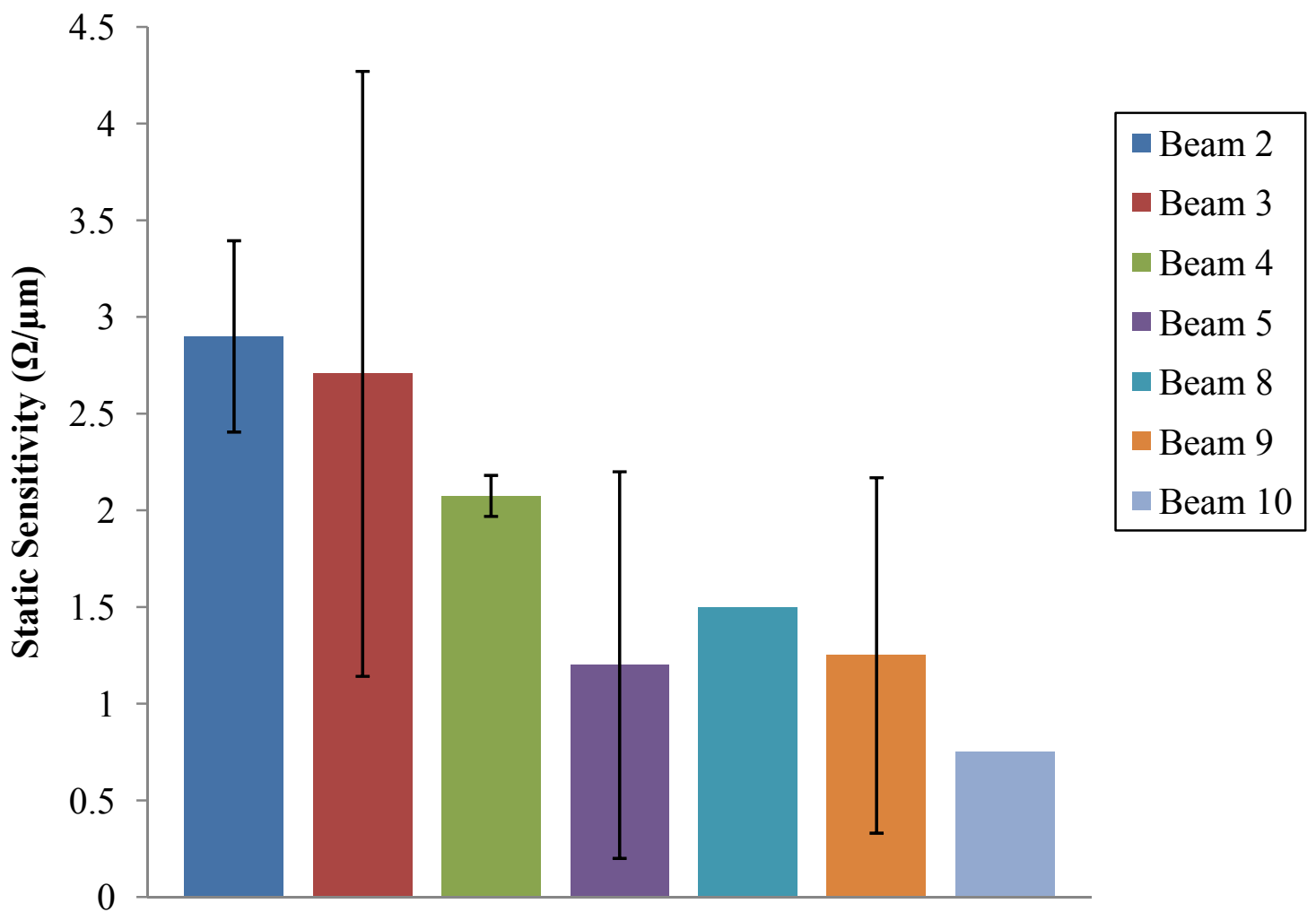

Figure 38 - Mean Static Sensitivity of Cantilever Devices, Beam Length Constant. 


\section{E. Dynamic Deflection Tests}

All microcantilevers with previously discovered resonant frequencies (in the probe station) on the seven devices were tested to verify their dynamic sensitivity. Only four of the seven completed devices had any microcantilevers that would resonate in the SEM chamber. All numerical results are specified in Appendix D and a graph of the dynamic sensitivity data are shown in Figure 39. The mean dynamic sensitivities with error bars of one standard deviation and a constant cantilever beam length $(128 \mu \mathrm{m})$ are shown in Figure 40. As stated previously, no beam 1 designs on the seven fabricated microcantilevers would resonate, so dynamic sensitivity data could not be gathered. The average dynamic sensitivity for the microcantilevers varied from $2.5 \Omega / \mu \mathrm{m}$ to $16.7 \Omega / \mu \mathrm{m}$. The microcantilever with the optimal piezoresistor geometry (beam 2) had an average sensitivity of $12.82 \Omega / \mu \mathrm{m}$, but only one data point was collected.

Similar to the static sensitivity results, beams $2,3,4$, and 5 had decreasing mean sensitivities, in that order, with beam 2 having the highest sensitivity in the group. No functioning beam 6 designs would resonate in the SEM chamber. Beam 7 devices had an average dynamic sensitivity of $16.73 \pm 3.95 \Omega / \mu \mathrm{m}$. This was the highest mean dynamic sensitivity out of all the beam designs. Beam 8 designs had an average dynamic sensitivity of $3.09 \pm 2.53 \Omega / \mu \mathrm{m}$ and beam 9 designs had an average dynamic sensitivity of $2.45 \pm 1.67 \Omega / \mu \mathrm{m}$. Beam 8 and 9 designs had relatively low mean sensitivities and no reliable data could be collected for beam 10 designs. Beam 10 on Device 1 was classified as an outlier because it was 8 times as sensitive as beam 9 , which had similar geometry. A comparison of static sensitivities and dynamic sensitivities for the tested beam designs is shown in Figure 41. Dynamic sensitivity is consistently higher than static sensitivity 
for each cantilever beam. Yang's (2006) best device sensitivity was $0.0231 \Omega / \mu \mathrm{m}$ versus the optimum device sensitivity reported here of $12.82 \Omega / \mu \mathrm{m}$, making the optimum piezoresistor design 550 times more sensitive than the symmetric piezoresistor reported previously.

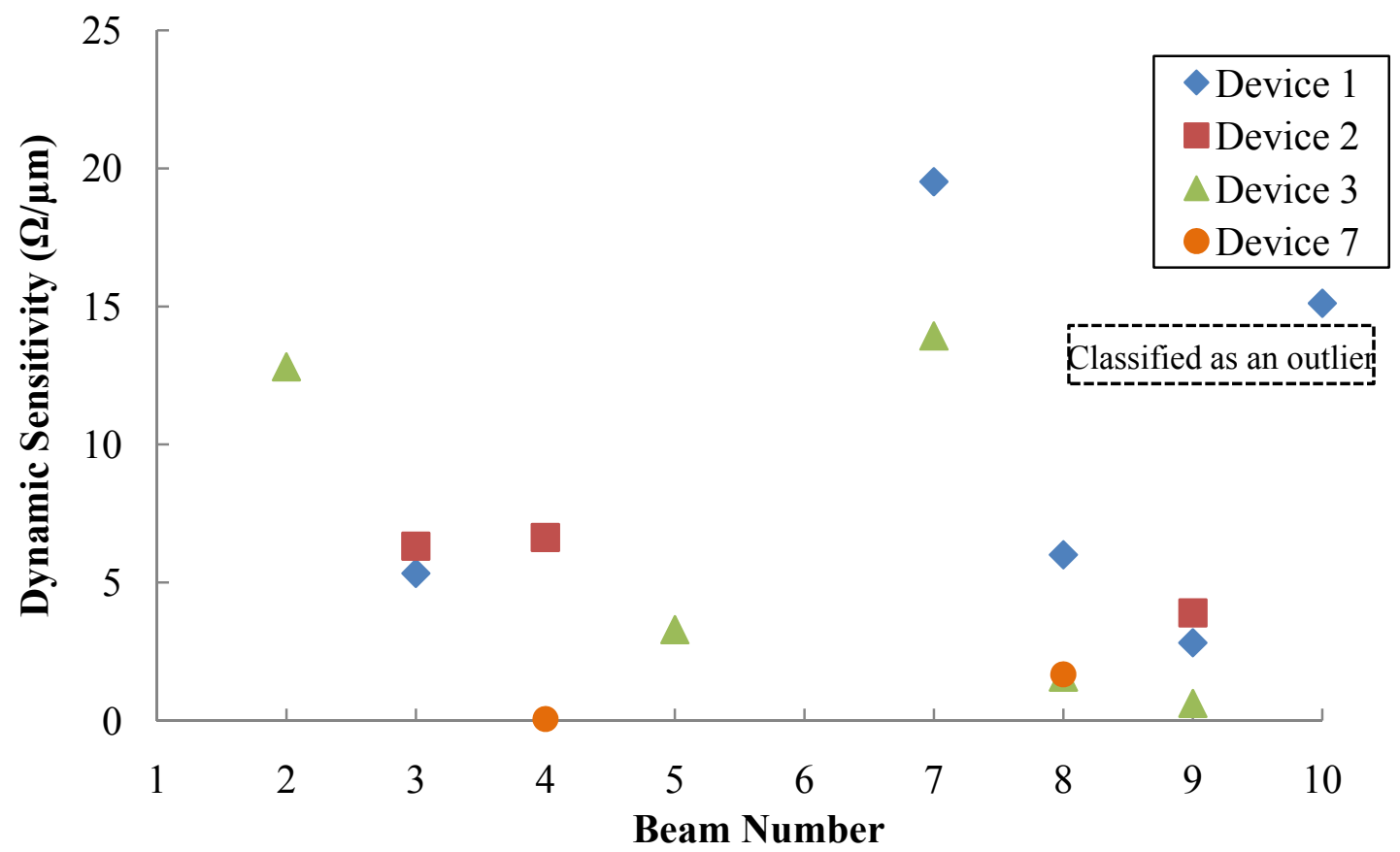

Figure 39 - Dynamic Sensitivity Of Cantilever Devices.

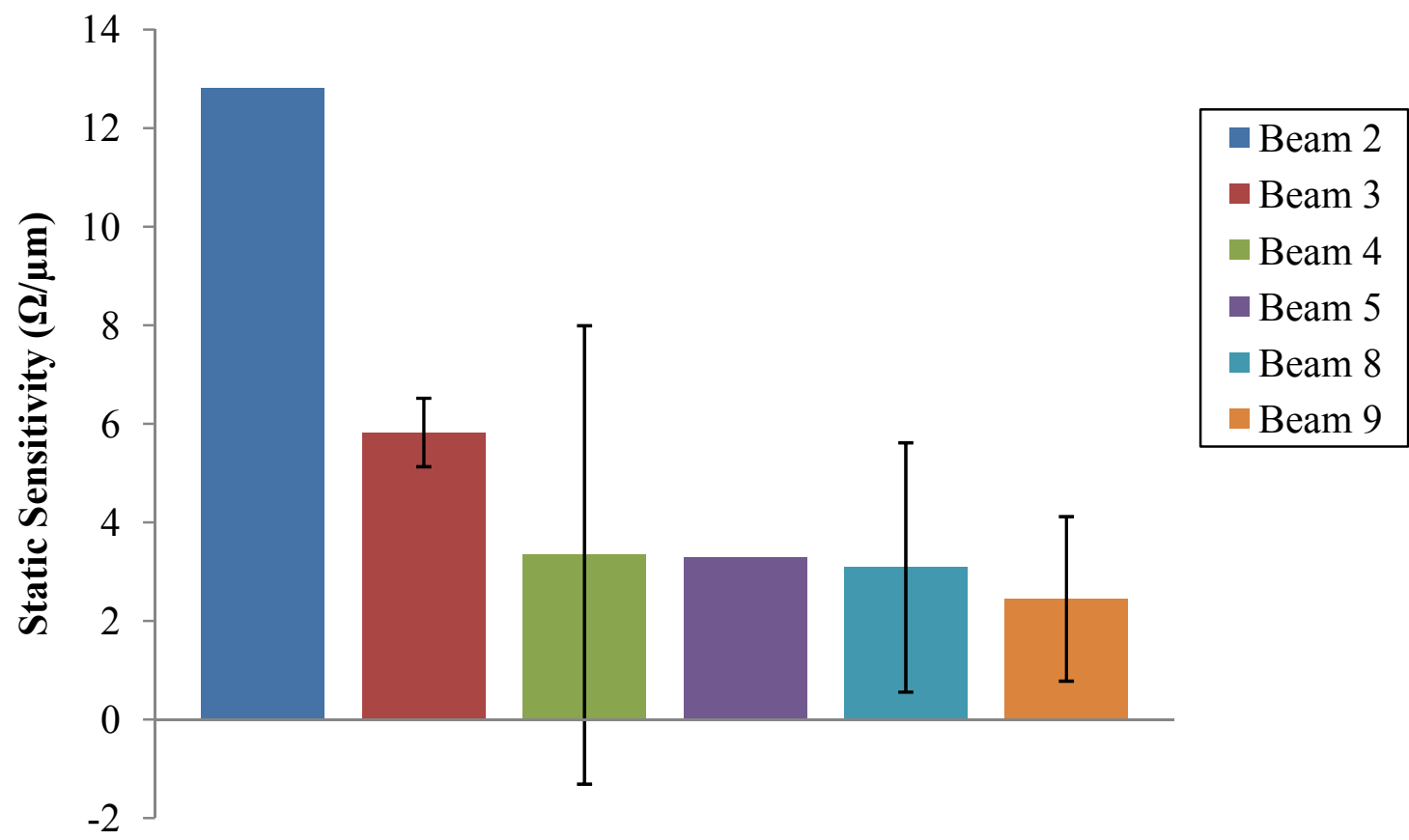

Figure 40 - Mean Dynamic Sensitivity of Cantilever Devices, Beam Length Constant. 


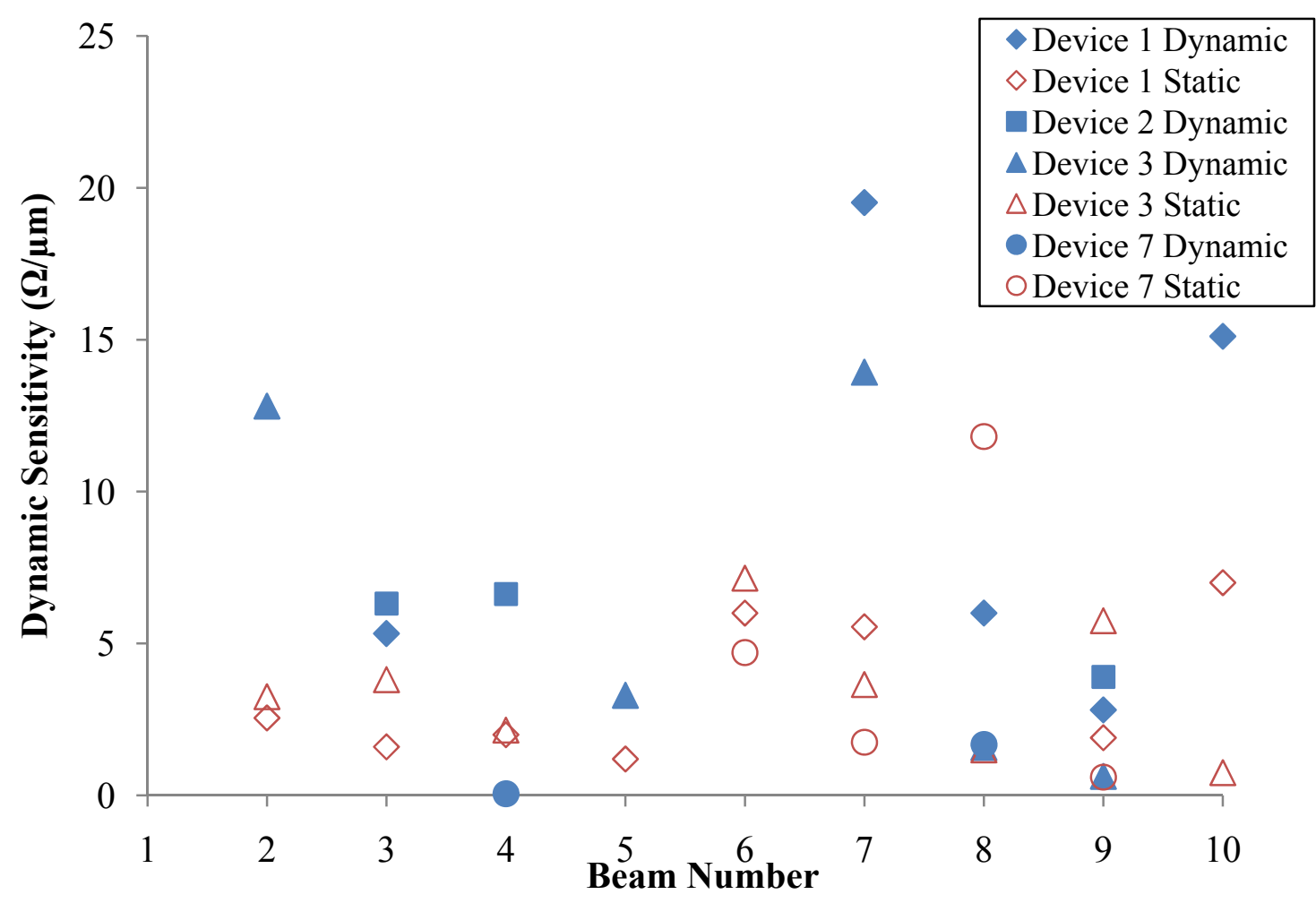

Figure 41 - Dynamic and Static Sensitivity Of Cantilever Devices. 


\section{DISCUSSION}

Laterally resonating microcantilevers were fabricated using the methods described in Section III. B. The resonator is based on a resonating cantilever whose base support acts as a piezoresistor. This MEMS resonator is driven electrostatically and deflection was detected using the piezoresistive sensing method. The piezoresistive base has been optimized to increase sensitivity over previous designs (Xu, 2006). The piezoresistive detector was characterized by investigating the relationship between the deflection of the cantilver and the change in resistance of the beam base using experimental analysis. The

new, asymmetric resonator was judged against the old symmetric resonator by comparing the static and dynamic sensitivities of the two designs. It was found that the optimized geometry results in a significant increase in device sensitivity. The implications of the results will be discussed below.

\section{A. Fabrication}

Initially, only Shipley 1827 photoresist was applied to the substrate before optical lithography processing. The result was a resist sidewall profile that had a positive slope and allowed metallization of the sidewall during sputtering. This made lift-off of large sacrificial gold areas very difficult because the acetone had to penetrate the metalized sidewalls. Lift-off took several days while soaking in acetone and was often incomplete. The solution was to apply LOR3A to the substrate prior to applying the Shipley 1827 photoresist. This addition created a negative slope in the resist sidewall and an overhang after photolithography development, preventing metallization of the resist sidewalls and promoting a discontinuous film deposition. The lift-off process was greatly improved and the time for complete lift-off was reduced to one hour in a recirculating acetone bath. 
The sputtering deposition rate for gold and chromium relies on many factors, including the vacuum base pressure, the argon working gas pressure, the DC sputtering power, and the RF sputtering power. Even after characterizing the deposition process, the deposition rate in the sputtering machine still varies widely from week-to-week. The gold deposited for the device pads and leads was thinner than desired on the outer edges of the SOI wafer because the sputtered metals were not deposited uniformly in a radial direction. Less gold on the bond pads makes it hard or impossible to wire bond from the gold pads using a wire bonder. It is recommended that the deposition rates for the sputtering machine be checked a few days before sputtering on the SOI wafer to improve device yield and ease of wire bonding.

Initially, during e-beam lithography all ten microcantilevers on each device were written simultaneously so that only one alignment had to be performed to save time. Subsequently, the SEM and NPGS system were set on very low magnifications so that they could image the entire substrate. The result was incomplete exposure of every cantilever, a lack of needed resolution in the structures, and poor alignment at the outer edges of the e-beam exposure (see Figure 42 for an example). The solution was to align and expose each microcantilever individually, which added time and complicated the ebeam lithography procedure. Also, alignment for the microcantilever was performed on the nearest trace corner which exposed the corner resist, forming openings in the resist after development that were filled with iron during the e-beam evaporation. After DRIE, this resulted in silicon artifacts at the alignment points (see Figure 43 for an example). However, these artifacts did not affect device performance and individually exposing each microcantilever was a success. 


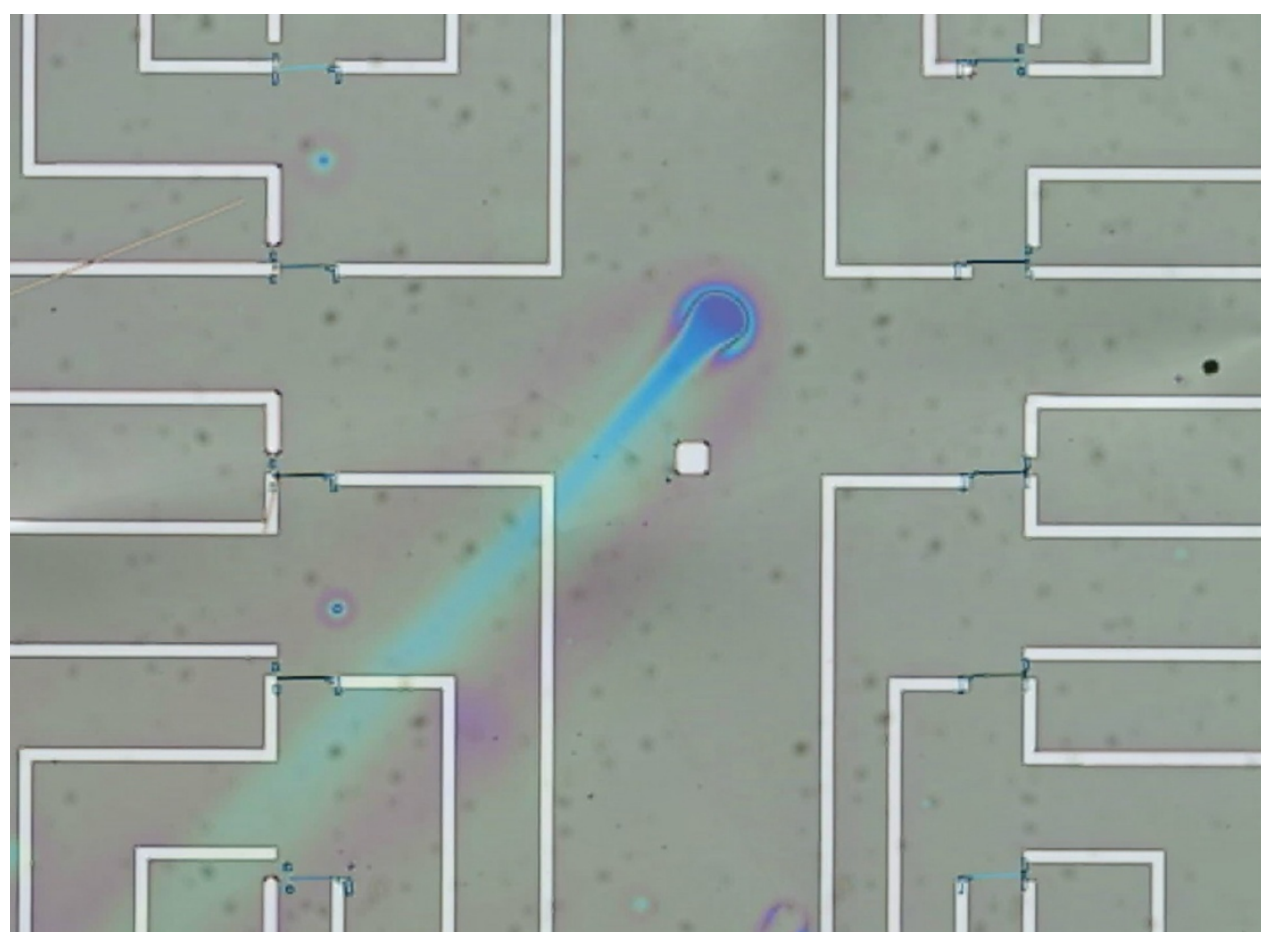

Figure 42 - E-Beam Exposure of Ten Microcantilevers Simultaneously After Development.

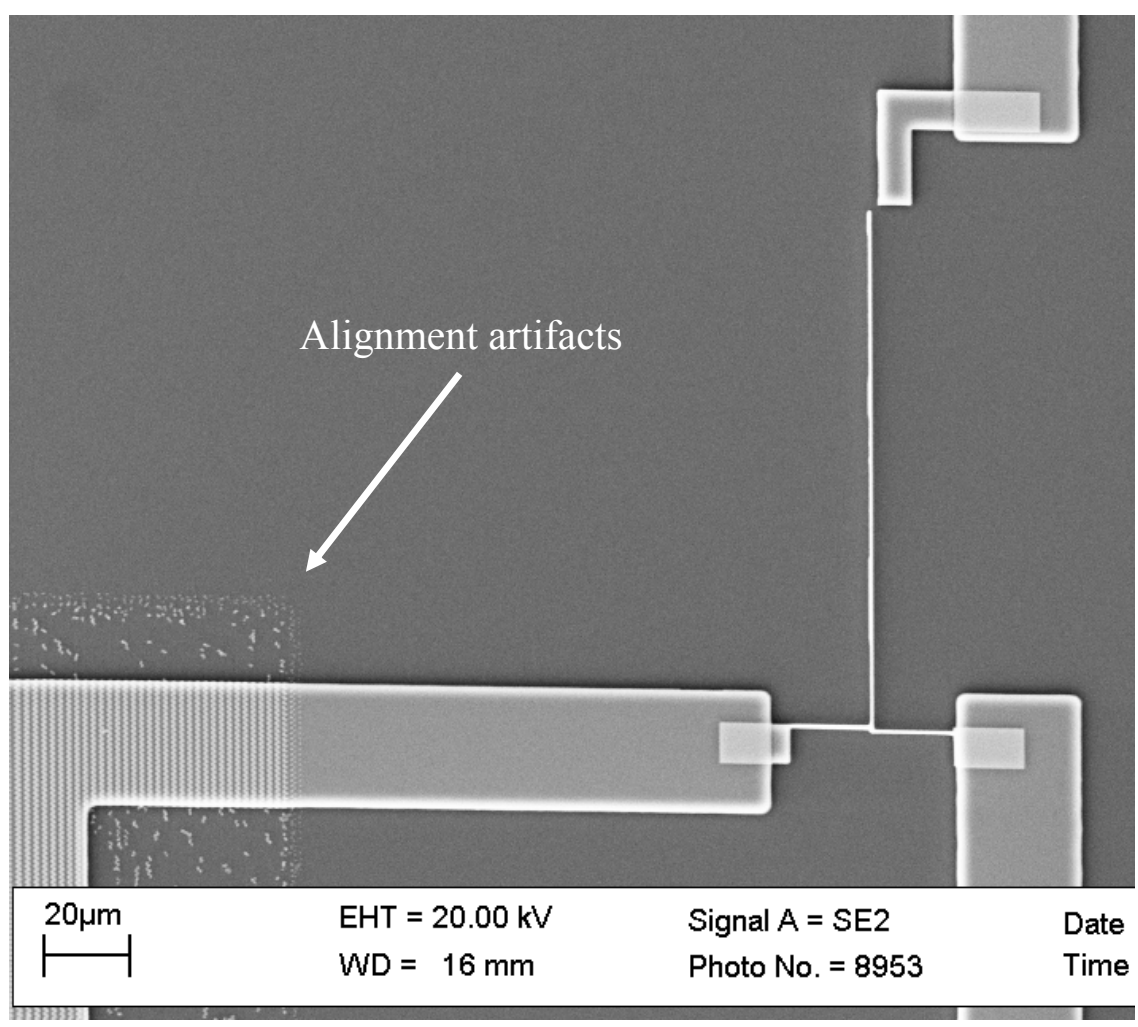

Figure 43 - Alignment Artifacts From E-Beam Writing. 
Initially, the DRIE etch was performed for 12 minutes in one lump sum that was only marginally successful. If overetched, the iron mask would begin to peel off and etching of the microcantilever beam material would commence (example shown in Figure 44). Alternatively, the etch could be performed in increments, while still maintaining straight sidewalls, so that the etch progress could be checked incrementally. This change in procedure increased device yield to almost $100 \%$.

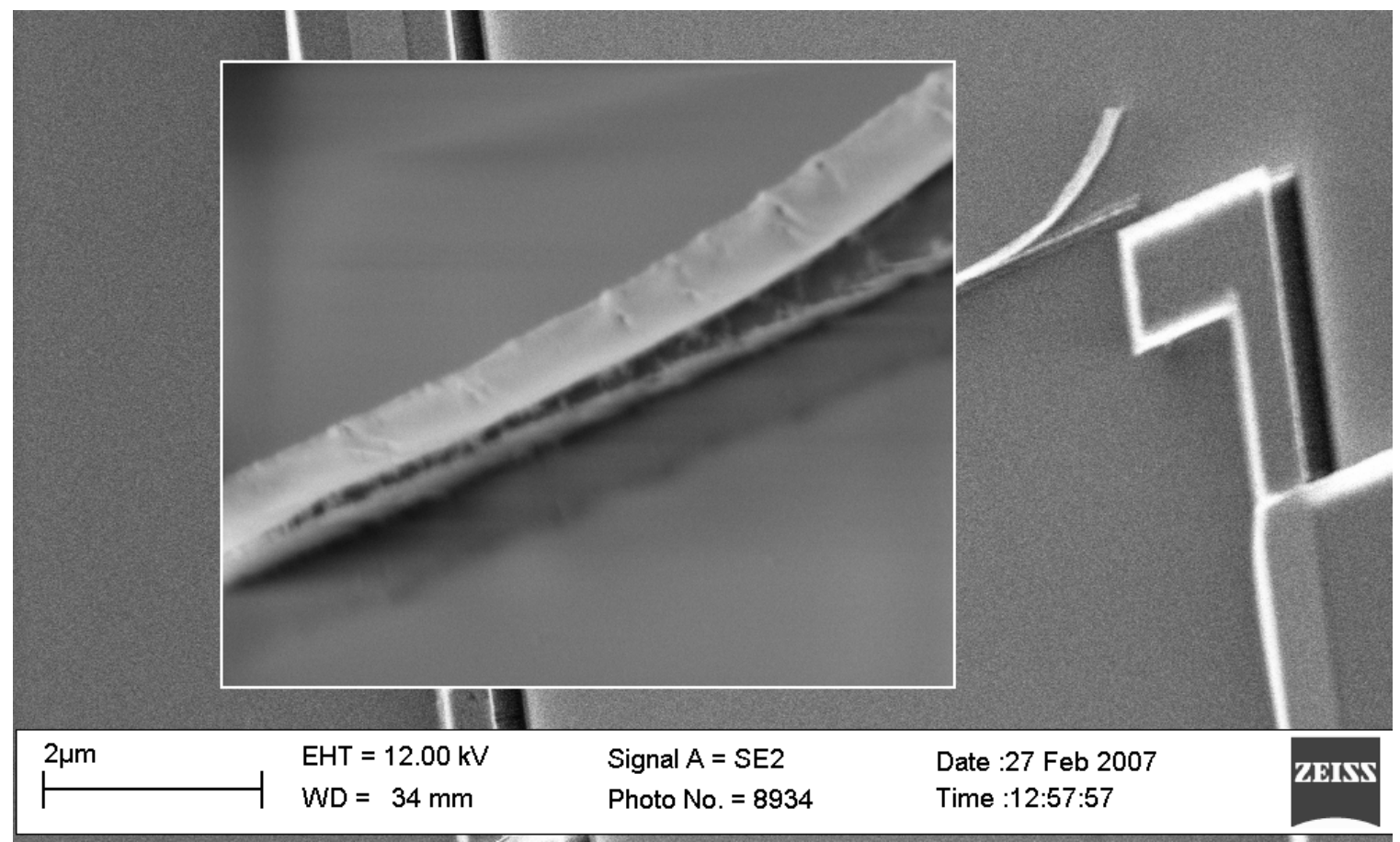

Figure 44 - Overetching Microcantilever in DRIE; Iron Masking Layer Peeling Off.

Drying MEMS structures in close proximity to other structures or a substrate is complicated by the tendency for the surface tension of the liquid to pull one structure in contact with another. The structures tend to stay in contact after being dried. An example of a microcantilever that was removed from a BOE wet etchant, rinsed in DI water, and then placed in IPA before being critical point dried is shown in Figure 45. Placement of the "stuck" microcantilever structures in IPA and briefly dunking them in an ultrasonic 
bath is not a viable solution, as evidenced by Figure 46. The microcantilever was completely destroyed in the ultrasonic bath.

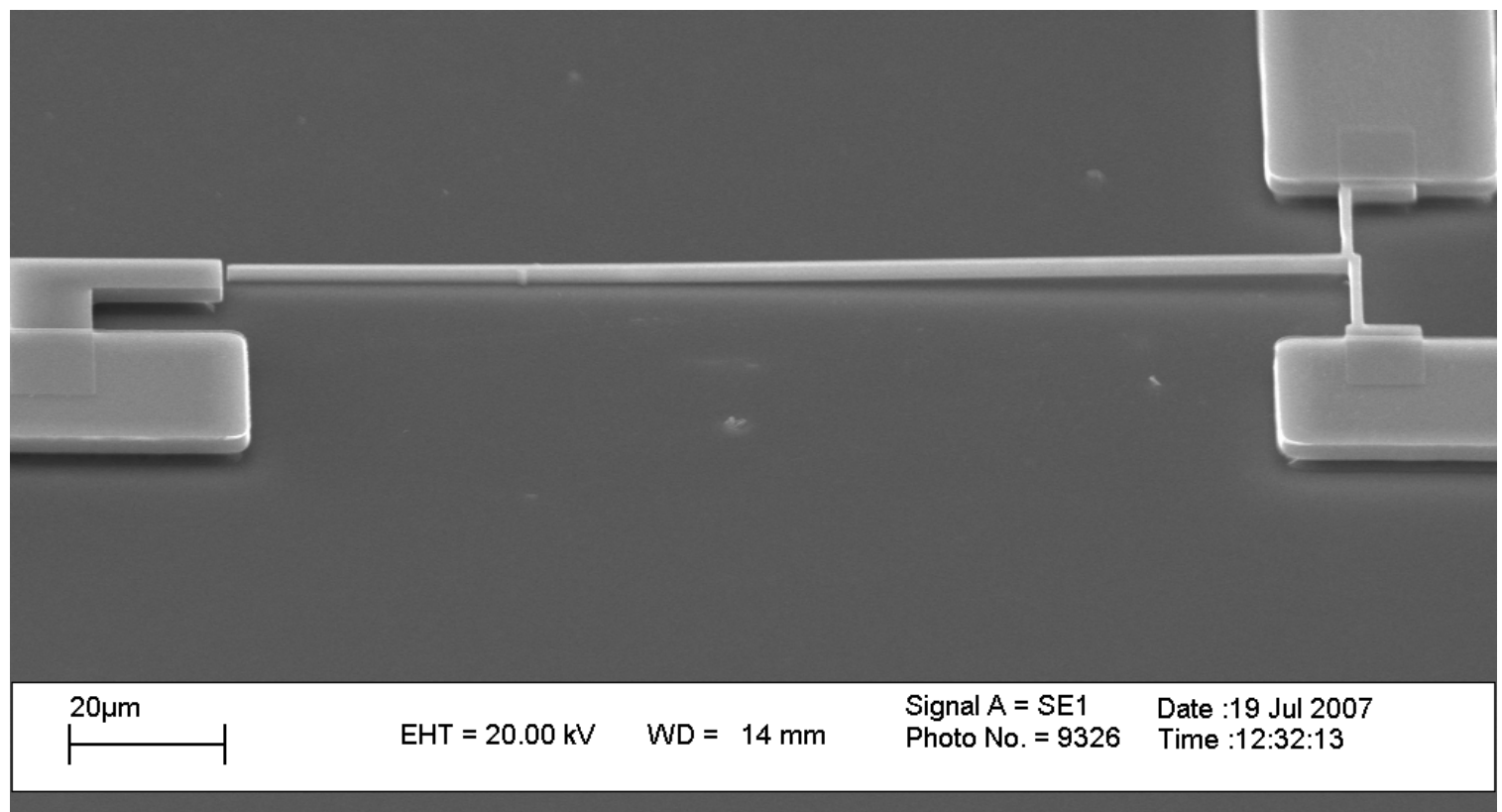

Figure 45 - SEM Micrograph of a Microcantilever That Was Removed From the Liquid BOE and Rinsed in DI Water and IPA Before Critical Point Drying.

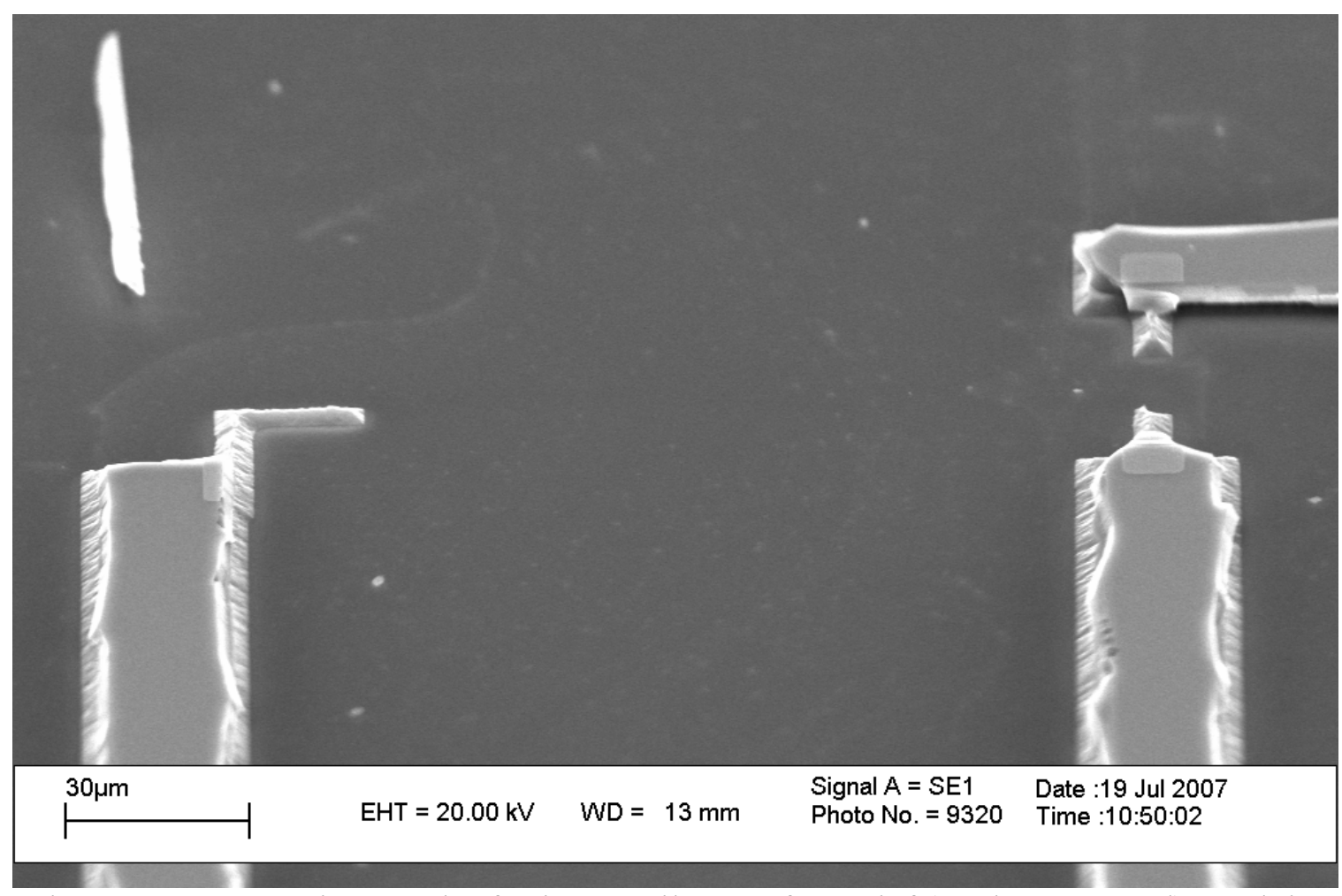

Figure 46 - SEM Micrograph of Microcantilever After Brief (Under 1 Second) Dunk in Ultrasonic Bath. 
The solution to this stiction problem was presented in the Critical Point Drying Process section of Materials and Methods. Removing the BOE and DI water from the device die before critical point drying was accomplished through a liquid displacement and aspiration process. This improved drying method avoided the stiction problem and significantly increased device yield to almost $100 \%$ after its implementation.

\section{B. Determination of Static Piezoresistor Resistance}

Resistance across the zero-deflection piezoresistor for each geometric design was measured to determine whether the DRIE process had critically damaged the electrical conducting capability of the boron-doped silicon bridge. Xu (2006) observed that there was a critical thickness below which electrical conductivity was severely restricted. She noted that an average piezoresistor width of $1.03 \mu \mathrm{m}$ had resistances near $11 \mathrm{k} \Omega$, while an average thickness of $0.54 \mu \mathrm{m}$ or $0.16 \mu \mathrm{m}$ had resistances on the order of tens of Gigaohms. Her T-9 devices had an average piezoresistor width of $1.1 \mu \mathrm{m}$.

The new asymmetric microcantilevers had an average piezoresistor width of 1.26 $\mu \mathrm{m} \pm 0.08 \mu \mathrm{m}$ and the same average thickness as the piezoresistors reported by $\mathrm{Xu}$, et al., $2.0 \mu \mathrm{m} \pm 0.5 \mu \mathrm{m}$. As shown in Table III, the asymmetric microcantilevers had resistances from $12 \mathrm{k} \Omega$ to $17 \mathrm{k} \Omega$. A $14 \mathrm{k} \Omega$ resistor was paired with the piezoresistor in the voltage dividing circuitry, shown in Figure 17 and Figure 25, because it was a good average value of the resistance measurements and could be used in the voltage divider for all the microcantilevers. These resistance values are in line with Yang's measurements; the higher resistances are associated with thinner piezoresistors and the variation in resistances can be attributed to fabrication uncertainty. Several fabrication steps affect the piezoresistance of the microcantilever base. The DRIE process has been shown to 
damage the electrical conductivity, to some depth, of boron-doped silicon (Xu, 2006), so variations in the DRIE etch time altered the extent of conductivity damage. Also, the variance in smoothness of the iron masking layer affected the maximum conductivity capability of the piezoresistor, ie. notches in the piezoresistor dictated the effective width of the piezoresistor. Finally, the variance in piezoresistor thickness caused limits in the total current carrying capacity of the doped silicon bridge.

\section{Microcantilever Metrology}

Every fabricated microcantilever's geometry was carefully measured to compare the desired geometry to the actual device geometry. The averages for each beam design were given in Figure 26 through Figure 29 and the percent errors in the measured values are given in Figure 30 through Figure 33. The average "leg length" parameter for actual devices tended to be $0.5-2 \mu \mathrm{m}$ shorter than desired. This is attributed to calibration errors in the Nanometer Pattern Generation System (NPGS), variability in the e-beam development process, variability in the iron deposition masking layer, and variability in the DRIE etching process. In the NPGS system, the magnification value used in the SEM did not properly correspond to the magnification value input in the NPGS system, causing the written images to be slightly too big or too small. The e-beam development of small features hinges very precisely on development time, freshness of the three photoresists on the substrate, freshness of the developing medium (MIBK), and proper exposure of the pattern. Resist overhangs formed during development limit the deposition area of the iron masking layer, causing some features to be too thick or too thin. Finally, the uncertainty of the DRIE undercutting of the iron masking layer caused the microcantilever beam and piezoresistor to be thicker or thinner than desired. 
The average "offset" parameter for actual devices varied from $0-0.25 \mu \mathrm{m}$ from the dimensions desired. Again, these errors can be attributed to fabrication errors discussed in relation to the "leg length" parameter. The average "beam length" parameter for devices was consistently 6-7 $\mu \mathrm{m}$ shorter than desired. Yet again, these errors can be attributed to fabrication errors discussed previously, but the consistent error with little standard deviation suggests a consistent problem with the e-beam lithography process. The image produced by the NPGS system was most likely consistently undersized, suggesting a recalibration of the system is required. In addition, the NPGS "center-to-center" distance and "line width" distance affect how closely spaced the electron beams are that expose the resist. These values may need to be adjusted by recalibrating the e-beam lithography process. Also, the pattern development in MIBK may have been too short such that the exposed pattern was not completely opened up.

Finally, the piezoresistor "beam width" parameter had a large amount of variability and averaged 0.1-0.3 $\mu \mathrm{m}$ thicker than intended. This phenomenon stems from an overmagnification of the desired pattern in the NPGS system, e-beam currents and doses that were too high for the intended resist target, and overdevelopment of the exposed pattern in MIBK. Also, scattering of the e-beam in the resist causes the polymer chains to break molecular bonds on either side of the electron beam path, making the developed path susceptible to solvent wider than intended. These e-beam characteristics make the NPGS exposed pattern wider than intended during development and the subsequent etching steps.

Errors in the microcantilever dimensions cause the piezoresistance, sensitivity, and resonant frequency to be different than desired. Shorter leg lengths after fabrication make 
the overall piezoresistor shorter, resulting in lower resistances. Similarly, the thicker beam widths provide more cross-sectional area for conductivity in the piezoresistor and therefore lower the resistance of the piezoresistor. The thicker cantilever beam width results in additional mass, lowering the resonant frequency of cantilever. Conversely, the consistently shortened cantilever beams have less mass than intended and resonate at higher frequencies than beams with correct lengths.

D. Resonator Actuation Experiments

In general, the newly-designed asymmetric cantilevers with beam lengths of 128 $\mu \mathrm{m}$ resonated between $43 \mathrm{kHz}$ and $54 \mathrm{kHz}$, depending on the piezoresistor base geometry. This is in agreement with Yang's (2006) T-shaped microcantilevers (base beams $9 \mu \mathrm{m}$ in length), whose resonant frequencies varied between $42 \mathrm{kHz}$ and $50 \mathrm{kHz}$. The beam 6 designs tended to yield higher resonant frequencies, $62,856 \mathrm{~Hz} \pm 2,356 \mathrm{~Hz}$, due to the shorter cantilevers (110 $\mu \mathrm{m}$ long). In comparison, beam 7 designs had lower resonant frequencies, $40,290 \mathrm{~Hz} \pm 5,182 \mathrm{~Hz}$, because the cantilever lengths were longer $(150 \mu \mathrm{m})$. Microcantilever resonant frequencies are inversely related to the length of the cantilever and the mass of the cantilever beam. The resonant frequency of a cantilever is given by

$$
w_{o}=\sqrt{\frac{k}{m}}
$$

where $m$ is the mass of the cantilever and $k$ is the cantilever spring constant, defined as

$$
k=\frac{E w t^{3}}{4 L^{3}}
$$

in which E is Young's modulus of the cantilever material, $w$ is the cantilever width, $t$ is the cantilever thickness, and $\mathrm{L}$ is the beam length. The theoretical resonant frequencies calculated and shown in Figure 34 agree fairly well with the measured resonant 
frequencies. Discrepancies could be caused by differences in the material properties of the crystalline silicon than those used in the equations. Also, errors differences in the cantilever thickness would cause errors in the value calculated for the cantilever resonant frequency. Finally, the base of the cantilever is not completely fixed because of the suspended piezoresistor and therefore does not behave exactly as predicted by Equations 8 and 9.

The beam 1 cantilever design served as a control for Xu, et al.'s (2006) T-shaped design; however, it did not resonate when the drive frequency was swept from 0 to 80 $\mathrm{kHz}$ in $1 \mathrm{~Hz}$ increments and the drive electrode was actuated with an $\mathrm{AC}$ voltage of 10 Volts peak-to-peak, which is the maximum output of the function generator ( Agilent 33220A). Possible sources for this phenomenon include thick piezoresistor geometry (average of $1.38 \mu \mathrm{m}$ when $1.1 \mu \mathrm{m}$ was the intended thickness), short piezoresistor legs (average of $3.93 \mu \mathrm{m}$ when $4.5 \mu \mathrm{m}$ was the intended length), piezoresistor damage from DRIE, or a resonating frequency higher than $80 \mathrm{kHz}$. These dimensional errors are relatively significant; since the beam length and piezoresistor legs are short but the piezoresistor and beam are too wide, it is most likely that the overall e-beam pattern was too small and overexposed, resulting in short line lengths that became too thick when developed in MIBK. Since the static resistance tests showed reasonable values, the piezoresistors are conductive. The drive signal (10 Volts peak-to-peak) may not provide enough electrostatic potential to bring the thick cantilever beam into resonance.

\section{E. Static Deflection Tests}

Beam 3 had very similar geometry to beam 2 and thus had a static sensitivity very close, but lower, $2.7 \pm 1.6 \Omega / \mu \mathrm{m}$, than that of beam 2 . Beam 4 deviated even more from 
the optimum geometry and therefore the static sensitivity was even lower $(2.1 \pm 0.1$ $\Omega / \mu \mathrm{m})$. The beam 5 design used a thicker piezoresistor base $(1.3 \mu \mathrm{m})$ to help study DRIE conductivity damage. This larger base resulted in a lower stress in the piezoresistor, making it less sensitive. This was corroborated when the tested device yielded a static sensitivity of only $1.2 \Omega / \mu \mathrm{m}$.

Beam 6 and beam 7 used the optimal piezoresistor geometry, but had shorter and longer cantilever beams, respectively. A $20 \mu \mathrm{m}$ displacement at the tip of beam 6 is not equivalent to a $20 \mu \mathrm{m}$ displacement at the tip of beam 2 ; the tip of beam 2 rotates $9.0^{\circ}$ while the tip of beam 6 rotates $10.5^{\circ}$, yielding a larger piezoresistor stress than beam 2 . Thus, beam 6 produced the highest static sensitivity recorded for the microcantilevers $(6.0 \pm 1.2 \Omega / \mu \mathrm{m})$. Similarly, a $20 \mu \mathrm{m}$ displacement at the tip of beam 7 is slightly less than a $20 \mu \mathrm{m}$ displacement at the tip of beam 2 ; the tip of beam 2 rotates $9.0^{\circ}$ while the tip of beam 7 rotates $7.7^{\circ}$. Beam 7 had a static sensitivity of $3.7 \pm 1.9 \Omega / \mu \mathrm{m}$, which was higher than the optimum design (beam 2), but also had a larger standard deviation in sensitivity. This larger standard deviation could be caused by noise in the sensing circuitry, non-optimal orientation of the microcantilevers in the $<110>$ crystal plane, or inconsistency in fabrication techniques.

Beams 8,9 , and10 possessed a shorter piezoresistor length (PL) while varying the leg separation and leg length. The static sensitivity of beams 8,9 , and 10 were $1.5 \Omega / \mu \mathrm{m}$, $1.25 \pm 0.9 \Omega / \mu \mathrm{m}$, and $0.8 \Omega / \mu \mathrm{m}$, respectively. Only one beam was successfully tested for each of beam 8 and 10. Some resistance versus deflection measurements were not as linear as others (Figure 35 versus Figure 36). This is most likely due to errors in the measurement setup for the static deflection tests. Each deflection increment was not 
accurately $4 \mu \mathrm{m}$ because the nanomanipulators could not reliably move the tungsten needles according to the overlaid grid. The tip of the cantilever was also visually lined up with the overlaid grip, which lacks precision and introduces error. Finally, the electron beam of the SEM causes charging in the structures it images, which makes the resistance measurements less accurate.

In summary, as the leg separation (LS) increases and the leg length (LL) decreases, but the piezoresistor length (PL) remains constant, the static sensitivity of the piezoresistor reduces. Eliminating beam length as a variable (ignoring beam $6 \& 7$ ), the static sensitivity data shows that the most sensitive piezoresistor has an optimum beam geometry of $\mathrm{LS}=1 \mu \mathrm{m}, \mathrm{LL}=20 \mu \mathrm{m}$, and $\mathrm{PL}=41 \mu \mathrm{m}$. This has been corroborated in Finite Element Analysis (FEA) of the asymmetric microcantilever (Bradshaw, et al. 2007).

\section{F. Dynamic Deflection Tests}

Beam 3 had a lower average dynamic sensitivity $(5.82 \pm 0.70 \Omega / \mu \mathrm{m})$ than the optimum design for reasons discussed in the static sensitivity section; similarly, beam 4 had a lower average dynamic sensitivity $(3.34 \pm 4.65 \Omega / \mu \mathrm{m})$ than beam 3 when compared to beam 2. No data for dynamic sensitivity of beam 6 designs was collected because none of these beams resonated in the SEM chamber. Only 2 devices resonated in the probe station and none would resonate in the SEM after significant exposure to room air. A considerable amount of particulate debris was observed on all the device surfaces by the time they were ready for dynamic sensitivity testing, with many particles ending up on beam 6 designs. Beam 7 devices had an average dynamic sensitivity significantly higher than the sensitivity of beam 2 . However, beam 7 devices used the same piezoresistor 
geometry but had cantilever beams which were $22 \mu \mathrm{m}$ longer than the cantilevers for beam 2 . This resulted in lower resonate frequencies $(\sim 40 \mathrm{kHz})$ but larger resonate displacements ( $\sim 40 \mu \mathrm{m}$ for beam 7 but only $\sim 17 \mu \mathrm{m}$ for beam 2 ). If beam 2 microcantilevers had resonant displacements similar to the distance displaced by beam 7 microcantilevers, they would also have higher dynamic sensitivities. This explains why the beam 7 microcantilevers have higher dynamic sensitivities than their beam 2 counterparts.

The dynamic sensitivities for beams $8(3.09 \pm 2.53 \Omega / \mu \mathrm{m})$ and $9(2.45 \pm 1.67$ $\Omega / \mu \mathrm{m}$ ) followed a down-sloping trend similar to the static sensitivity trend described previously. No data was successfully collected for beam 10 microcantilevers because only one would resonate in the SEM chamber and its dynamic sensitivity data appeared to be an outlier. Since beam 1 designs were not successfully brought into resonance in the probe station, their dynamic sensitivity could not be tested. The dynamic sensitivities of the cantilever beams were consistently higher than their static sensitivities. This is most likely a result of the cyclic loading introduced at the piezoresistor base during dynamic testing. Joule heating and mechanical damping of the oscillating structures raises the temperature of the piezoresistor, thereby increasing the resistance of the piezoresistor. When the structure is dynamically actuated but is not in resonance, the mechanical damping does not create as much heat as when it is in resonance and the tip deflection is significantly higher $(\sim 1 \mu \mathrm{m}$ when not in resonance versus $\sim 17 \mu \mathrm{m}$ when in resonance). Therefore, the piezoresistor becomes hotter when in resonance and the dynamic sensing mode is more sensitive than the static sensing mode. 
A lack of working devices was the result of fabrication damage to the piezoresistor from DRIE and BOE etching, stiction of the devices to the substrate, and debris on the microcantilever after significant exposure to room air during epoxy, wire bonding, and soldering actions. Device yield could be significantly increased if these actions were performed in a cleanroom environment. Again eliminating beam length as a variable (ignoring beam $6 \& 7$ ), the dynamic sensitivity data shows that the most sensitive piezoresistor has an optimum beam geometry of $\mathrm{LS}=1 \mu \mathrm{m}, \mathrm{LL}=20 \mu \mathrm{m}$, and $\mathrm{PL}=41$ $\mu \mathrm{m}$. This has been corroborated in finite element analysis (FEA) of the asymmetric microcantilever (Bradshaw, et al. 2007).

These asymmetric microcantilevers are significantly more sensitive than the symmetric versions, and will therefore be more accurate in performing gas composition analysis using the viscous damping principal. While the change in natural frequency caused by viscous damping will be the same regardless of piezoresistor geometry, the larger change in resistance as a result of the redesigned sensing element will be much easier for measurement electronics to detect. This increase in sensitivity will make it possible for less power to be used in the measurement circuit and subsequently allow the measurement to be performed by on-chip CMOS electronics rather than lab-grade equipment. Using asymmetric microcantilevers for applications in which symmetric cantilevers have traditionally been used offers the potential to radically improve existing sensor sensitivity. 


\section{CONCLUSIONS AND RECOMMENDATIONS}

Lateral vibrating silicon resonators have been successfully fabricated and the fabrication technique has been documented. A new method for drying the microcantilevers to avoid stiction was developed. Piezoresistive sensing has been successfully used to detect resonance, avoiding complicated and bulky optical detection methods. Experimental studies have been performed to characterize 10 different piezoresistor geometries and compare the results to computational models (Bradshaw, et al. 2007). The optimum asymmetric piezoresistor geometry was found to be more sensitive than the symmetric piezoresistor geometry, as reported previously by Dr. Yang $\mathrm{Xu}(2006)$.

The microcantilevers developed in this study can be used for detecting the molar mass of a gas environment based purely on the viscous damping effect using a similar protocol as defined by $\mathrm{Xu}$, et al. (2006). Further studies using these asymmetric microcantilever arrays should investigate resonator response to gaseous environments and pursue the use of these devices for obtaining a 'signature' for a gas during composition analysis. The fabrication process should be further refined to achieve optimal device thicknesses of $1.1 \mu \mathrm{m}$ while maintaining other geometric parameters. 


\section{APPENDIX A - LABVIEW ALGORITHM}

The following are screenshots of the entire LabVIEW virtual instrument Sweep_Freq2.vi used to control instruments in the measurement circuit (Figure 17) and collect data.

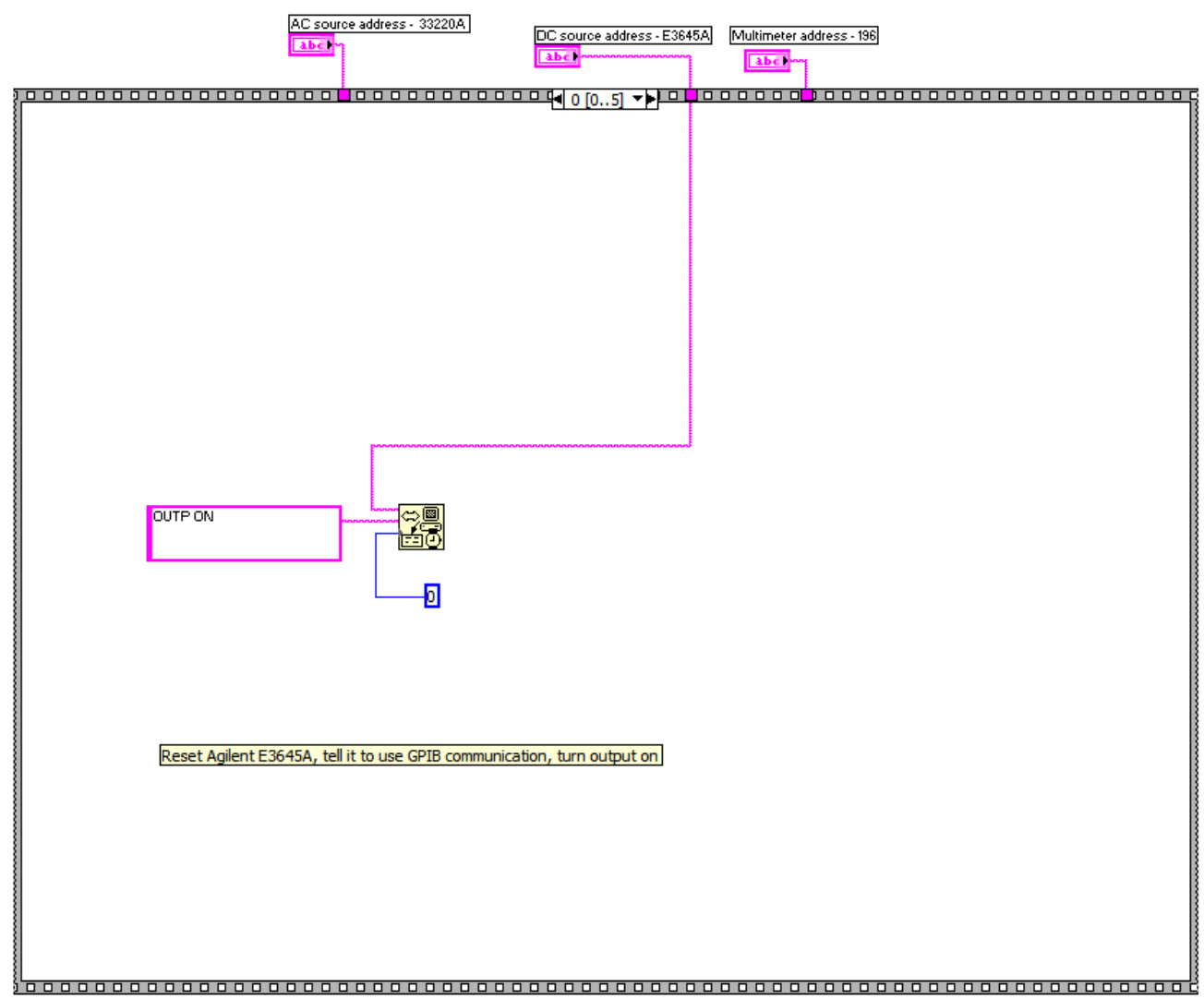



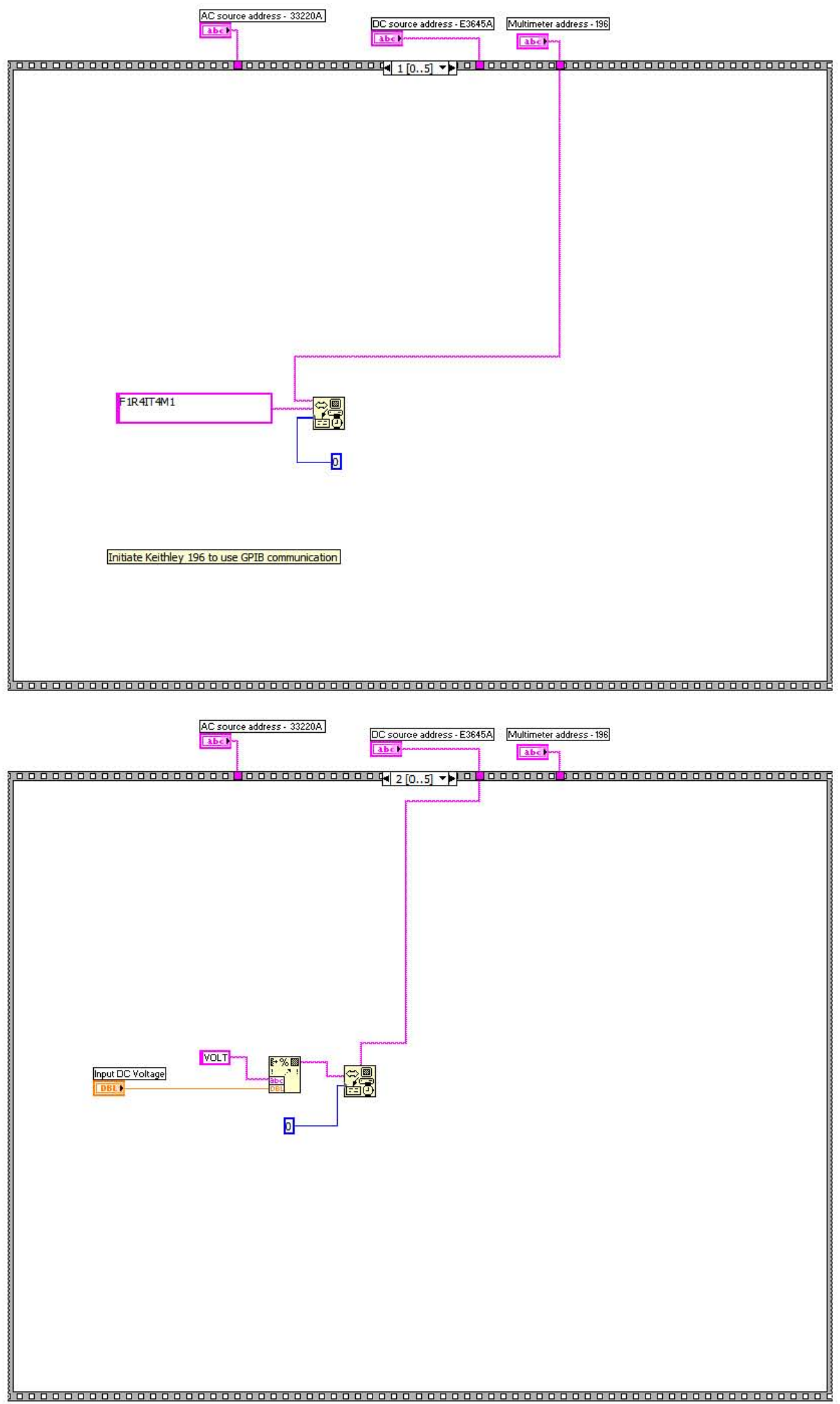


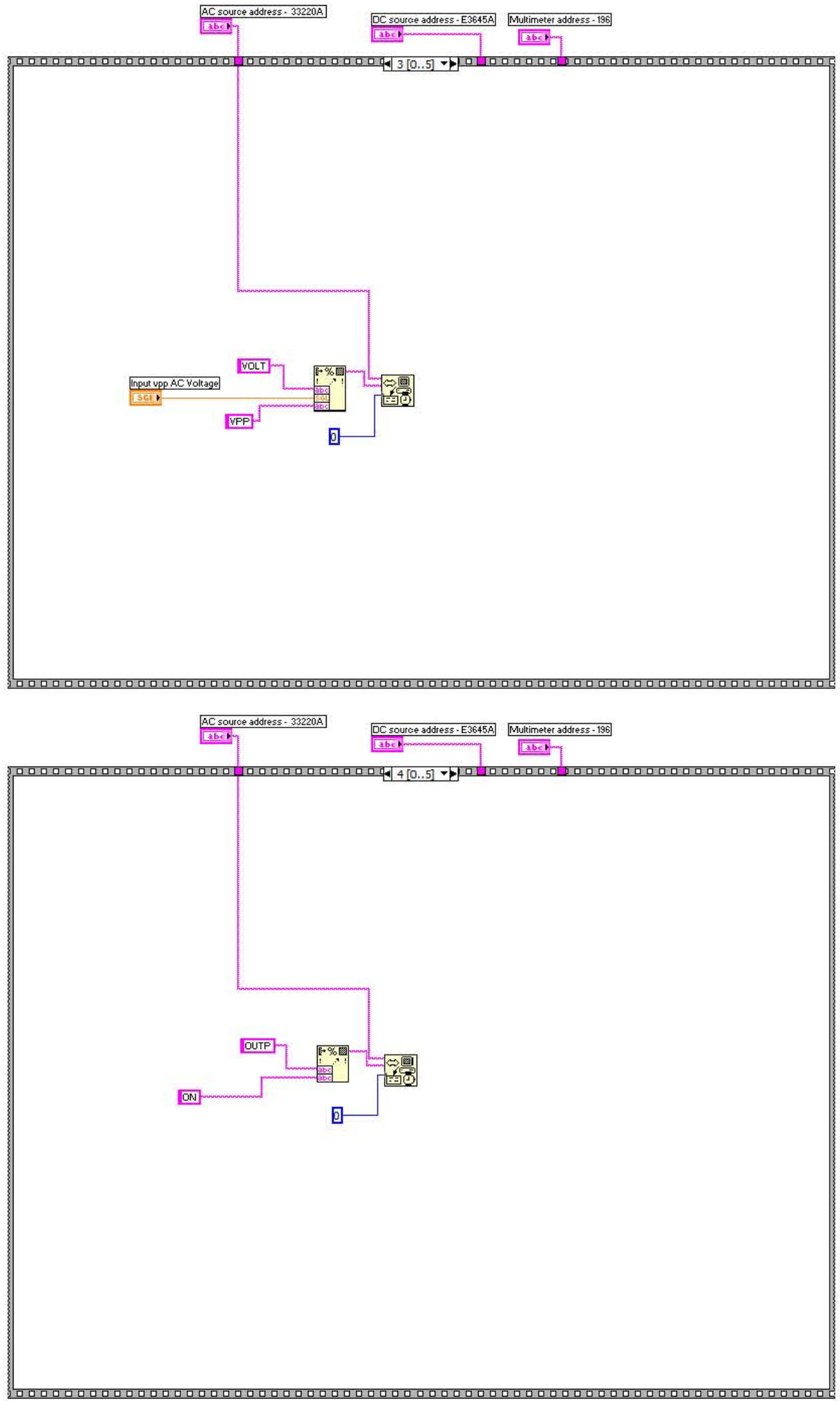




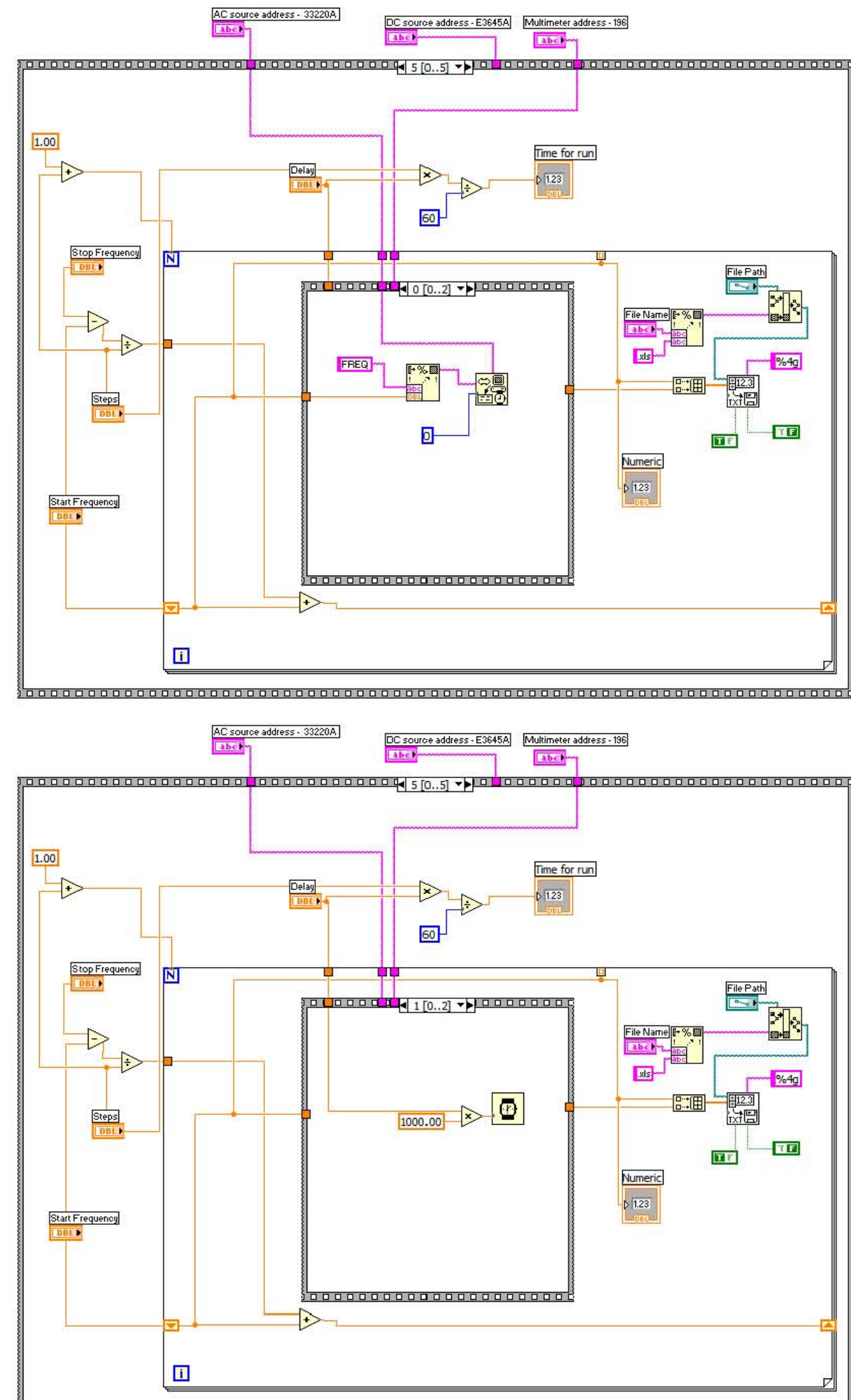

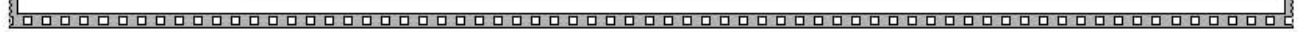




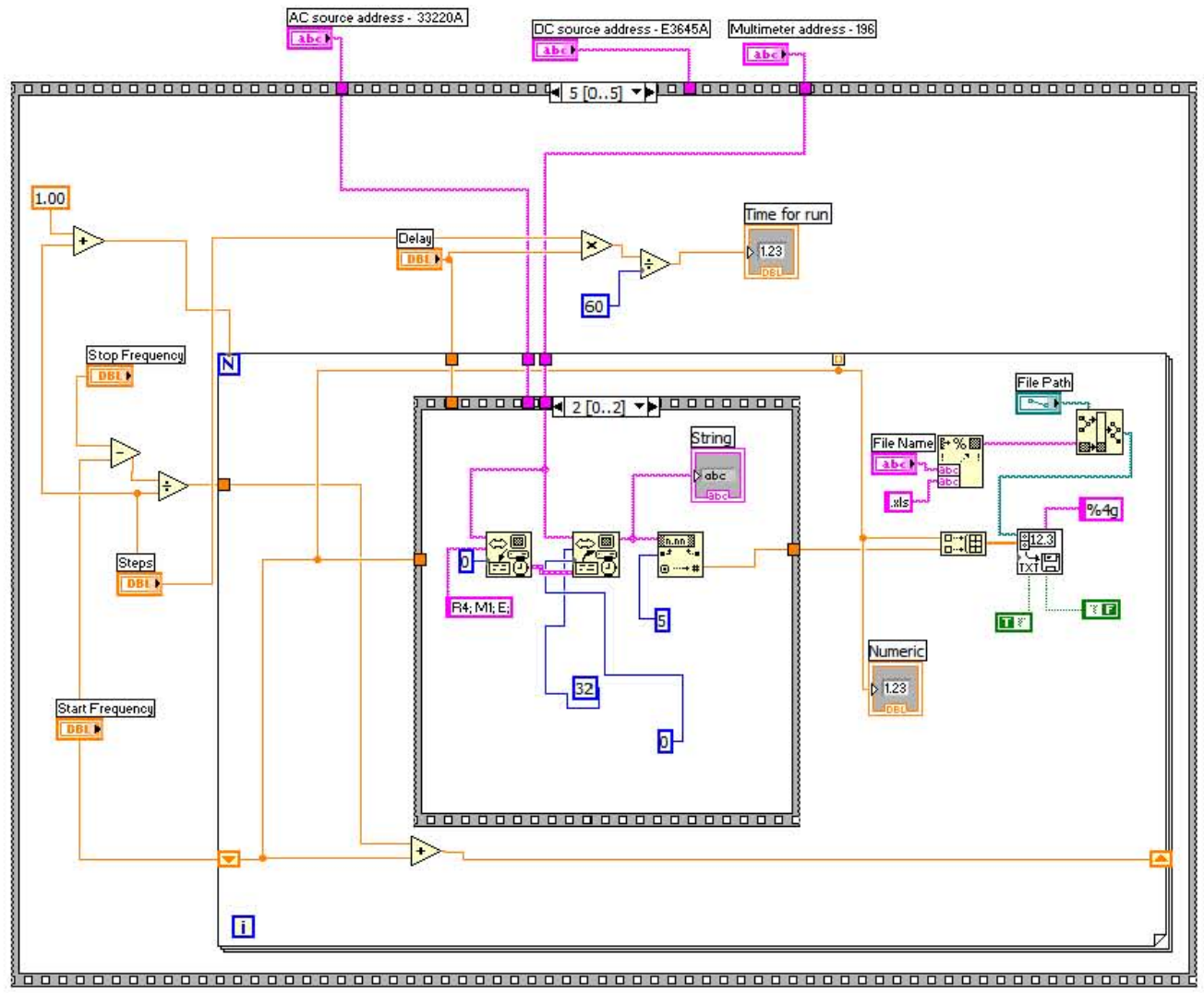




\section{APPENDIX B - MICROCANTILEVER METROLOGY}

The following spreadsheet contains the metrology measurements for each

microcantilever available on the seven fabricated devices. It also summarizes the resonant

frequency, Q factor, static sensitivity, and dynamic sensitivity for each microcantilever.

The summary at the end contains the mean and standard deviation for each parameter of

every microcantilever design.

\begin{tabular}{|c|c|c|c|c|c|c|c|c|c|c|}
\hline Beam & 1 & 2 & 3 & 4 & 5 & 6 & 7 & 8 & 9 & 10 \\
\hline \multicolumn{11}{|l|}{ Device 1} \\
\hline \multirow{4}{*}{$\begin{array}{c}\text { Width } \\
\text { Leg Length } \\
\text { Offset } \\
\text { Beam Length }\end{array}$} & 1.7 & 1.6 & 1.43 & 1.31 & 1.47 & 1.53 & 1.403 & 1.39 & 1.22 & 1.28 \\
\hline & 3.59 & 17.5 & 17.5 & 16.54 & 18.27 & 18.16 & 18.7 & 8.93 & 8.82 & 7.67 \\
\hline & 0 & 1 & 2 & 3.98 & 1.12 & 1.2 & 1.26 & 1.06 & 2.04 & 3.95 \\
\hline & 121.3 & 121 & 121.3 & 121 & 121.1 & 104 & 142.8 & 121.4 & 121.5 & 121.5 \\
\hline \multirow{4}{*}{$\begin{array}{c}\text { Res. Frequency } \\
\text { Q factor (freq/width) } \\
\text { Static Sens. }(\Omega / \mu \mathrm{m}) \\
\text { Dynamic Sens. }(\Omega / \mu \mathrm{m})\end{array}$} & & & 57500 & & 51865 & & 45862 & 40175 & 40877 & 50750 \\
\hline & & & 7666.6 & & 1383.1 & & 13103.4 & 11478.5 & 2043.8 & 2602.5 \\
\hline & & 2.55 & 1.6 & 2 & 1.2 & 6 & 5.55 & & 1.9 & 7 \\
\hline & & & 5.332 & & & & 19.519 & 6.004 & 2.816 & 15.115 \\
\hline \multicolumn{11}{|l|}{ Device 2} \\
\hline \multirow{4}{*}{$\begin{array}{c}\text { Width } \\
\text { Leg Length } \\
\text { Offset } \\
\text { Beam Length }\end{array}$} & 1.57 & 1.57 & 1.55 & 1.33 & 1.25 & 1.87 & 1.734 & 1.549 & 1.475 & 1.385 \\
\hline & 3.9 & 18.01 & 17.14 & 17.03 & 17.64 & 18.1 & 18.34 & 9.116 & 8.61 & 7.701 \\
\hline & 0 & 0.82 & 1.68 & 3.62 & 0.715 & 1.202 & 1.278 & 1.073 & 2.09 & 3.878 \\
\hline & 121.7 & 122.3 & 122 & 122.4 & 122.1 & 105.1 & 142.9 & 122.3 & 122.2 & 122.2 \\
\hline \multirow{3}{*}{$\begin{array}{c}\text { Res. Frequency } \\
\text { Q factor (freq/width) } \\
\text { Dynamic Sens. }(\Omega / \mu \mathrm{m})\end{array}$} & & & 54799 & 55947 & 52416 & & & & 51766 & \\
\hline & & & 3535.4 & 3390.7 & 2329.6 & & & & 1437.9 & \\
\hline & & & 6.316 & 6.628 & & & & & 3.901 & \\
\hline \multicolumn{11}{|l|}{ Device 3} \\
\hline \multirow{4}{*}{$\begin{array}{c}\text { Width } \\
\text { Leg Length } \\
\text { Offset } \\
\text { Beam Length }\end{array}$} & 1.234 & 1.095 & 1.041 & 1.03 & 1.159 & 1.2 & 1.06 & 0.953 & 1.03 & 1.05 \\
\hline & 4.036 & 18.35 & 17.82 & 16.95 & 18.53 & 18.4 & 18.37 & 9.251 & 8.9 & 7.833 \\
\hline & 0 & 0.98 & 1.851 & 3.778 & 0.95 & 1.043 & 1.06 & 0.95 & 2.054 & 3.926 \\
\hline & 121.8 & 121.9 & 121.9 & 121.6 & 121.8 & 104.3 & 143.3 & 122.2 & 121.8 & 122.2 \\
\hline \multirow{4}{*}{$\begin{array}{c}\text { Res. Frequency } \\
\text { Q factor (freq/width) } \\
\text { Static Sens. }(\Omega / \mu \mathrm{m}) \\
\text { Dynamic Sens. }(\Omega / \mu \mathrm{m})\end{array}$} & & 52298 & 46536 & 50658 & 48054 & & 39396 & 44972 & 42331 & \\
\hline & & 1743.2 & 3722.8 & 3752.4 & 2745.9 & & 2073.4 & 1499.0 & 1801.3 & \\
\hline & & 3.25 & 3.8125 & 2.15 & & 7.15 & 3.65 & 1.5 & 5.75 & 0.75 \\
\hline & & 12.817 & & & 3.294 & & 13.934 & 1.579 & 0.625 & \\
\hline \multicolumn{11}{|l|}{ Device 4} \\
\hline \multirow{4}{*}{$\begin{array}{c}\text { Width } \\
\text { Leg Length } \\
\text { Offset } \\
\text { Beam Length } \\
\end{array}$} & 1.424 & 1.26 & 1.06 & 1.14 & 1.21 & 1.184 & 1.16 & 1.025 & 1.041 & 1.081 \\
\hline & 3.917 & 18.18 & 17.05 & 17.09 & 18.31 & 18.22 & 18.4 & 9.045 & 8.735 & 7.753 \\
\hline & 0 & 0.96 & 1.78 & 3.63 & 0.81 & 1.093 & 1.099 & 1.085 & 2.025 & 3.759 \\
\hline & 120.9 & 121.4 & 121.6 & 121.4 & 121.9 & 104 & 142.1 & 122.1 & 121.6 & 121.6 \\
\hline \\
\hline \multicolumn{11}{|l|}{$\mathrm{Q}$ factor (freq/width) } \\
\hline \multicolumn{11}{|l|}{ Device 5} \\
\hline \multirow{4}{*}{$\begin{array}{c}\text { Width } \\
\text { Leg Length } \\
\text { Offset } \\
\text { Beam Length }\end{array}$} & & & 1.025 & & 1.293 & & & 1.025 & 1.2 & 1.147 \\
\hline & & & 17.69 & & 18.39 & & & 9.04 & 8.69 & 7.745 \\
\hline & & & 1.858 & & 0.86 & & & 0.96 & 1.943 & 3.81 \\
\hline & & & 121.2 & & 121.2 & & & 121.8 & 121.7 & 121.7 \\
\hline \multirow{2}{*}{$\begin{array}{c}\text { Res. Frequency } \\
\text { Q factor (freq/width) }\end{array}$} & & & 49266 & & & & & & 44735 & \\
\hline & & & 1642.2 & & & & & & 1491.1 & \\
\hline
\end{tabular}




\begin{tabular}{|c|c|c|c|c|c|c|c|c|c|c|}
\hline \multicolumn{11}{|l|}{ Device 6} \\
\hline \multicolumn{11}{|l|}{ Width } \\
\hline \multicolumn{11}{|l|}{ Leg Length } \\
\hline \multicolumn{11}{|l|}{ Offset } \\
\hline \multicolumn{11}{|l|}{ Beam Length } \\
\hline \multirow{2}{*}{$\begin{array}{l}\text { Res. Frequency } \\
\text { factor (freq/width) }\end{array}$} & & & & & & 64522 & & & & \\
\hline & & & & & & 4963.2 & & & & \\
\hline \multicolumn{11}{|l|}{ Device 7} \\
\hline \multirow{4}{*}{$\begin{array}{c}\text { Width } \\
\text { Leg Length } \\
\text { Offset } \\
\text { Beam Length }\end{array}$} & 0.958 & 1.033 & 1.059 & 1.2 & 1.284 & 1.082 & 1.132 & 1.169 & 1.111 & 1.136 \\
\hline & 4.2 & 18.37 & 17.89 & 16.99 & 18.04 & 18.59 & 18.48 & 9.095 & 8.709 & 7.733 \\
\hline & 0 & 0.98 & 1.854 & 3.81 & 0.96 & 1.082 & 0.94 & 0.89 & 1.987 & 3.757 \\
\hline & 122.3 & 122.7 & 122.1 & 122.1 & 121.9 & 104.9 & 143.2 & 122.9 & 122.2 & 122.6 \\
\hline \multirow{4}{*}{$\begin{array}{c}\text { Res. Frequency } \\
\text { Q factor (freq/width) } \\
\text { Static Sens. }(\Omega / \mu \mathrm{m}) \\
\text { Dynamic Sens. }(\Omega / \mu \mathrm{m})\end{array}$} & & 49392 & & 56048 & & 61190 & 35613 & 44209 & 44241 & 42124 \\
\hline & & 3087 & & 2802.4 & & 3399.4 & 2158.3 & 2763.0 & 1966.2 & 2632.7 \\
\hline & & & & & & 4.7 & 1.75 & 11.8125 & 0.6 & \\
\hline & & & & 0.0521 & & & & 1.6729 & & \\
\hline \multicolumn{11}{|l|}{ Averages } \\
\hline \multirow{3}{*}{$\begin{array}{c}\text { Avg Width } \\
\text { Desired Width } \\
\text { Avg Leg Length }\end{array}$} & 1.3772 & 1.3116 & 1.1941 & 1.202 & 1.2776 & 1.3732 & 1.2978 & 1.1851 & 1.1795 & 1.1798 \\
\hline & 1.1 & 1.1 & 1.1 & 1.1 & 1.3 & 1.1 & 1.1 & 1.1 & 1.1 & 1.1 \\
\hline & 3.9286 & 18.082 & 17.515 & 16.92 & 18.196 & 18.294 & 18.458 & 9.0795 & 8.744 & 7.7391 \\
\hline \multirow{2}{*}{$\begin{array}{l}\text { Desired Leg Length } \\
\text { Avg Offset }\end{array}$} & 4.5 & 20 & 19.5 & 18.5 & 20 & 20 & 20 & 10 & 9.5 & 8.5 \\
\hline & 0 & 0.948 & 1.8371 & 3.7636 & 0.9025 & 1.124 & 1.1274 & 1.003 & 2.0231 & 3.8466 \\
\hline Desired Offset & 0 & 1 & 2 & 4 & 1 & 1 & 1 & 1 & 2 & 4 \\
\hline \multirow{6}{*}{$\begin{array}{c}\text { Avg Beam Length } \\
\text { Desired Beam Length } \\
\text { Avg Res. Frequency } \\
\text { Avg Q Factor } \\
\text { Avg. Static Sens. } \\
\text { Avg. Dynamic Sens. }\end{array}$} & 121.6 & 121.86 & 121.68 & 121.7 & 121.66 & 104.46 & 142.86 & 122.11 & 121.83 & 121.96 \\
\hline & 128 & 128 & 128 & 128 & 128 & 110 & 150 & 128 & 128 & 128 \\
\hline & 0 & 50845 & 52025 & 54218 & 50778 & 62856 & 40290 & 43119 & 44790 & 46437 \\
\hline & 0 & 2415 & 2967 & 3315 & 2153 & 4181 & 2116 & 2131 & 1748 & 2618 \\
\hline & & 2.9 & 2.706 & 2.075 & 1.2 & 5.95 & 3.65 & 1.5 & 1.25 & 0.75 \\
\hline & & 12.817 & 5.824 & 3.340 & 3.294 & & 16.727 & 3.086 & 2.448 & \\
\hline \multicolumn{11}{|l|}{ Standard Deviations } \\
\hline \multirow{8}{*}{$\begin{array}{l}\text { Width Standard Dev. } \\
\text { Leg Length Std. Dev. } \\
\text { Offset Std Dev. } \\
\text { Beam Length Std Dev. } \\
\text { Res. Freq. Std. Dev. } \\
\text { Q Factor Std Dev. } \\
\text { Static Sens. Std Dev. } \\
\text { Dynamic Sens. Std Dev. }\end{array}$} & 0.291 & 0.263 & 0.23 & 0.123 & 0.106 & 0.324 & 0.275 & 0.236 & 0.164 & 0.127 \\
\hline & 0.224 & 0.356 & 0.35 & 0.218 & 0.316 & 0.199 & 0.144 & 0.105 & 0.102 & 0.055 \\
\hline & 0 & 0.072 & 0.105 & 0.148 & 0.140 & 0.072 & 0.142 & 0.080 & 0.051 & 0.083 \\
\hline & 0.529 & 0.680 & 0.376 & 0.556 & 0.413 & 0.512 & 0.472 & 0.503 & 0.301 & 0.432 \\
\hline & 0 & 2054.8 & 5013.7 & 3083.1 & 2375.3 & 2356.0 & 5182.7 & 2577.6 & 4193.0 & 6099.5 \\
\hline & 0 & 950.2 & 1151.0 & 479.5 & 698.4 & 1105.8 & 60.0 & 893.8 & 273.9 & 21.3 \\
\hline & & 0.495 & 1.564 & 0.106 & & 1.226 & 1.9 & & 0.919 & \\
\hline & & & 0.696 & 4.650 & & & 3.949 & 2.529 & 1.669 & \\
\hline
\end{tabular}




\section{APPENDIX C - STATIC DEFLECTION SENSITIVITY}

The following spreadsheet contains the deflection and resistance measurements for the tested microcantilevers during the static sensitivity experiment. The data has been plotted to the right of the measurements and the sensitivity was calculated.

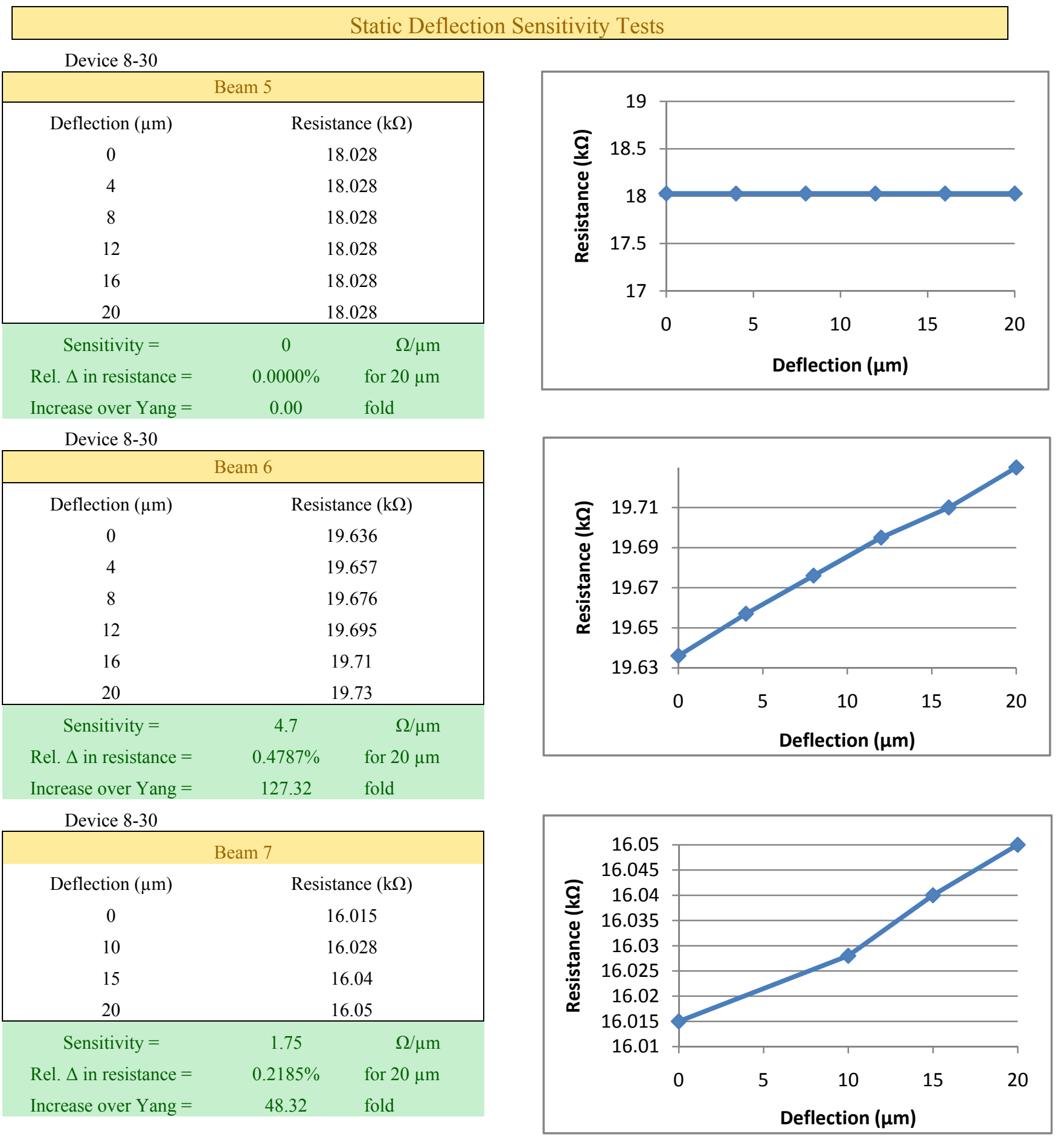


Device 8-30

\begin{tabular}{|cc|}
\hline \multicolumn{3}{|c|}{ Beam 8} \\
\hline Deflection $(\mu \mathrm{m})$ & Resistance $(\mathrm{k} \Omega)$ \\
0 & 11.681 \\
4 & 11.73 \\
8 & 11.744 \\
12 & 11.7 \\
16 & 11.87 \\
20 & 0 \\
\hline Sensitivity $=$ & 11.8125 \\
Rel. $\Delta$ in resistance $=$ & $1.6113 \%$ for $16 \mu \mathrm{m}$ \\
Increase over Yang $=$ & $499.39 \quad$ fold \\
\hline
\end{tabular}

Device 8-30

\begin{tabular}{|c|c|c|}
\hline \multicolumn{3}{|c|}{ Beam 9} \\
\hline Deflection $(\mu \mathrm{m})$ & \multicolumn{2}{|c|}{ Resistance $(\mathrm{k} \Omega)$} \\
\hline 0 & \multicolumn{2}{|c|}{14.729} \\
\hline 4 & \multicolumn{2}{|c|}{14.731} \\
\hline 8 & \multicolumn{2}{|c|}{14.733} \\
\hline 12 & \multicolumn{2}{|c|}{14.736} \\
\hline 16 & \multicolumn{2}{|c|}{14.738} \\
\hline 20 & \multicolumn{2}{|c|}{14.741} \\
\hline Sensitivity $=$ & 0.6 & $\Omega / \mu \mathrm{m}$ \\
\hline Rel. $\Delta$ in resistance $=$ & $0.0815 \%$ & for $20 \mu \mathrm{m}$ \\
\hline Increase over Yang = & 16.17 & fold \\
\hline
\end{tabular}

Device 7-24

\begin{tabular}{|c|c|c|}
\hline \multicolumn{3}{|c|}{ Beam 2} \\
\hline Deflection $(\mu \mathrm{m})$ & \multicolumn{2}{|c|}{ Resistance $(\mathrm{k} \Omega)$} \\
\hline 0 & \multicolumn{2}{|c|}{14.57} \\
\hline 4 & \multicolumn{2}{|c|}{14.575} \\
\hline 8 & \multicolumn{2}{|c|}{14.58} \\
\hline 12 & \multicolumn{2}{|c|}{14.59} \\
\hline 16 & \multicolumn{2}{|c|}{14.608} \\
\hline 20 & \multicolumn{2}{|c|}{14.621} \\
\hline Sensitivity $=$ & 2.55 & $\Omega / \mu \mathrm{m}$ \\
\hline Rel. $\Delta$ in resistance $=$ & $0.3500 \%$ & for $20 \mu \mathrm{m}$ \\
\hline Increase over Yang $=$ & 40.85 & fold \\
\hline
\end{tabular}
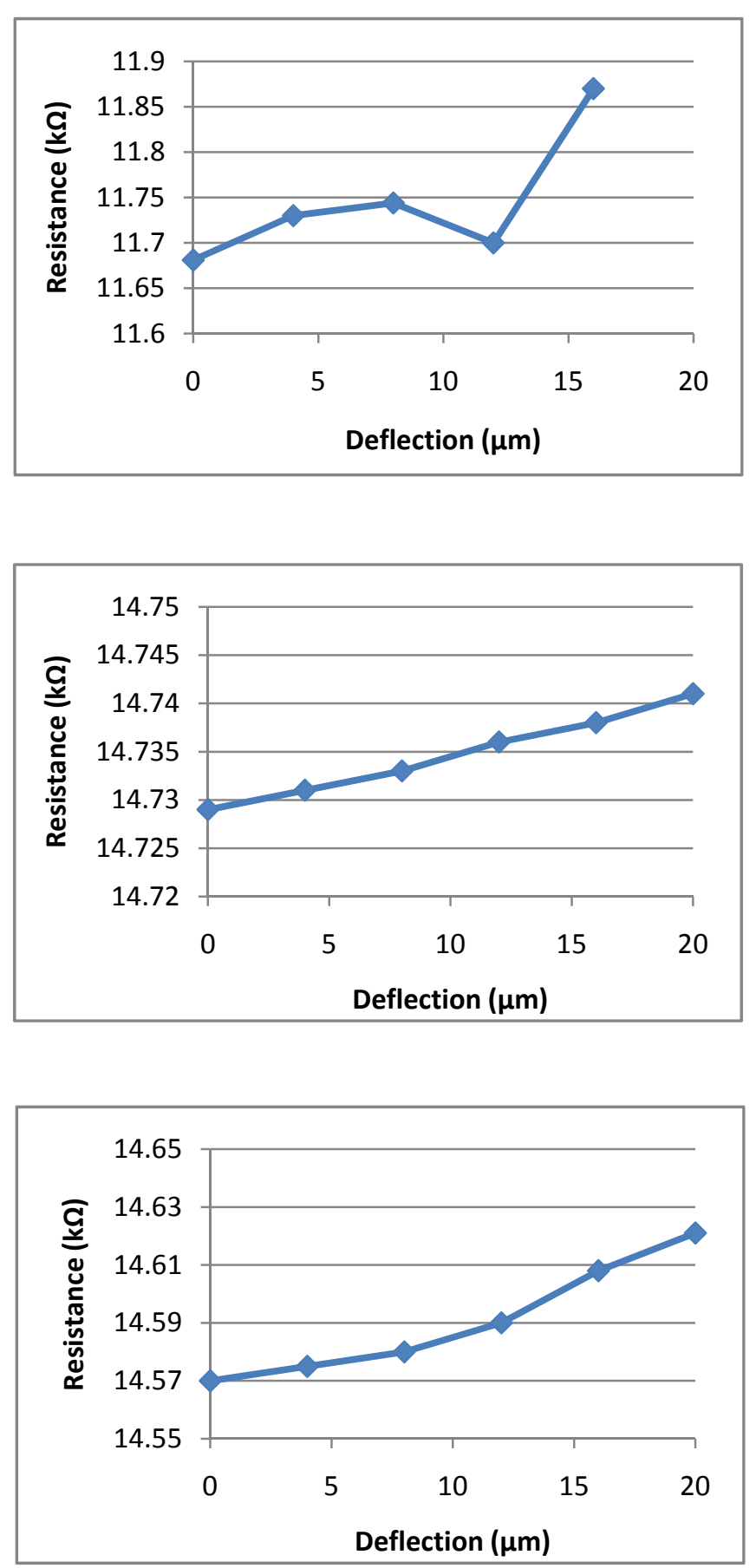
Device 7-24

\begin{tabular}{|c|c|c|}
\hline \multicolumn{3}{|c|}{ Beam 3} \\
\hline Deflection $(\mu \mathrm{m})$ & \multicolumn{2}{|c|}{ Resistance $(\mathrm{k} \Omega)$} \\
\hline 0 & \multicolumn{2}{|c|}{12.971} \\
\hline 4 & \multicolumn{2}{|c|}{12.982} \\
\hline 8 & \multicolumn{2}{|c|}{12.986} \\
\hline 12 & \multicolumn{2}{|c|}{12.99} \\
\hline 16 & \multicolumn{2}{|c|}{12.995} \\
\hline 20 & \multicolumn{2}{|c|}{13.003} \\
\hline Sensitivity $=$ & 1.6 & $\Omega / \mu \mathrm{m}$ \\
\hline Rel. $\Delta$ in resistance $=$ & $0.2467 \%$ & for $20 \mu \mathrm{m}$ \\
\hline Increase over Yang = & 100.96 & fold \\
\hline
\end{tabular}

Device 7-24

\begin{tabular}{|c|c|c|}
\hline \multicolumn{3}{|c|}{ Beam 4} \\
\hline Deflection $(\mu \mathrm{m})$ & \multicolumn{2}{|c|}{ Resistance $(\mathrm{k} \Omega)$} \\
\hline 0 & \multicolumn{2}{|c|}{14.605} \\
\hline 4 & \multicolumn{2}{|c|}{14.609} \\
\hline 8 & \multicolumn{2}{|c|}{14.614} \\
\hline 12 & \multicolumn{2}{|c|}{14.623} \\
\hline 16 & \multicolumn{2}{|c|}{14.635} \\
\hline 20 & \multicolumn{2}{|c|}{14.645} \\
\hline Sensitivity $=$ & 2 & $\Omega / \mu \mathrm{m}$ \\
\hline Rel. $\Delta$ in resistance $=$ & $0.2739 \%$ & for $20 \mu \mathrm{m}$ \\
\hline Increase over Yang = & 32.60 & fold \\
\hline
\end{tabular}

Device 7-24

\begin{tabular}{|c|c|c|}
\hline \multicolumn{3}{|c|}{ Beam 5} \\
\hline Deflection $(\mu \mathrm{m})$ & \multicolumn{2}{|c|}{ Resistance $(\mathrm{k} \Omega)$} \\
\hline 0 & \multicolumn{2}{|c|}{17.716} \\
\hline 4 & \multicolumn{2}{|c|}{17.722} \\
\hline 8 & \multicolumn{2}{|c|}{17.726} \\
\hline 12 & \multicolumn{2}{|c|}{17.729} \\
\hline 16 & \multicolumn{2}{|c|}{17.734} \\
\hline 20 & \multicolumn{2}{|c|}{17.74} \\
\hline Sensitivity $=$ & 1.2 & $\Omega / \mu \mathrm{m}$ \\
\hline Rel. $\Delta$ in resistance $=$ & $0.1355 \%$ & for $20 \mu \mathrm{m}$ \\
\hline Increase over Yang = & 40.32 & fold \\
\hline
\end{tabular}
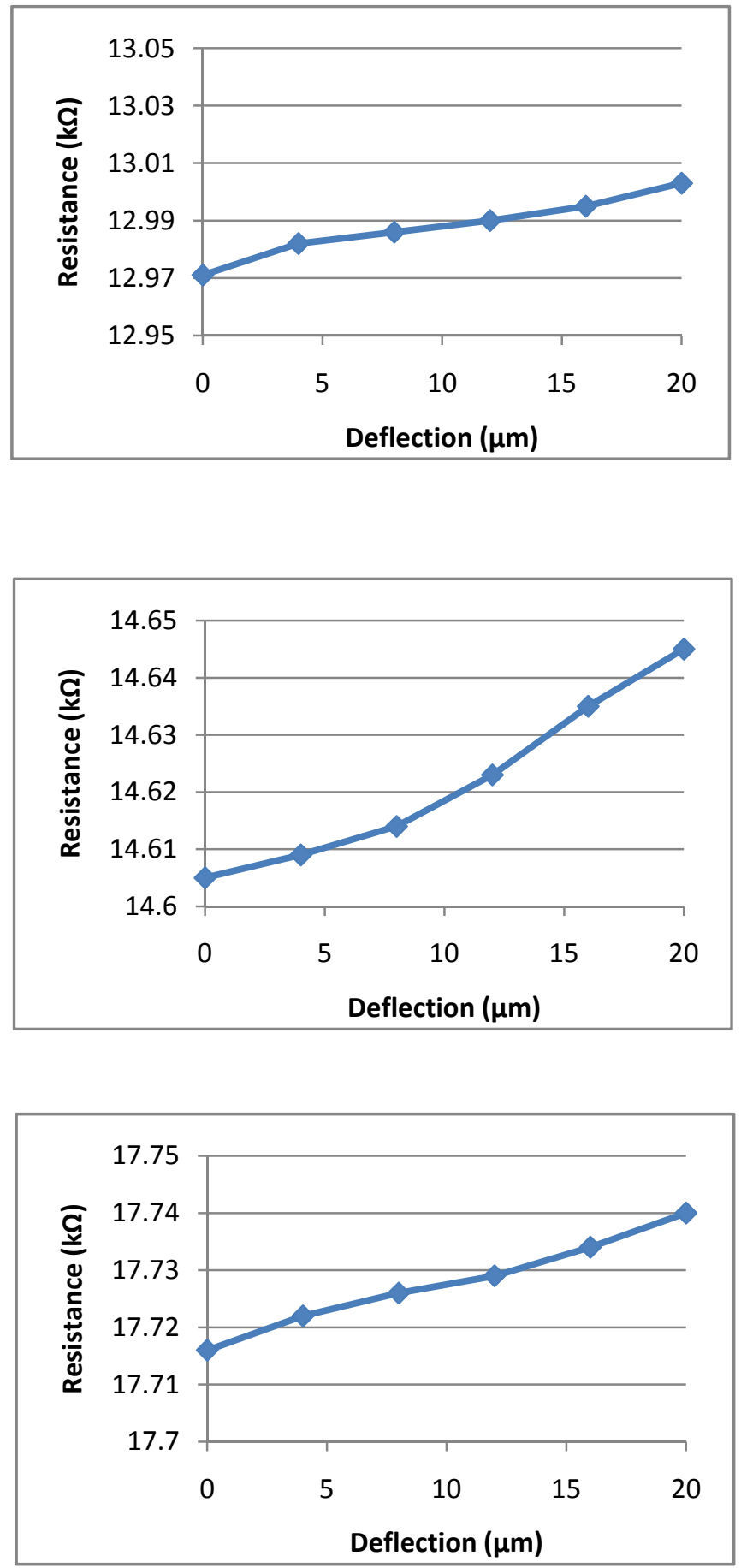
Device 7-24

\begin{tabular}{|c|c|c|}
\hline \multicolumn{3}{|c|}{ Beam 6} \\
\hline Deflection $(\mu \mathrm{m})$ & \multicolumn{2}{|c|}{ Resistance $(\mathrm{k} \Omega)$} \\
\hline 0 & \multicolumn{2}{|c|}{17.565} \\
\hline 4 & \multicolumn{2}{|c|}{17.588} \\
\hline 8 & \multicolumn{2}{|c|}{17.611} \\
\hline 12 & \multicolumn{2}{|c|}{17.64} \\
\hline 16 & \multicolumn{2}{|c|}{17.658} \\
\hline 20 & \multicolumn{2}{|c|}{17.685} \\
\hline Sensitivity $=$ & 6 & $\Omega / \mu \mathrm{m}$ \\
\hline Rel. $\Delta$ in resistance $=$ & $0.6832 \%$ & for $20 \mu \mathrm{m}$ \\
\hline Increase over Yang = & 155.88 & fold \\
\hline \multicolumn{3}{|l|}{ Device 7-24 } \\
\hline \multicolumn{3}{|c|}{ Beam 7} \\
\hline Deflection $(\mu \mathrm{m})$ & \multicolumn{2}{|c|}{ Resistance $(\mathrm{k} \Omega$ ) } \\
\hline 0 & \multicolumn{2}{|c|}{15.971} \\
\hline 4 & \multicolumn{2}{|c|}{16} \\
\hline 8 & \multicolumn{2}{|c|}{16.022} \\
\hline 12 & \multicolumn{2}{|c|}{16.041} \\
\hline 16 & \multicolumn{2}{|c|}{16.063} \\
\hline 20 & \multicolumn{2}{|c|}{16.082} \\
\hline Sensitivity $=$ & 5.55 & $\Omega / \mu \mathrm{m}$ \\
\hline Rel. $\Delta$ in resistance $=$ & $0.6950 \%$ & for $20 \mu \mathrm{m}$ \\
\hline Increase over Yang = & 216.17 & fold \\
\hline \multicolumn{3}{|l|}{ Device 7-24 } \\
\hline \multicolumn{3}{|c|}{ Beam 9} \\
\hline Deflection $(\mu \mathrm{m})$ & \multicolumn{2}{|c|}{ Resistance $(\mathrm{k} \Omega)$} \\
\hline 0 & \multicolumn{2}{|c|}{14.854} \\
\hline 4 & \multicolumn{2}{|c|}{14.863} \\
\hline 8 & \multicolumn{2}{|c|}{14.87} \\
\hline 12 & \multicolumn{2}{|c|}{14.877} \\
\hline 16 & \multicolumn{2}{|c|}{14.885} \\
\hline 20 & \multicolumn{2}{|c|}{14.892} \\
\hline Sensitivity $=$ & 1.9 & $\Omega / \mu \mathrm{m}$ \\
\hline Rel. $\Delta$ in resistance $=$ & $0.2558 \%$ & for $20 \mu \mathrm{m}$ \\
\hline Increase over Yang = & 72.13 & fold \\
\hline
\end{tabular}
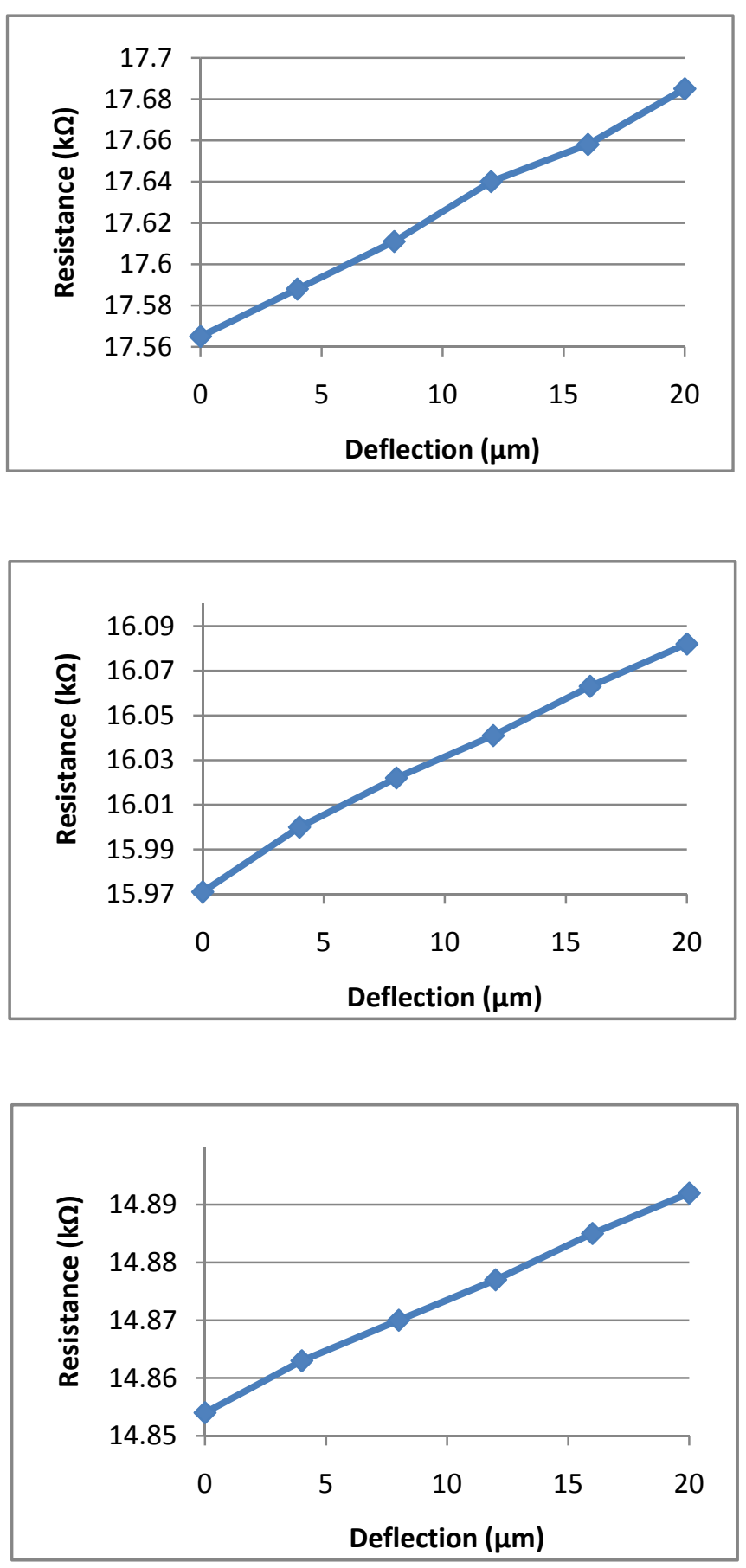
Device 7-24

\begin{tabular}{|c|c|c|}
\hline \multicolumn{3}{|c|}{ Beam 10} \\
\hline Deflection $(\mu \mathrm{m})$ & \multicolumn{2}{|c|}{ Resistance $(\mathrm{k} \Omega)$} \\
\hline 0 & \multicolumn{2}{|c|}{17.357} \\
\hline 4 & \multicolumn{2}{|c|}{17.357} \\
\hline 8 & \multicolumn{2}{|c|}{17.392} \\
\hline 12 & \multicolumn{2}{|c|}{17.441} \\
\hline 16 & & \\
\hline 20 & & \\
\hline Sensitivity $=$ & 7 & $\Omega / \mu \mathrm{m}$ \\
\hline Rel. $\Delta$ in resistance $=$ & $0.4840 \%$ & for $20 \mu \mathrm{m}$ \\
\hline Increase over Yang = & 0.00 & fold \\
\hline
\end{tabular}

Device 7-30.2

\begin{tabular}{|c|c|c|}
\hline \multicolumn{3}{|c|}{ Beam 2} \\
\hline Deflection $(\mu \mathrm{m})$ & \multicolumn{2}{|c|}{ Resistance $(\mathrm{k} \Omega)$} \\
\hline 0 & \multicolumn{2}{|c|}{16.893} \\
\hline 4 & \multicolumn{2}{|c|}{16.88} \\
\hline 8 & \multicolumn{2}{|c|}{16.867} \\
\hline 12 & \multicolumn{2}{|c|}{16.858} \\
\hline 16 & \multicolumn{2}{|c|}{16.831} \\
\hline 20 & \multicolumn{2}{|c|}{16.828} \\
\hline Sensitivity $=$ & -3.25 & $\Omega / \mu \mathrm{m}$ \\
\hline Rel. $\Delta$ in resistance $=$ & $-0.3848 \%$ & for $20 \mu \mathrm{m}$ \\
\hline Increase over Yang $=$ & -91.61 & fold \\
\hline
\end{tabular}

Device 7-30.2

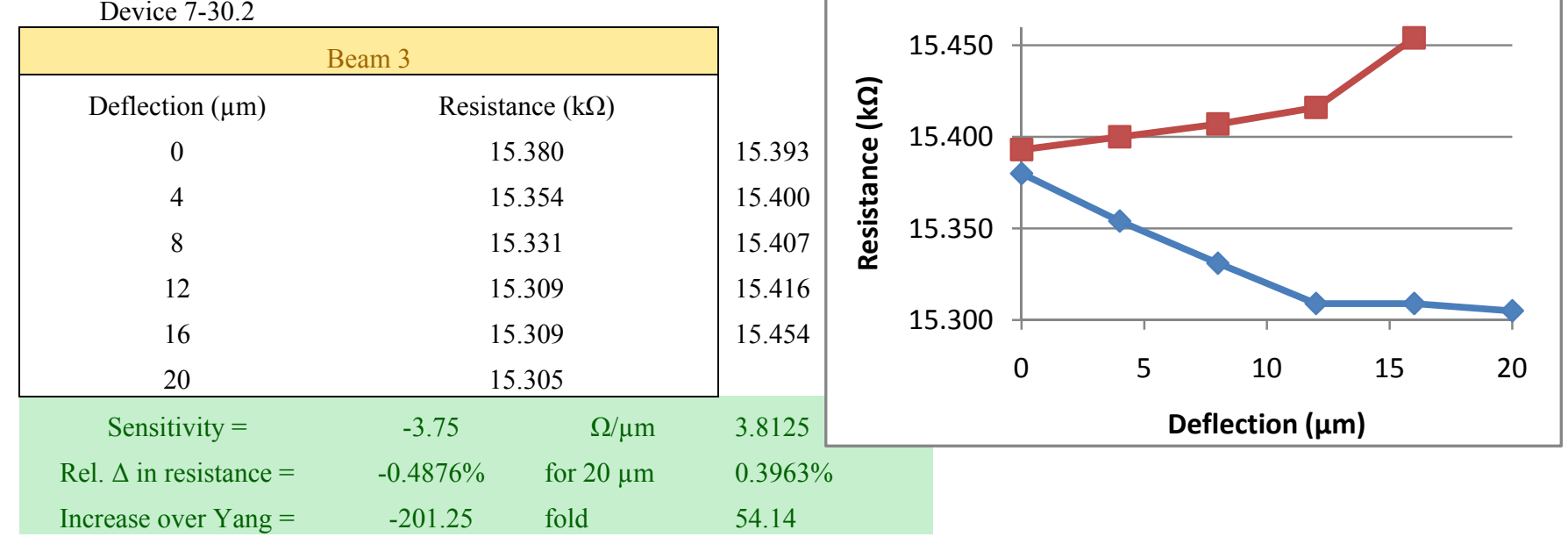


Device 7-30.2

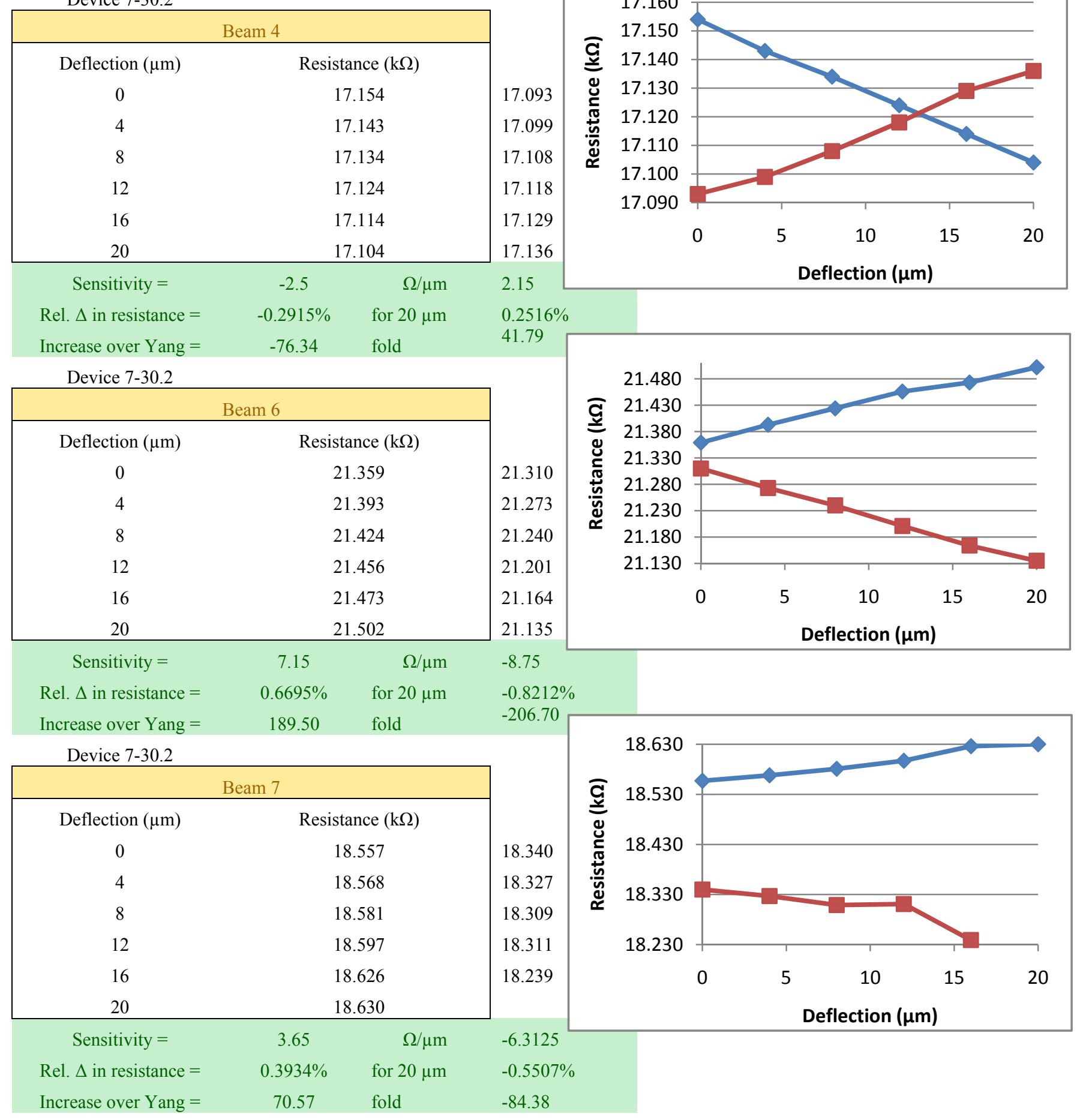


Device 7-30.2

\begin{tabular}{|c|c|c|}
\hline \multicolumn{3}{|c|}{ Beam 8} \\
\hline Deflection $(\mu \mathrm{m})$ & \multicolumn{2}{|c|}{ Resistance $(\mathrm{k} \Omega)$} \\
\hline 0 & \multicolumn{2}{|c|}{14.185} \\
\hline 4 & \multicolumn{2}{|c|}{14.185} \\
\hline 8 & \multicolumn{2}{|c|}{14.188} \\
\hline 12 & \multicolumn{2}{|c|}{14.193} \\
\hline 16 & \multicolumn{2}{|c|}{14.205} \\
\hline 20 & \multicolumn{2}{|c|}{14.215} \\
\hline Sensitivity $=$ & 1.5 & $\Omega / \mu \mathrm{m}$ \\
\hline Rel. $\Delta$ in resistance $=$ & $0.2115 \%$ & for $20 \mu \mathrm{m}$ \\
\hline Increase over Yang = & 0.00 & fold \\
\hline
\end{tabular}

Device 7-30.2

\begin{tabular}{|c|c|c|}
\hline \multicolumn{3}{|c|}{ Beam 9} \\
\hline Deflection $(\mu \mathrm{m})$ & \multicolumn{2}{|c|}{ Resistance $(\mathrm{k} \Omega)$} \\
\hline 0 & \multicolumn{2}{|c|}{15.742} \\
\hline 4 & \multicolumn{2}{|c|}{15.739} \\
\hline 8 & \multicolumn{2}{|c|}{15.737} \\
\hline 12 & \multicolumn{2}{|c|}{15.734} \\
\hline 16 & \multicolumn{2}{|c|}{15.730} \\
\hline 20 & \multicolumn{2}{|c|}{15.723} \\
\hline Sensitivity $=$ & -0.95 & $\Omega / \mu \mathrm{m}$ \\
\hline Rel. $\Delta$ in resistance $=$ & $-0.1207 \%$ & for $20 \mu \mathrm{m}$ \\
\hline Increase over Yang = & -22.69 & fold \\
\hline \multicolumn{3}{|l|}{ Device 7-30.2 } \\
\hline \multicolumn{3}{|c|}{ Beam 10} \\
\hline Deflection $(\mu \mathrm{m})$ & Resis & cee $(\mathrm{k} \Omega)$ \\
\hline 0 & & 140 \\
\hline 4 & & 142 \\
\hline 8 & & 142 \\
\hline 12 & & 138 \\
\hline 16 & & 128 \\
\hline 20 & & \\
\hline
\end{tabular}

Sensitivity $=$

Rel. $\Delta$ in resistance $=$ Increase over Yang =

\section{$-0.75$} $-0.0743 \%$ 14.75
15.788

15.797

15.805

15.823

15.880

\subsection{5}

$0.5827 \%$

67.86

15.936
15.906
15.907
15.912
15.933
15.924

$-0.6$

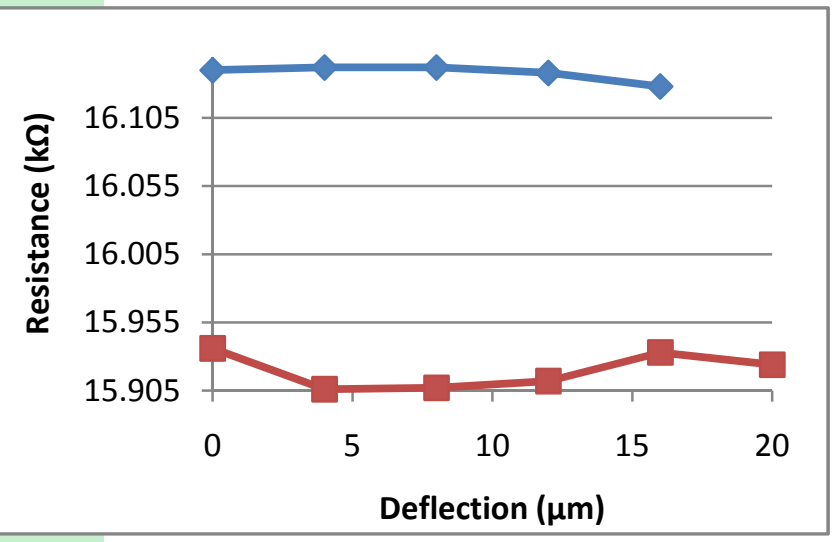

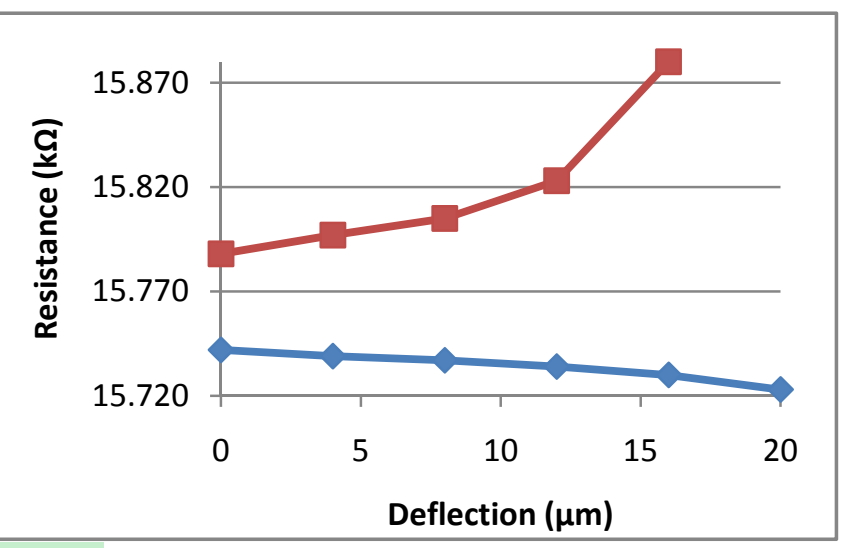

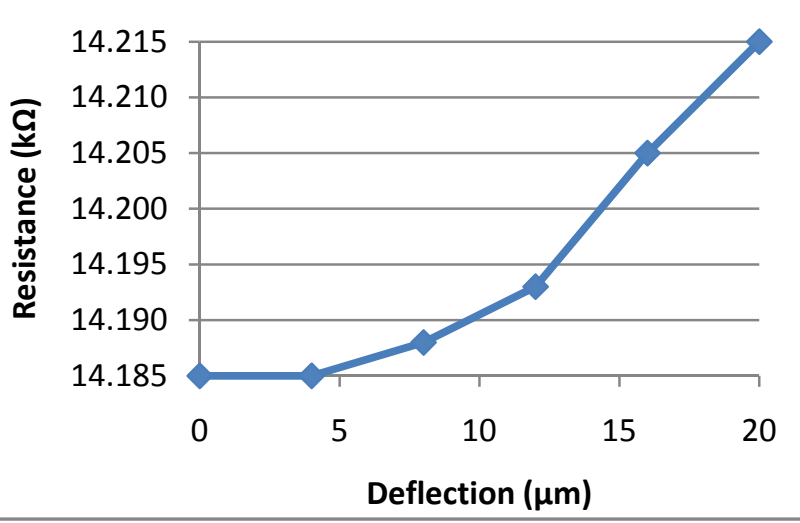




\section{APPENDIX D - DYNAMIC DEFLECTION SENSITIVITY}

The following spreadsheet contains the deflection and resistance measurements for the tested microcantilevers during the dynamic sensitivity experiment. The lock-in amplifier output has been plotted to the right of the measurements (x-axis is the cantilever frequency and y-axis is lock-in amp output in volts) and the sensitivity was calculated.

\section{Dynamic Sensitivity Tests}

Device 7-24

\begin{tabular}{|lcc|}
\hline \multicolumn{3}{|c|}{ Beam 3 } \\
\hline Maximum deflection $=$ & 8.07 & $\mu \mathrm{m}$ \\
Max voltage output $=$ & 0.042689873 & Volts \\
Min voltage output $=$ & 0.011988608 & Volts \\
Current in circuit $=$ & 0.000713429 & Amps \\
Initial resistance $=$ & 16.80419652 & $\Omega$ \\
Final resistance $=$ & 59.83755969 & $\Omega$ \\
Sensitivity $=$ & 5.332510925 & $\Omega / \mu \mathrm{m}$ \\
\hline
\end{tabular}

Device 7-24

\begin{tabular}{|lcc|}
\hline \multicolumn{3}{|c|}{ Beam 7} \\
\hline Maximum deflection $=$ & 7.0285 & $\mu \mathrm{m}$ \\
Max voltage output $=$ & 0.107902532 & Volts \\
Min voltage output $=$ & 0.010006329 & Volts \\
Current in circuit $=$ & 0.000713571 & Amps \\
Initial resistance $=$ & 14.02289253 & $\Omega$ \\
Final resistance $=$ & 151.2148549 & $\Omega$ \\
Sensitivity $=$ & 19.51938 & $\Omega / \mu \mathrm{m}$ \\
\hline
\end{tabular}

Device 7-24

\begin{tabular}{|lcc|}
\hline \multicolumn{3}{|c|}{ Beam 8} \\
\hline Maximum deflection $=$ & 8.595 & $\mu \mathrm{m}$ \\
Max voltage output $=$ & 0.039226582 & Volts \\
Min voltage output $=$ & 0.00236962 & Volts \\
Current in circuit $=$ & 0.000714116 & Amps \\
Initial resistance $=$ & 3.318254655 & $\Omega$ \\
Final resistance $=$ & 54.93023157 & $\Omega$ \\
Sensitivity $=$ & 6.004883876 & $\Omega / \mu \mathrm{m}$ \\
\hline
\end{tabular}
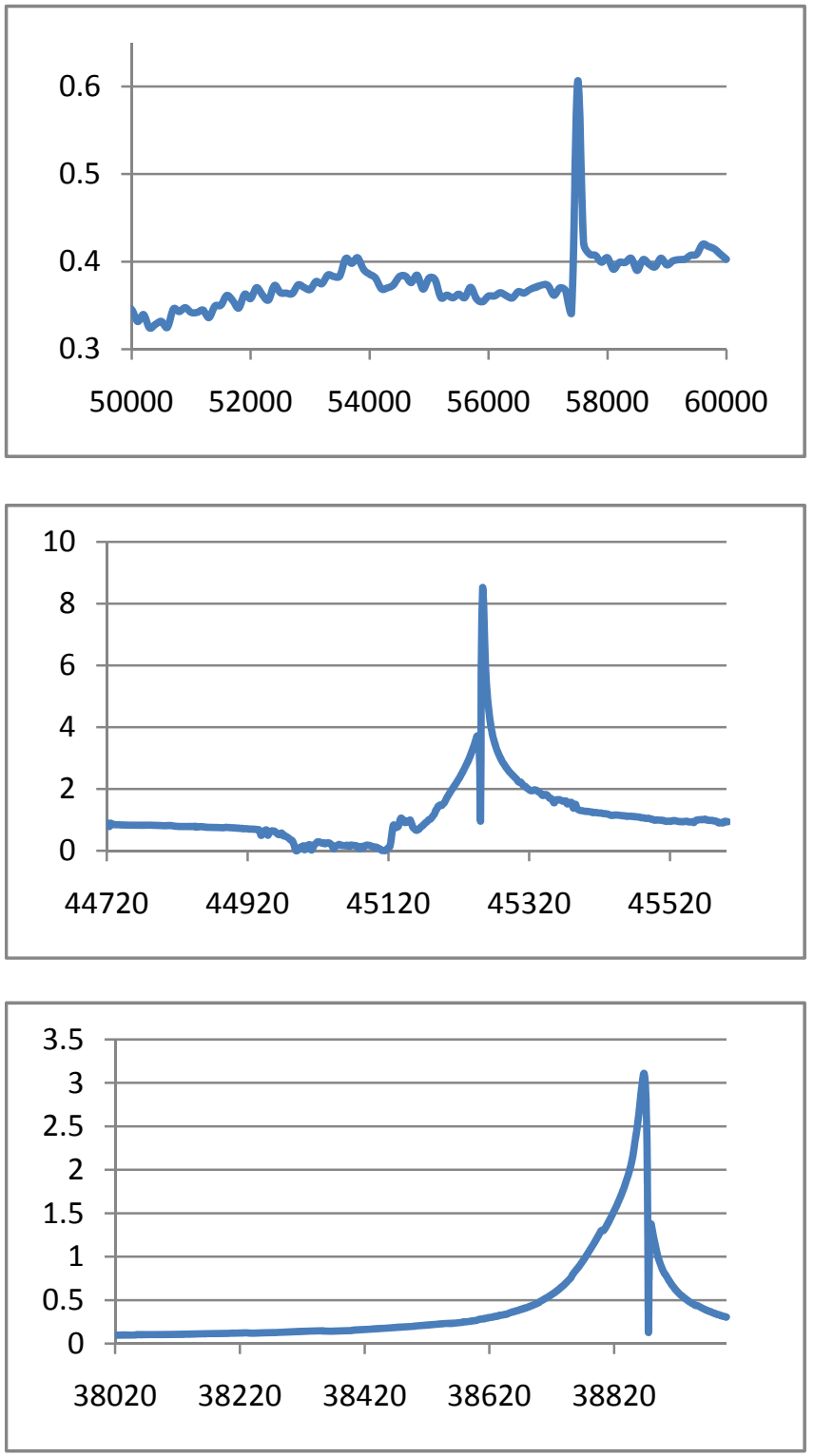
Device 7-24

\begin{tabular}{|lcc|}
\hline \multicolumn{3}{|c|}{ Beam 9} \\
\hline Maximum deflection $=$ & 7.81 & $\mu \mathrm{m}$ \\
Max voltage output $=$ & 1.310346924 & Volts \\
Min voltage output $=$ & 1.296670567 & Volts \\
Current in circuit $=$ & 0.000621666 & Amps \\
Initial resistance $=$ & 2085.798093 & $\Omega$ \\
Final resistance $=$ & 2107.797604 & $\Omega$ \\
Sensitivity $=$ & 2.816838812 & $\Omega / \mu \mathrm{m}$ \\
\hline
\end{tabular}

Device 7-24

\begin{tabular}{|lcc|}
\hline \multicolumn{3}{|c|}{ Beam 10} \\
\hline Maximum deflection $=$ & 5.9 & $\mu \mathrm{m}$ \\
Max voltage output $=$ & 0.439696203 & Volts \\
Min voltage output $=$ & 0.378405063 & Volts \\
Current in circuit $=$ & 0.000687257 & Amps \\
Initial resistance $=$ & 550.6021529 & $\Omega$ \\
Final resistance $=$ & 639.7844511 & $\Omega$ \\
Sensitivity $=$ & 15.11564377 & $\Omega / \mu \mathrm{m}$ \\
\hline
\end{tabular}

Device 7-30.1

\begin{tabular}{|lcc|}
\hline \multicolumn{3}{|c|}{ Beam 3 } \\
\hline Maximum deflection $=$ & 9.355 & $\mu \mathrm{m}$ \\
Max voltage output $=$ & 0.050737975 & Volts \\
Min voltage output $=$ & 0.008568354 & Volts \\
Current in circuit $=$ & 0.000713674 & Amps \\
Initial resistance $=$ & 12.00598335 & $\Omega$ \\
Final resistance $=$ & 71.09408048 & $\Omega$ \\
Sensitivity $=$ & 6.316204931 & $\Omega / \mu \mathrm{m}$ \\
\hline
\end{tabular}

Device 7-30.1

\begin{tabular}{|lcc|}
\hline \multicolumn{3}{|c|}{ Beam 4} \\
\hline Maximum deflection $=$ & 7.61 & $\mu \mathrm{m}$ \\
Max voltage output $=$ & 0.056325316 & Volts \\
Min voltage output $=$ & 0.02036962 & Volts \\
Current in circuit $=$ & 0.000712831 & Amps \\
Initial resistance $=$ & 28.57567592 & $\Omega$ \\
Final resistance $=$ & 79.01639644 & $\Omega$ \\
Sensitivity $=$ & 6.628215574 & $\Omega / \mu \mathrm{m}$ \\
\hline
\end{tabular}
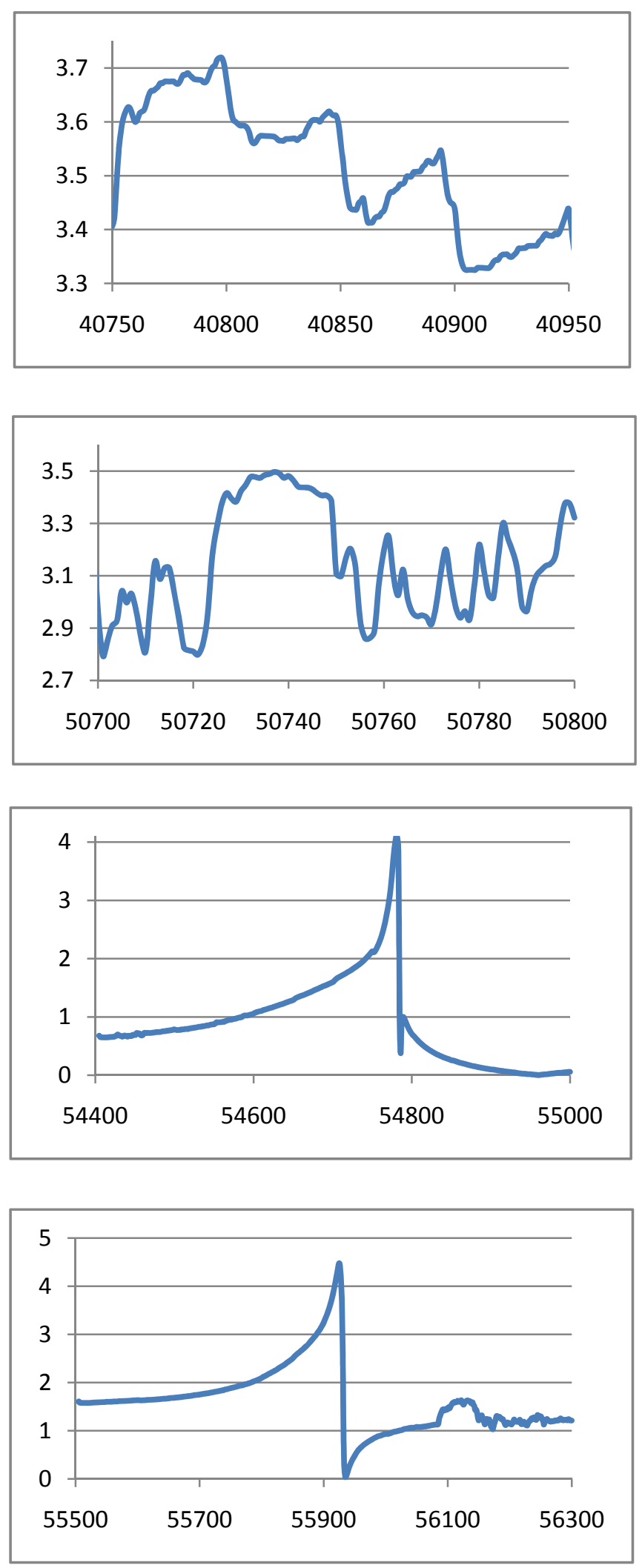
Device 7-30.1

\begin{tabular}{|lcc|}
\hline \multicolumn{3}{|c|}{ Beam 9} \\
\hline Maximum deflection $=$ & 8.2975 & $\mu \mathrm{m}$ \\
Max voltage output $=$ & 0.06575443 & Volts \\
Min voltage output $=$ & 0.04273038 & Volts \\
Current in circuit $=$ & 0.000711234 & Amps \\
Initial resistance $=$ & 60.07925257 & $\Omega$ \\
Final resistance $=$ & 92.45125023 & $\Omega$ \\
Sensitivity $=$ & 3.901415807 & $\Omega / \mu \mathrm{m}$ \\
\hline
\end{tabular}

Device 7-30.2

\begin{tabular}{|ccc|}
\hline \multicolumn{3}{|c|}{ Beam 2 } \\
\hline Maximum deflection \\
Max voltage output $=$ & 1.205076967 & Volts \\
Min voltage output $=$ & 1.137796891 & Volts \\
Current in circuit $=$ & 0.000633015 & Amps \\
Initial resistance $=$ & 1797.426247 & $\Omega$ \\
Final resistance $=$ & 1903.711452 & $\Omega$ \\
Sensitivity $=$ & 12.81702801 & $\Omega / \mu \mathrm{m}$ \\
\hline
\end{tabular}

Device 7-30.2

\begin{tabular}{|ccc|}
\hline \multicolumn{3}{|c|}{ Beam 5 } \\
\hline Maximum deflection \\
$=$ & 10.0355 & $\mu \mathrm{m}$ \\
Max voltage output $=$ & 0.028293723 & Volts \\
Min voltage output $=$ & 0.00469213 & Volts \\
Current in circuit $=$ & 0.000713951 & Amps \\
Initial resistance $=$ & 6.572065722 & $\Omega$ \\
Final resistance $=$ & 39.62980639 & $\Omega$ \\
Sensitivity $=$ & 3.294080083 & $\Omega / \mu \mathrm{m}$ \\
\hline
\end{tabular}

Device 7-30.2

\begin{tabular}{|ccc|}
\hline \multicolumn{3}{|c|}{ Beam 7 } \\
\hline Maximum deflection \\
Max voltage output $=$ & 0.392886076 & Volts \\
Min voltage output $=$ & 0.18764557 & Volts \\
Current in circuit $=$ & 0.000700882 & Amps \\
Initial resistance $=$ & 267.727587 & $\Omega$ \\
Final resistance $=$ & 560.5591504 & $\Omega$ \\
Sensitivity $=$ & 13.93440701 & $\Omega / \mu \mathrm{m}$ \\
\hline
\end{tabular}
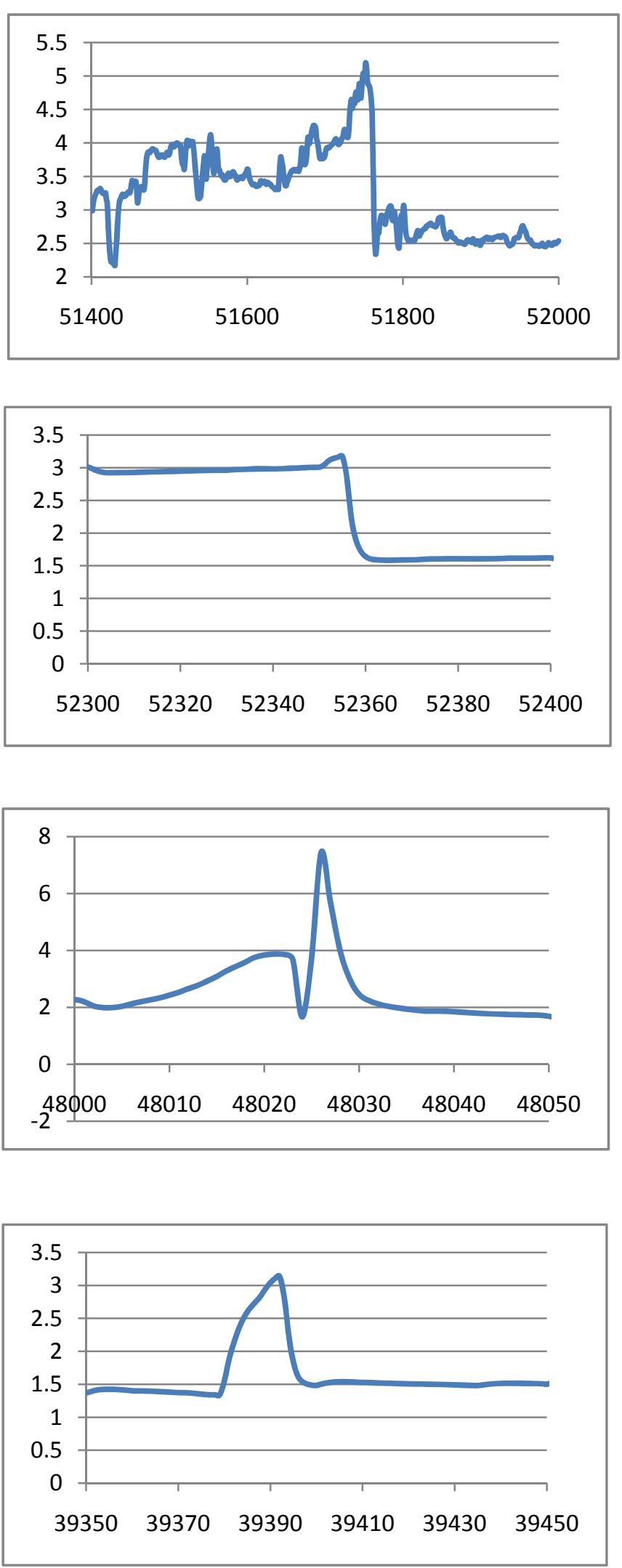
Device 7-30.2

\begin{tabular}{|ccc|}
\hline \multicolumn{3}{|c|}{ Beam 8} \\
\hline Maximum deflection & & \\
Max voltage output $=$ & 0.018279873 & Volts \\
Min voltage output $=$ & 0.002235949 & Volts \\
Current in circuit $=$ & 0.000714126 & Amps \\
Initial resistance $=$ & 3.131029196 & $\Omega$ \\
Final resistance $=$ & 25.59754627 & $\Omega$ \\
Sensitivity $=$ & 1.57898001 & $\Omega / \mu \mathrm{m}$ \\
\hline
\end{tabular}

Device 7-30.2

\begin{tabular}{|ccc|}
\hline \multicolumn{3}{|c|}{ Beam 9 } \\
\hline Maximum deflection & 13.81 & $\mu \mathrm{m}$ \\
Max voltage output $=$ & 0.010471772 & Volts \\
Min voltage output $=$ & 0.004311266 & Volts \\
Current in circuit $=$ & 0.000713978 & Amps \\
Initial resistance $=$ & 6.038375456 & $\Omega$ \\
Final resistance $=$ & 14.66680426 & $\Omega$ \\
Sensitivity $=$ & 0.624795714 & $\Omega / \mu \mathrm{m}$ \\
\hline
\end{tabular}

Device 8-30

\begin{tabular}{|ccc|}
\hline \multicolumn{3}{|c|}{ Beam 4} \\
\hline Maximum deflection & 12.53 & $\mu \mathrm{m}$ \\
Max voltage output $=$ & 0.001558801 & Volts \\
Min voltage output $=$ & 0.001092399 & Volts \\
Current in circuit $=$ & 0.000714208 & Amps \\
Initial resistance $=$ & 1.529525664 & $\Omega$ \\
Final resistance $=$ & 2.182559452 & $\Omega$ \\
Sensitivity $=$ & 0.052117621 & $\Omega / \mu \mathrm{m}$ \\
\hline
\end{tabular}

Device 8-30

\begin{tabular}{|ccc|}
\hline \multicolumn{3}{|c|}{ Beam 8} \\
\hline Maximum deflection & & \\
Max voltage output $=$ & 0.025198307 & Volts \\
Min voltage output $=$ & 0.004643123 & Volts \\
Current in circuit $=$ & 0.000713954 & Amps \\
Initial resistance $=$ & 6.503391906 & $\Omega$ \\
Final resistance $=$ & 35.29401749 & $\Omega$ \\
Sensitivity $=$ & 1.672852362 & $\Omega / \mu \mathrm{m}$ \\
\hline
\end{tabular}
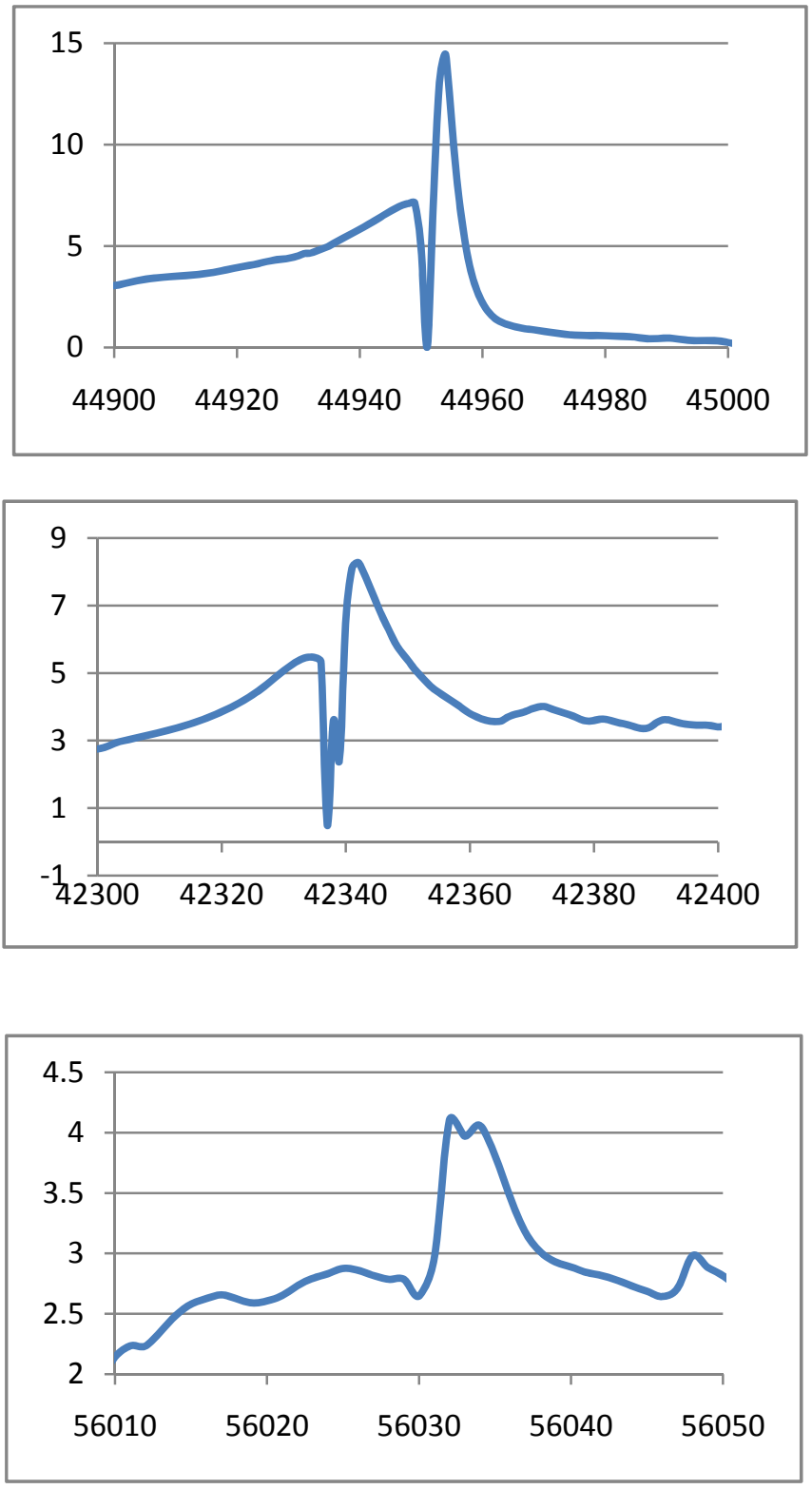


\section{LIST OF REFERENCES}

Abadal, G, et al. "Electromechanical model of a resonating nano-cantilever-based sensor for high-resolution and high-sensitivity mass detection." Institute of Physics Publishing, 2001: 100-104.

Abedinov, N, P Grabiec, T Gotszalk, T Ivanov, J Voigt, and I W Rangelow. "Micromachined piezoresistive cantilever array with integrated resistive microheater for calorimetry and mass detection." Journal of Vacuum Science \& Technology A: Vacuum, Surfaces, and Films 19, no. 6 (November 2001): 2884-2888.

Agoston, A, F Keplinger, and B Jakoby. "Evaluation of a vibrating micromachined cantilever sensor for measuring the viscosity of complex organic liquids." Sensors and Actuators A 82 (2005): 123.

Albrecht, T R, S Akamine, T E Carver, and C F Quate. "Microfabrication of cantilever styli for the atomic force microscope." Journal of Vacuum Science \& Technology A: Vacuum, Surfaces, and Films 8, no. 4 (July 1990): 3386-3396.

Alley, R L, G J Cuan, R T Howe, and K Komvopoulos. "The effect of release-etch processing on surface microstructurestiction." Solid-State Sensor and Actuator Workshop, 1992. 5th Technical Digest., IEEE. Hilton Head Island, 1992. 202-207.

Antonik, M D, N P DCosta, and J H Hoh. "A biosensor based on micromechanical interrogation of living cells." IEEE Eng. Med. Biol. 16 (1997): 66.

Bargatin, I., E. Myers, J. Arlett, B. Gudlewski, and M. Roukes. "Sensitive detection of nanomechanical motion using piezoresistive signal downmixing." Applied Physics Letters, 2005.

Baselt, D R, G U Lee, and R J Colton. "Biosensor based on force microscope technology." Jornal of Vacuum Science Technology 14 (1996): 789.

Battiston, F M, et al. "A chemical sensor based on a microfabricated cantilever array with simultaneous resonance-frequency and bending readout." Sensors and Actuators B, 2001: 122-131.

Blencowe, M P. "Nanoelectromechanical systems." Contemporary Physics 46 (2005): 249.

Bradshaw, R. D., P. C. Fletcher, Y. Xu, B. W. Alphenaar, and R. S. Keynton. "Model Optimization of a Piezoresistive Microactuation-Based Sensor For Gas Composition Analysis." ASME McMat. Austin, TX, 2007. 
Brugger, J, R A Buser, and N F de Rooij. "Micromachined atomic force I microprobe with integrated I." J. Micromech. Microeng. 2, 1992: 218-220.

Brysek, J, J R Mallon K Petersen, L Christel, and F Pourahmadi. "Silicon sensors and microstructures." Nava Sensor. Freemont, CA, 1991.

Butt, H J, M Jaschke, and W Ducker. "Measuring surface forces in aqueous-electrolyte solution with the atomic-force microscope." Bioelectrochem. Bioenerg. 38 (1995): 191.

Carlen, E T, and C H Mastrangelo. "Simple, high actuation power, thermally activated paraffin microactuator." Presented at the Transducers'99 Conference. Sendau, Japan, 1999.

Carr, D W, S Evoy, L Sekaric, H G Craighead, and J M Parpia. "Measurement of mechanical resonance and losses in nanometer silicon wires." Applied Physics Letters 75, no. 7 (August 1999): 920-922.

Chen, Y, and A Pepin. "Nanofabrication: conventional and nonconventional methods." Electrophoresis 22 (2001): 187.

Cherian, S, A Mehta, and T Thundat. "Investigating the mechanical effects of absorption of Ca2+ ions on a silicon nitride microcantilever surface." Langmuir, 2002: 6935.

Chopra, S, S Natarajan, and A M Rao. "Gas sensing using carbon nanotube-based resonator." Sensors, October 2004: 399-402.

Chu, Wen-Hwa, M. Mehregany, and R. Mullen. "Analysis of tip deflection and force of a bimetallic cantilever." Journal of Micromechanics and Microengineering, 1993: 4-7.

Chui, B W, T W Kenny, H J Mamin, B D Terris, and D Rugar. "Independent detection of vertical and lateral forces with a sidewallimplanted." Applied Physics Letters 72, no. 11 (March 1998): 1388-1390.

Craighead, H G. "Nanoelectromechanical Systems." Science 290 (2000): 1532.

Datskos, P G, et al. "Chemical detection based on adsorption-induced and photoinduced stresses in microelectromechanical systems devices." Journal of Vacuum Science \& Technology B: Microelectronics and Nanometer Structures 19, no. 4 (July 2001): 1173-1179.

Davila, A P, Jang,J, A K Gupta, T Walter, A Aronson, and R Bashir. "Microresonator mass sensors for detection of Bacillus anthracis Sterne spores in air and water." Biosensors and Bioelectronics 22, no. 12 (June 2007): 3028-3035. 
Ekinci, K L, X M H Huang, and M L Roukes. "Ultrasensitive nanoelectromechanical mass detection." Applied Physics Letters, 2003.

Elwenspoek, M, and H V Jansen. Silicon Micromachining. Cambridge University Press, 1999.

F. Blom, S. Bouwstra, M. Elwenspoek, and J. Fluitman. "Dependence of the quality factor or micromachined silicon beam resonators on pressure and geometry." Journal of Vacuum Science Technology B, 1992: 19-26.

Furukawa, T, K Ishida, and E Fukada. "Piezoelectric properties in the composite systems of polymers and PZT ceramics." Journal of Applied Physics 50, no. 7 (July 1979): 4904-4912.

Gaucher, P, D Eichner, J Hector, and W von Munch. "Piezoelectric bimorph cantilever for actuating and sensing applications." Journal of Physics 8 (1998): 235-238.

Gel, M, and I Shimoyama. "Force sensing submicrometer thick cantilevers with ultrathin piezoresistors by rapid thermal diffusion." Journal of Micromech. Microeng. 14 (2004): 423.

Grogan, C, et al. "Characterization of an antibody coated microcantilever as a potential immuno-based biosensor." Biosens. Bioelectron. 17 (2002): 201.

$\mathrm{Gu}, \mathrm{T}$, et al. "Impact of ploysilicon dry-etching on 0.5-MU-M NMOS transistor performance - the presence of both plasma bombardment damage and plasm charging damage." IEEE Electron Device Letters 15 (1994): 48.

Gupta, A, D Akin, and R Bashir. "Single virus particle mass detection using microresonators and nanoscale thickness." Applied Physics Letters 84, no. 11 (March 2004): 1976-1978.

Hirata, S, Y Ishii, H Matoba, and T Inui. "An ink-jet Head Using Diaphragm Microactuator." Micro Electro Mechanical Systems, 1996, MEMS '96, Proceedings. 'An Investigation of Micro Structures, Sensors, Actuators, Machines and Systems'. IEEE, The Ninth Annual International Workshop on. San Diego, CA, 1996. 418-423.

Ilic, B, D Czaplewski, H G Craighead, P Neuzil, C Campagnolo, and C Batt. "Mechanical resonant immunospecific biological detector." Applied Physics Letters 77 (2000): 450.

Jaeger, R C. Introduction to Microelectronic Fabrication: Volume 5 of Modular Series on Solid State Devices. 2nd Edition. New Jersey: Prentice Hall, 2002. 
Jafri, I H, H Busta, and S T Walsh. "Critical point drying and cleaning for MEMS technology." Proceedings of SPIE - MEMS Reliability for Critical and Space Applications. 1999. 51-58.

Ji, H F, E Finot, R Dabestani, T Thundat, G M Brown, and P F Britt. "A novel selfassembled monolayer (SAM) coated microcantilever for low level cesium detection." Chem. Commun. 6 (2000): 457.

Ji, H F, K M Hansen, Z Hu, and T Thundat. "Detction of $\mathrm{pH}$ variation using modified microcantilever sensors." Sensors and Actuators B 72 (2001): 233.

Ji, H F, T Thundat, R Dabestani, G M Brown, P F Britt, and P V Bonnesen. "Ultrasensitive detection of CrO42- using a microcantilever sensor." Aanl. Chem. 73 (2001): 1572.

Judy, J W. "Microelectromechanical systems (MEMS): fabrication, design, and applications." Smart Mater. Struc. 10 (2001): 1115.

Judy, J W. "Microelectromechanical systems (MEMS): fabrication, design, and applications." Smart Mater. Struct. 10 (2001): 1115-1134.

Kenny, T. "Nanometer-scale force sensing with MEMS devices." Sensors Journal 1, no. 2 (August 2001): 148-157.

Kim, K H, J S Ko, Y-H Cho, K Lee, B M Kwak, and K Park. "A skew-symmetric cantilever accelerometer for automotive airbag applications." Sensors and Actuators A: Physical 50, no. 1 (August 1995): 121-126.

Klaassen, E H, et al. "Silicon Fusion Bonding and Deep Reactive Ion Etching a New Technology for Microstructures." Solid-State Sensors and Actuators, 1995 and Eurosensors IX. Transducers '95. Stockholm, Sweden, 1995. 556-559.

Kooser, A., K. Manygoats, M. Eastman, and T. Porter. "Investigation of the antigen anitbody reaction between anti-bovine serum albumin (a-BSA) and bovine serum albumin (BSA) using piezoresistive microcantilever based sensors." Biosensors and Bioelectronics, 2003: 503-508.

Laermer, F, and A Urban. "Challenges, developments and applications of silicon deep reactive ion etching." Microelectronic Engineering, June 2003: 349-355.

Laermer, F., and A. Schilp. Method of anisotropically etching silicon. United States Patent 5501893. March 26, 1996.

Lang, H P, et al. "A chemical sensor based on a micromechanical cantilever array for the identification of gases and vapors." Applied Physics A: Materials Science \& Processing 66 (March 1998). 
Lang, H P, et al. "An artificial nose based on a micromechanical cantilever array." Analytica Chimica Acta 393 (1999): 59-65.

Lang, H P, M Hegner, and C Gerber. "Cantilever array sensors." Materials Today, April 2005: 30-36.

Lange, D, et al. IEEE international conference on micro electro mechanical systems, technical digest. Orlando, 1999. 447.

Lavrik, N V, M J Sepaniak, and P G Datskos. "Cantilever transducers as a platform for chemical and biological sensors." Review of Scientific Instruments 75, no. 7 (July 2004): 2229-2253.

Lee, Caroline, H Nam, Y Kim, W Jin, S Cho, and J Bu. "Microcantilevers integrated with heaters and piezoelectric detectors." Applied Physics Letters 83, no. 23 (December 2003): 4839-4841.

Lee, Seung, and R. White. "Self-excited piezoelectric cantilever oscillators." Sensors and Actuators A: Physical, 1996: 41-45.

Li, Xinxin, Takahito Ono, Yuelin Wang, and Masayoshi Esashi. "Ultrathin singlecrystalline-silicon cantilever resonators: Fabrication technology and significant specimen size effect on Young's modulus." Applied Physics Letters 83, no. 15 (October 2003): 3081-3083.

Madou, M J. Fundamentals of Microfabrication. CRC Press, 1997.

Maluf, N. An Introduction to Microelectromechanical Systems Engineering. Artech House, 2000.

Mamin, H J, and D Rugar. "Sub-attonewton force detection at millikelvin temperatures." Applied Physics Letters 79 (2001): 3358.

Marty, F, et al. "Advanced etching of silicon based on deep reactive ion etching for silicon high aspect ratio microstructures and three-dimensional micro- and nanostructures." Microelectronics Journal 36, no. 7 (July 2005): 673-677.

Mei, T, W J Li, Y Ge, Y Chen, L Ni, and M H Chan. "An integrated MEMS threedimensional tactile sensor with large force." Sensors and Actuators 80 (2000): 155162.

Meyer, G, and N M Amer. "Novel optical approach to atomic force microscopy." Applied Physics Letters 53 (1988): 1045. 
Morante, M, A Cobo, and J M Lopezhiquera. "New approach using a bare fiber optic cantilever beam as a low-frequency acceleration measuring element." Optical Engineering 35 (1996): 1700.

Muller, P, and R Kern. "About the measurement of absolute isotropic surface stress of crystals." Surf. Sci. 301 (2001): 386-398.

Murad, S, M Rahman, N Johnson, S Thomas, S P Beaumont, and C D Wilkinson. "Dry etching damage in III-V semiconductors." Journal of Vacuum Science Technology 14 (1996): 3658.

Pan, Chi Shiang, and Wensyang Hsu. "An electro-thermally and laterally driven polysilicon microactuator." Journal of Micromech. Microeng. 7 (1997): 7-13.

Partridge, A, et al. "A high-performance planar piezoresistive accelerometer." Microelectromechanical Systems 9, no. 1 (March 2000): 58-66.

Porter, T L, M P Eastman, D L Pace, and M Bradley. "Sensor based on piezoresistive microcantilever technology." Sensors and Actuators A: Physical 88, no. 1 (January 2001): 47-51.

Preissig, F J von. "Applicability of the classical curvature-stress relation for thin films on plate substrates." Applied Physics 66 (2001): 4262-4268.

Riehl, P S, K L Scott, R S Muller, R T Howe, and J A Yasaitis. "Electrostatic charge and field sensors based on micromechanical resonators." Microelectromechanical Systems 12, no. 5 (October 2003): 577-589.

Roukes, M L. "Nanoelectromechanical Systems Face the Future." Physics World 14 (2001): 25 .

Salzberg, A D, M B Bloom, N J Mourlas, and T M Krummel. "Microelectrical mechanical systems in surgery and medicine." Journal of the American College of Surgeons 194, no. 4 (April 2002): 463-476.

Scuor, N, P Gallina, O Sbaizero, and H V Panchawagh. "Dynamic characterization of MEMS cantilevers in liquid environment using a low-cost optical system." Meas. Sci. Technol. 17 (2006): 173-180.

Stowe, T D, K Yasumura, T W Kenny, D Botkin, K Wago, and D Rugar. "Attonewton force detection using ultrathin silicon cantilevers." Applied Physics Letters 71 (1997): 288.

$\mathrm{Su}$, Ming, Shuyou Li, and Vinayak Dravid. "Microcantilever resonance-based DNA detection with nanoparticle probes." Applied Physics Letters 82, no. 20 (May 2003): 3562-2564. 
Su, Y, AG R Evans, A Brunnschweiler, and G Ensell. "Charaterization of a highly sensitive ultra-thin piezoresistive silicon cantilever probe and its application in gas flow velocity sensing." J. of Micromechanics and Microengineering 12 (2002): 780.

Sze, S M. Semiconductor Devices: Physics and Technology. New York: John Wiley, 1985.

Thaysen, J, A Boisen, O Hansen, and S Bouwstra. "Atomic force microscopy probe with piezoresistive read-out and a highly symmetrical wheatstone bridge arrangement." Sensors and Actuators A 83 (1999): 47.

Thiele, J A, and M P da Cunha. "High temperature SAW gas sensor on langasite." Sensors 2 (October 2003): 769-772.

Thundat, T, E A Wachter, S L Sharp, and R J Warmack. "Detection of mercury vapor using resonating microcantilevers." Applied Physics Letters 66 (1995): 1695.

Thundat, T, G Y Chen, R J Warmack, and E A Wachter. "Vapor detection using resonating microcantilevers." Analytical Chemistry 67 (1995): 519.

Tipple, C A, N V Lavrik, M Culha, J Headrick, P Datskos, and M J Sepaniak. "Nanostructured microcantilevers with functionalized cyclodextrin receptor phases: self-assembled monolayers and vapor-deposited films." Anal. Chem. 74 (2002): 3118.

Tortonese, M, R C Barrett, and C F Quate. "Atomic force microscope using piezoresistive cantilever." IEEE Proceedings of Transducers '91. New York, 1991. 448.

Tortonese, M, R C Barrett, and C F Quate. "Atomic resolution with an atomic force microscope using piezoresistive detection." Applied Physics Letters 62 (1993): 834.

Tufte, O N, and E L Stelzer. "Piezoresistive properties of silicon diffused layers." Applied Physics Letters 34 (1963): 313.

Volklein, F, M Blumers, and L Schmitt. " Thermoelectric microsensors and microactuators (MEMS) fabricated bythin film technology and micromachining." Thermoelectrics, 1999: 285-293.

Wang, L, et al. "Design, Fabrication, and Measurement of High-Sensitivity Piezoelectric Microelectromechanical Systems Accelerometers." Journal of Microelectromechanical Systems 12, no. 4 (August 2003): 433-439.

Wang, L, Y Zhao, E Ng, and Q Lin. " A MEMS differential calorimeter for biomolecular characterization." Micro Electro Mechanical Systems, 2005: 814-817. 
Willemin, M, et al. "Piezoresistive cantilever designed for torque magnetometry." Journal of Applied Physics 83, no. 3 (February 1998): 1163 - 1170.

$\mathrm{Xu}, \mathrm{T}, \mathrm{G} \mathrm{Wu}, \mathrm{G}$ Zhang, and Y Hao. "The compatibility of $\mathrm{ZnO}$ piezoelectric film with micromachining process." Sensors and Actuators A: Physical 104, no. 1 (2003): 6167.

Xu, Yang. "Fabrication, Development, and Characterization of Actuation Based Sensors and Applications for Gas Analysis." Doctorate of Philosophy Dissertation. University of Louisville, 2006.

Xu, Yang, J T Lin, B W Alphenaar, and R S Keynton. "Viscous damping of microresonators for gas composition analysis." Applied Physics Letters 88 (April 2006): 143513.

Yao, J J. "RF MEMS from a device perspective." J. Micromech. Microeng. 10 (2000): R9-R38.

Yasuda, T, I Shimoyama, and H Miura. " CMOS drivable electrostatic microactuator with large deflection." Micro Electro Mechanical Systems, 1997. MEMS '97, Proceedings, IEEE., Tenth Annual International Workshop on. Nagoya, Japan, 1997. 90-95.

Yuan, C W, E Batalla, M Zacher, A L de Lozanne, M D Kirk, and M Tortonese. "Low temperature magnetic force microscope utilizing a piezoresistive cantilever." Applied Physics Letters 65, no. 10 (September 1994): 1308-1310.

Ziegler, Christiane. "Cantilever-based biosensors." Anal Bioanal Chem 379 (2004): 946959.

Zribi, A, A Knobloch, and R Rao. "CO2 detection using carbon carbon nanotube networks and micromachined resonant transducers." Applied Physics Letters 86 (May 2005): 203112. 


\section{VITA}

PATRICK CARL FLETCHER

3128 Commander Drive, Louisville, KY 40220

502.296.8208 (cell)

patrick.c.fletcher@gmail.com

\section{EDUCATION}

University of Louisville, Louisville, $K Y$

Master of Engineering with Highest Honors in Mechanical Engineering

Thesis: "Alternative Piezoresistor Designs for Maximizing Cantilever Sensitivity"

Honors: Selected as Speed School Outstanding Student to represent engineering

school at commencement ceremony by leading graduating class

University of Louisville, Louisville, $K Y$

M.B.A. in Business Administration

University of Louisville, Louisville, $K Y$

B.S. With Highest Honors in Mechanical Engineering

Honors Thesis: "Implementation of Pivot-Plate Resonators (PPR) in the Detection

of Nanogram Quantities on Mass"

\section{AWARDS}

- J.H. Hilliard Scholarship

- Lewis. S. Streng Award

- ASHRAE Outstanding Student Award

- Alfred T. Chen Memorial Scholarship Award

- F.W. "Beich" Beichley Scholarship

$2005-2006$

- Provost Hallmark Award

$2002-2007$

- KEES Scholarship

$2002-2006$

- Speed School Alumni Scholarship

2002

\section{TEACHING EXPERIENCE}

University of Louisville, Louisville, $K Y$

Supplemental Instructor - in "Differential Equations"

Held 3 classes per week, prepared practice exams and problems, supplemented the EAC 205 curriculum for students needing additional instruction.

Supplemental Instructor - in "Engineering Analysis Core 101"

Held 3 classes per week, prepared practice exams and problems, supplemented the EAC 101 curriculum for students needing additional instruction.

\section{RELATED EXPERIENCE}

University of Louisville, Louisville, $K Y$

Graduate Research Assistant

Designed, simulated, fabricated, and tested MEMS devices in a class 100/1000 cleanroom. Performed 3-D modeling and analysis of MEMS devices and collected data using data acquisition systems. Prepared journal and conference papers, as well as posters for research presentations. 
Sandia National Laboratories, Albuquerque, NM

Student Intern

Performed 3 required co-ops for the Micro-Total-Analytical Systems

department as a student. Obtained an " $\mathrm{L}$ " security clearance for working on

confidential projects. Job duties included 3-D modeling and analysis, computer

programming, circuit design \& wiring, and metal \& polymer part fabrication.

\section{PUbliCATIONS AND PAPERS}

- "Alternative Piezoresistor Designs for Maximizing Microcantilever Sensitivity" Masters Thesis in mechanical engineering, Louisville, KY 2008

- "Piezoresistive Geometry for Maximizing Microcantilever Array Sensitivity" Lead author on paper presented at IEEE Sensors, Lecce, Italy 2008

- "Piezoresistive Geometry for Maximizing Microcantilever Array Sensitivity" Lead author on poster presented at UGIM, Louisville, KY 2008

- "Model Optimization of a Piezoresistive Microactuation-Based Sensor for Gas Composition Analysis"

Contributing author on paper presented at the ASME McMat, Austin, TX 2007

\section{LANGUAGES}

- English - native language

- German - conversationally fluently

\section{MEMBERSHIPS}

- American Society of Mechanical Engineers (ASME)

- American Association for the Advancement of Science (AAAS) 